\title{
An Automatic Verification Tool for Firewall Configurations
}

By

Feng Zheng

\author{
A thesis submitted to \\ The Faculty of Graduate Studies and Research \\ In partial fulfilment of \\ The requirements for the degree of \\ Master of Science \\ Information and Systems Science
Ottawa-Carleton Institute for Computer Science
School of Computer Science
Carleton University
Ottawa, Ontario

August, 2006

(C) Copyright

2006, Feng Zheng 


$\begin{array}{ll}\begin{array}{l}\text { Library and } \\ \text { Archives Canada }\end{array} & \begin{array}{l}\text { Bibliothèque et } \\ \text { Archives Canada }\end{array} \\ \begin{array}{l}\text { Published Heritage } \\ \text { Branch }\end{array} & \begin{array}{l}\text { Direction du } \\ \text { Patrimoine de l'édition }\end{array} \\ \begin{array}{l}\text { 395 Wellington Street } \\ \text { Ottawa ON K1A ON4 }\end{array} & \begin{array}{l}\text { 395, rue Wellington } \\ \text { Ottawa ON K1A ON4 } \\ \text { Canada }\end{array}\end{array}$

Your file Votre référence ISBN: 978-0-494-18381-6 Our file Notre référence ISBN: 978-0-494-18381-6

NOTICE:

The author has granted a nonexclusive license allowing Library and Archives Canada to reproduce, publish, archive, preserve, conserve, communicate to the public by telecommunication or on the Internet, loan, distribute and sell theses worldwide, for commercial or noncommercial purposes, in microform, paper, electronic and/or any other formats.

The author retains copyright ownership and moral rights in this thesis. Neither the thesis nor substantial extracts from it may be printed or otherwise reproduced without the author's permission.
AVIS:

L'auteur a accordé une licence non exclusive permettant à la Bibliothèque et Archives Canada de reproduire, publier, archiver, sauvegarder, conserver, transmettre au public par télécommunication ou par l'Internet, prêter, distribuer et vendre des thèses partout dans le monde, à des fins commerciales ou autres, sur support microforme, papier, électronique et/ou autres formats.

L'auteur conserve la propriété du droit d'auteur et des droits moraux qui protège cette thèse. $\mathrm{Ni}$ la thèse ni des extraits substantiels de celle-ci ne doivent être imprimés ou autrement reproduits sans son autorisation.
In compliance with the Canadian

Privacy Act some supporting forms may have been removed from this thesis.

While these forms may be included in the document page count, their removal does not represent any loss of content from the thesis.
Conformément à la loi canadienne sur la protection de la vie privée, quelques formulaires secondaires ont été enlevés de cette thèse.

Bien que ces formulaires aient inclus dans la pagination, il n'y aura aucun contenu manquant.

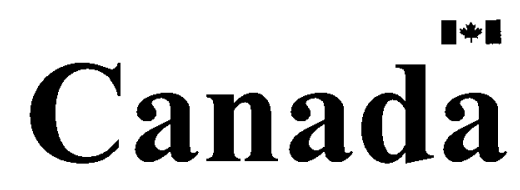




\begin{abstract}
Firewalls are essential to information security. However, it can be very difficult to configure firewalls correctly to realize a security policy. The more complicated firewalls are, the easier it is to make a mistake and the more dangerous it is. Errors of firewall configurations are a chief factor in firewall failure.

Most traditional tools for analyzing firewall configurations, based on traditional testing methods, are unreliable and incomplete. Attackers may only need to exploit a single vulnerability to cause great damage. Reliable and complete tools that can be used before firewall deployment [11] are important, but only a small number of such tools have been built, and they all have serious limitations.

This thesis shows how a tool overcoming these limitations can be developed easily by borrowing tools and ideas from the area of computer-aided formal verification. The tool is implemented using the SMV language and system.
\end{abstract}




\section{Acknowledgements}

First and foremost I would like to thank Dr. Douglas Howe for his constant support and encouragement throughout my graduate studies. His ideas and insights are essential to the completion of this thesis.

I would also like to thank Dr. Anil Somayaji and the system administrator of the school for providing valuable data during my test.

I am grateful to my fellow graduates, the faculty and staff of the school of computer science and mathematics for the friendship and help. I would like to thank them for widening my mind and knowledge.

Last, I would like to thank my family for their support and love. 


\section{TABLE OF CONTENTS}

CHAPTER 1: Introduction

$1.1 \quad$ Firewalls.................................................................. 1

1.2 A graph model of firewalls ............................................ 3

1.3 Challenging problems of firewall configuration........................... 8

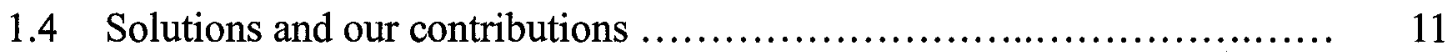

CHAPTER 2: Previous solutions .............................. 17

2.1 FANG : A firewall analysis engine, a graph approach................... 17

2.1.1 The basic graph model .................................... 17

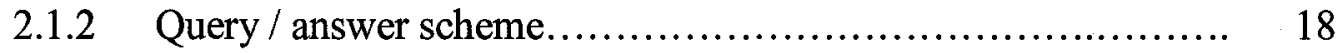

2.1.3 Outline of FANG implementation........................... 22

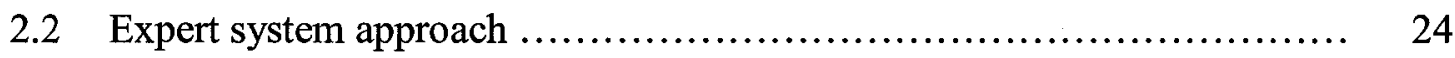

2.2.1 Introduction ................................................. 24

2.2.2 Implementation of an expert system for firewall configuration $\ldots \quad 24$

2.2.3 User interface and example.................................. 27

$2.3 \quad$ Boolean expression approach............................................ $\quad 30$

2.3.1 Introduction............................................... 30

2.3.2 Ordered Binary Decision Diagram (OBDD) ................... 30

2.3.3 Algorithm for analyzing a rule set ............................ 34

2.3.4 Examples ................................................. 38

2.4 FIREMAN............................................................. 42

iv 
2.4.1 Introduction................................................ 42

2.4.2 Misconfigurations ............................................. 30

2.4.3 Comparison with our approach .................................. 34

CHAPTER 3: Philosophy of verification approach for multi-firewall configurations..................................

CHAPTER 4: Model data flow over the internet using SMV ...... 53

$4.1 \quad$ Data structure.................................................. 58

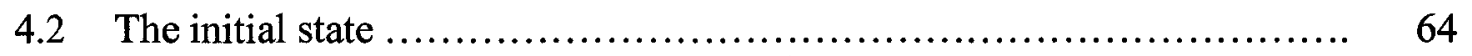

4.3 The next state.................................................. 67

CHAPTER 5: How to answer the challenging questions of firewall 75

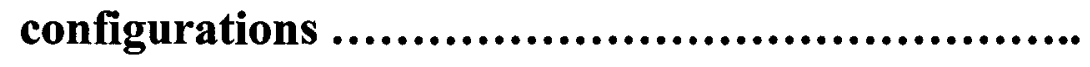

CHAPTER 6: Performance of our approach................... $\quad 80$

CHAPTER 7: Conclusions and future works....................... 85

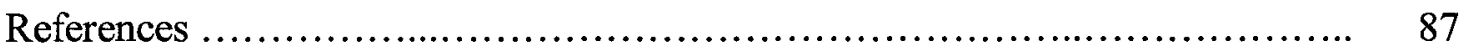

Appendices ........................................................ 89 


\section{LIST OF FIGURES}

Figure $1.1 \quad$ Graph at a simple firewall ....................................... 4

Figure 1.2 A novel multi firewall configuration for a corporation networks 6

Figure 1.3 A graph model for firewalls for the corporation networks ......... 7

Figure 2.1 A corporation networks for multi-firewalls ...................... 19

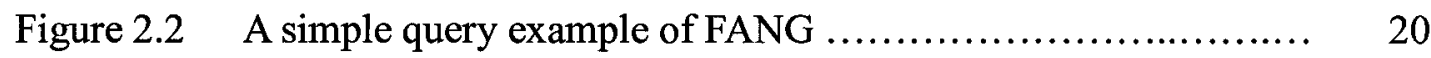

Figure $2.3 \quad$ Another query example of FANG …....................... 21

Figure 2.4 A spoofing example of FANG............................... 21

Figure 2.5 FANG architecture and data flow ............................. 22

Figure 2.6 Packet representation in the system using Eclipse.................. 25

Figure 2.7 Match list predicate in the expert system .......................... 26

Figure $2.8 \quad$ The network of a small company.............................. 27

Figure 2.9 An example of command list in the expert system using Eclipse .. 28

Figure 2.10 An query example using the expert system ...................... 28

Figure 2.11 A truth table of boolean function................................ 31

Figure 2.12 A binary decision tree of boolean function ...................... 31

Figure 2.13 A Binary Decision Diagram (BDD) of boolean function .......... 33

Figure 2.14 Tabular form of results of a query of a firewall rule set .......... 40

Figure 2.15 Query of consequences with firewall rule change ................. 41

Figure 2.16 Firewall files and misconfigurations................................ 43

Figure 3.1 A state transition system generated by a SMV program ............ 50

Figure 4.1 A simple example of multi personal firewalls....................... 53

Figure 6.1 An example of multi-firewall configurations of an institution ...... 81 


\section{LIST OF APPENDICES}

Appendix 1: Code for the example of multiple personal firewall ............... 89

Appendix 2: The core code of our tool .................................. 93

Appendix 3: Code for the example of multiple firewalls for an institution ....... 99

vii 


\section{Chapter 1}

\section{Introduction}

\subsection{Firewalls}

Modern society is heavily dependent on the information infrastructure. For example there would be no modern banking without computers. This is true partly due to the powerful information processing capability of computers. However, computers are only isolated information islands without connected networks. It is the networks that enable a modern bank to run its transactions so productively and interactively. More generally, the Internet has brought profound benefits and has had a critical impact on society.

Unfortunately, connected networks bring not only benefits but also threats. For instance, Air Canada had filed a lawsuit of millions dollars against a VP of West Jet because he illegally obtained seat reservation information from Air Canada via the Internet and therefore unfairly gained competitive advantage. Before the Internet, computer viruses spread mainly through floppy disks. Now computer viruses are spreading mainly through networks. This allows viruses to spread much more quickly and widely, making them much more serious threats. For example, in 2003 a computer virus spread via an email system disrupted Air Canada service half a day and stopped planes from flying. While an enterprise can gain tremendous advantages through networks, its business secrecy can also 
be stolen remotely and silently.

Losses caused by threats may sometimes outbalance gains. In reality information security has increasingly become a major hurdle for further and deeper application of information technology. Therefore how to curb threats from networks is a critically important task.

Clearly no harm can come to your networked computer if it becomes inaccessible to attacking computers or data packets from them. Any threats, including spying, must come via some network connection of your computer to another. You can reduce threats, sometime greatly, if you can restrict how other computers access your computers. This basic idea has fostered the fast development and application of firewall technology.

For an organization, it is critical to control information access. It is dangerous to permit data to go into and out of its networks without any restriction. So it is desirable to have a boundary, i.e. a firewall, to separate its networks from outside networks, such as the Internet. With a firewall, only permissible data can flow in and out; other data is blocked. This kind of limited accessibility can diminish possible threats, sometimes greatly, because some kinds of attacking data can be filtered out and kept out of touch of its computers and their services. A single firewall for an organization may not be enough. For example, a company may have requirements or demands, such as that the public can access some of its information from its web servers, but some confidential information is only shared within the organization. For this purpose at least three levels of access controls are needed.

At least two firewalls are usually needed to separate the whole network into three zones: the public Internet (outside networks), DMZ (De-Militarized Zone) for hosts such as web servers that may be accessible from the Internet, and internal networks that are only 
accessible to privileged users like employees. A data packet from one zone to another zone can pass or be dropped by relevant firewalls. The firewalls can block data packets from outside from reaching the internal servers and therefore eliminate potential attacks to them from the Internet. So a certain degree of security is achieved.

Three zones or a similarly simple form of network topology may be still too simple to satisfy needs of information access and security, even for a medium sized corporation. The zones define multiple levels of accessibility to services on computers of the company and therefore enforce or balance information accessibility and security.

In practice, the firewall is a basic and fundamentally effective means to protect information systems. Firewall technology has made great progress since its inception and been quite successful. However, how to configure firewalls correctly remains a crucial and challenging problem that has been lagging behind research and practice [11], and is the topic of the thesis. Before we study the problem in detail, let us look at the basic model of firewalls.

\subsection{A graph model of firewalls}

The Internet enables computers in different networks to talk with each other by conveying data packets, which start from a source computer and end at a destination one. During the communication process, data packets are continually forwarded to a next hop, or next network, usually by a router or gateway. From this respect the Internet, or any other network, can be regarded as a data packet flow graph. Without firewalls, this graph can be regarded as a single access zone, where computers can exchange data freely. A company 
usually uses a gateway, a dedicated computer or a router, to connect its networks to outside networks. Inside the company it may use the same technology to connect different parts of its different networks. Because this free exchange of information can cause threats, it is desirable to block unwanted data. A firewall usually sits in the gateway to fulfill the purpose. The firewall becomes a border gate between its networks and the Internet to manage data flow and restrict dangerous free exchange. The following Figure 1.1 shows this idea.

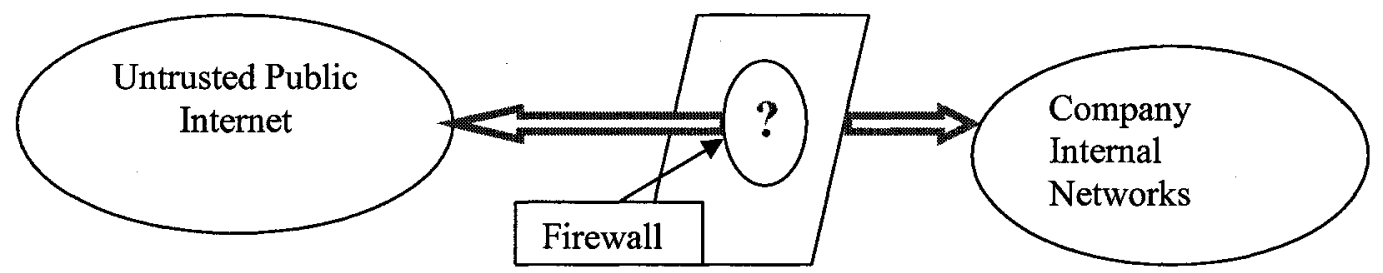

Figure 1.1 Graph of a simple firewall

The firewall limits the accessibility of computers in the internal networks from the Internet by blocking unwanted data through the firewall. As an extreme case, if the implemented firewall allows all data to pass in and out, it is equivalent to having no firewall in place. If it disallows all data in and out, it is equivalent to physically disconnecting the internal networks from the Internet and the internal networks become isolated.

There are many techniques for implementing a firewall. The basic one, which this thesis focuses on, is called the packet-filtering technique. A data packet is actually called an Internet Protocol (IP) datagram. We assume that every computer on a network has an 
unique IP address. Packets contain port numbers representing different services provided by a computer. Every packet has a header that consists of a source and destination IP address, a combination of protocols (TCP/UDP/...) and port numbers (source and destination) representing services, and so on. This is similar to an ordinary mail system, where mail has sender's and receiver's addresses, apartment numbers, etc. on the envelope. Based on the packet information in the header, a set of rules decides whether to pass or drop the packet when it goes through firewalls during its journey.

A typical rule consists of two parts:

- Condition: a matching criterion to match a set of packets.

- Action: pass or drop (actual firewall rules may have more actions, but they are out of the scope of the thesis).

If a packet fits in the match criterion or matching set, then the firewall takes the specified action of permitting or denying.

As an example: a rule in an access list for a Cisco firewall or router may be [7]:

Access-list 101 permit tcp 20.9.17.8 0.0.0.0 121.11.127.20 0.0.0.0 range 2327

The access list 101 means that any TCP packets from IP address 20.9.17.8 to 121.11.127.20 with port numbers between 23 and 27 are permitted or accepted. More detail on rules will be given later.

A firewall contains a set of order sensitive rules. If a specific data packet does not match a rule, the next rule in the rule list is tried. If the packet matches it, the action specified by the rule is immediately taken. Thus, if there are several rules satisfied for a specific packet, the first rule's action (pass or drop) is taken. These are also some firewall products that can specify to take the last rule's action, or a mixed order. However, there is 
no difference in principle, so we assume in the thesis that the first matching rule is always applied. The order of a rule set is certainly critical. A change of order of rules may result in permit or denial action switch for some packets, and cause different and unexpected consequences.

One firewall is often too simple for realizing security policy for an organization, and several firewalls are often needed. However, the functionality of packet filter rules of firewalls stays the same. Let us look at a multi firewall example from Figure 1.2 [11]:

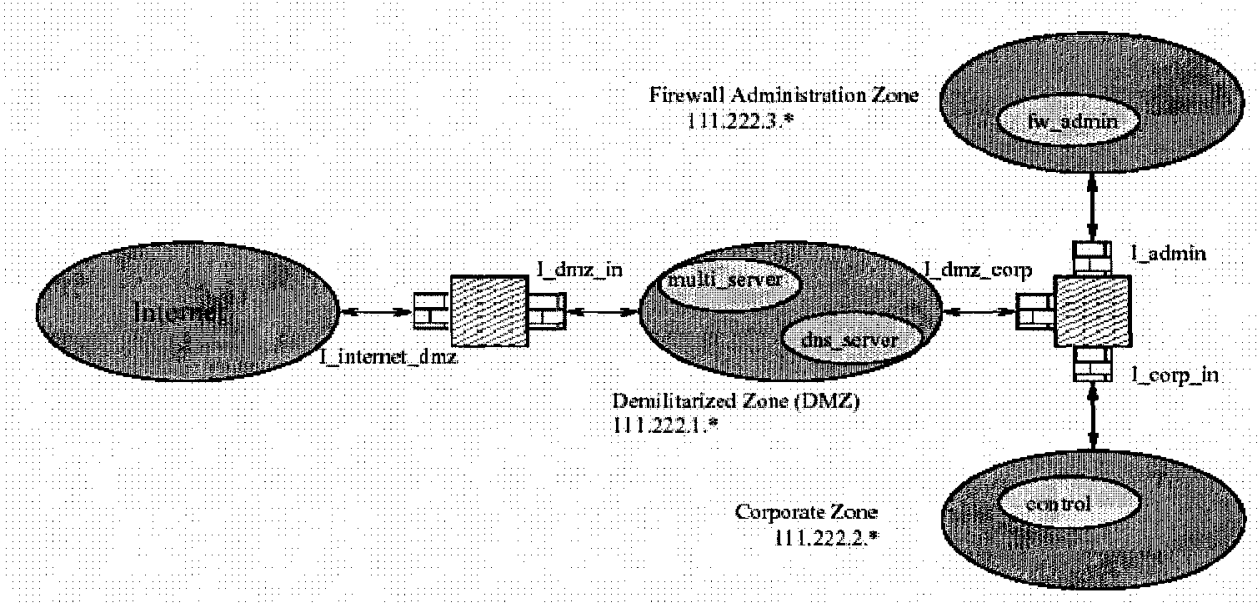

Figure 1.2 A novel multi firewall configuration for a corporation networks

The whole Internet is regarded as a 4-byte IP address space and every participating host is distinguished by a unique IP address. A computer may provide many services represented by protocol and port numbers. The above example divides the whole Internet IP address space into four disjoint zones of IP addresses (two gateways and five interfaces may have their own IP addresses that are not included in the zones) from the view of the corporate firewall administrator. The corporate intranet, or internal network, has three restricted zones. Five firewalls are deployed on the five interfaces attached on two 
gateways. A specific data packet can flow freely inside zones and is passed or dropped by relevant firewalls during its journey from its source address to its destination address when it goes among zones. We can naturally model data packet flow from one zone to another as a graph, as follows:

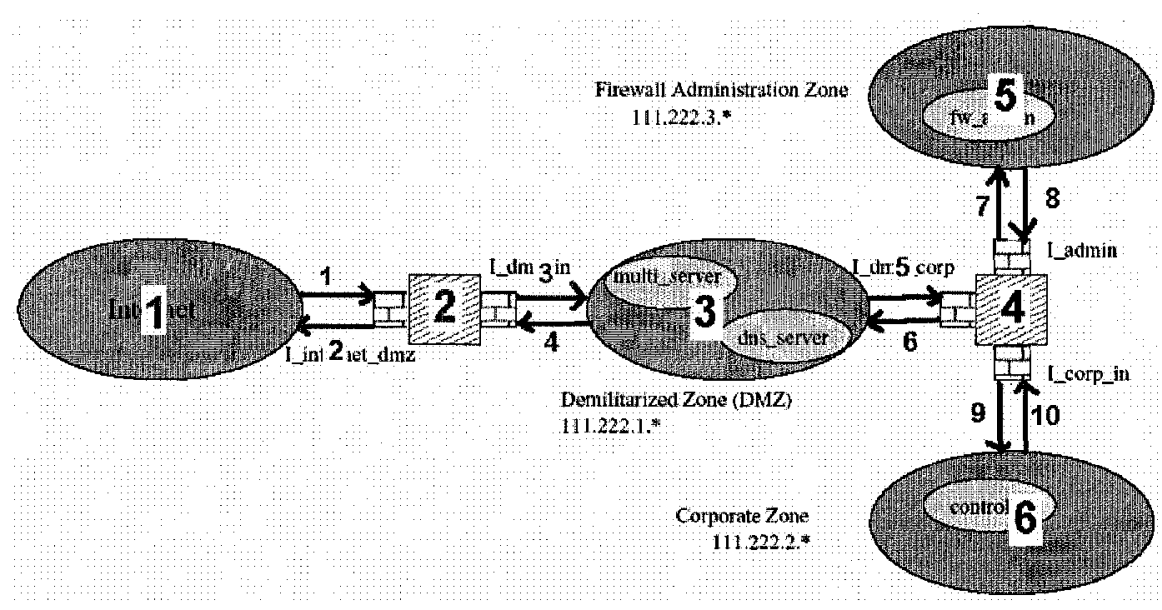

Figure 1.3 A graph model for firewalls for the corporation

In the graph model, a directed graph represents the network. A vertex represents a zone of computers, namely their IP addresses with port numbers; and a directed edge attaches a firewall rule set to restrict data flow between incidental vertices or nodes. Packets are assumed to travel freely inside a zone. There are 6 zones and 10 firewall rule sets, in which a single rule set manages one direction of data flow. The directed graph model demonstrates the core concept of multi firewall configuration.

In summary, we can model the Internet traffic with firewalls as a packet flow over a directed graph like the above. Because the goal of firewalls is to restrict packet flow across the zones, any security policy enforced by firewalls will ultimately be judged by:

1. What kinds of data packets should reach their destinations (zones) in order to 
preserve needed services.

2. What kinds of packets should not reach what zones in order to prevent potential attacking or sniffing data.

However, these goals are very hard to achieve with currently available tools. Since a packet includes source IP address and port number, destination IP address and port number, the space of possible packets is huge. The packet space alone may be bigger, more than $2^{100}$. So there arise some challenging problems in complex enterprise applications when setting up appropriate low-level firewall rules to correctly realize specified security policy of an organization.

\subsection{Challenging problems of firewall configuration}

Once firewalls are installed, it is crucial to correctly configure or set up low-level firewall rules to realize a security policy that is usually described in a high-level language. For example, a company may claim that the public can only access its web server services and not other services or servers. Then system administrators implement some rules, such as blocking any packets with destination addresses being those internal servers. This is easy in a simple case. However it can become very challenging when systems, security policies and software services are complicated. Rubin [13] claimed, "The single most important factor of your firewall's security is how you configure it".

Configuration is an important task because what kind of packets can pass or drop may directly link to security problems. It can also be a very difficult task. The difficulty comes 
from the fact that one has to use low-level languages to construct sets of rules to implement the high-level security policy accurately, while maintaining service and good performance. This is especially a problem in the complicated topology of networks of big institutions. In practice there may be hundreds of rules in a firewall.

In practice, it is hard to avoid mistakes in configuration. For an attacker, there may only need to be a single vulnerability to be exploited to launch an attack. However, a defender has to make sure that every implementation is correct from all aspects since even a minor mistake could open a door to possible attacks. With more services to meet increasing business demands, it is easier to make a mistake and potential damage may be very high. If too much is blocked, benefits provided by needed services may be lost.

The difficulty of the task is also due to the fact that understanding the consequences of a configuration is a daunting job, especially when there are hundreds of rules in sophisticated environments. Firewall configurations also need to constantly evolve with changing demands from development of applications, bugs being found, or a new person taking charge of security administration. The understanding or verification of firewall configurations is a first step to correctly implement a firewall configuration.

In order to understand firewall configurations, namely what kinds of packets will pass or be dropped by firewalls and their security meaning, and to further explain their security consequences, firewall administrators often need to answer some potentially difficult questions in a timely manner such as the following [7][6][11]:

1. "Can I telnet from here to there?"

2. "From which machines can our DMZ be reached, at what services?"

3. "What connections are allowed from host X to our intranet?" 
4. "What will be the effect adding this rule on this firewall"

These questions are specific, detailed and focused on general questions of what data will succeed or fail to reach their destination or certain zones. In simple cases, these questions may be easy to answer. However, especially in complicated corporate networks, there are some good reasons why these questions are very difficult, elusive and time-consuming [11] [7] [6]:

1. While understanding a firewall with a few rules is easy, it can be very difficult when there are many rules and their meaning is order sensitive. The overlapping packet matching sets represented by rules often increase the difficulty of understanding.

2. Packets have several paths from source to destination and may experience several firewalls.

3. Different firewalls of an organization may use different vendor products that have different configuration languages.

4. You cannot always infer global behaviors if you only examine firewalls separately.

5. Due to the large packet space, it is very difficult to achieve or verify the goal that all wanted packets pass and all unwanted packets are dropped by firewalls. Given the complexity in requirements and services of a corporate network, it is challenging to say with confidence what packets can travel or not under firewall restrictions. If you let unwanted packets pass, your networks are open to attack. If you block wanted packets, you may disrupt needed services.

There is one particular reason above all those mentioned: inadequate tools to support answering the kinds of challenging questions given above for firewall security policy. Until 
recently there has been little progress on this topic compared to the significant advance of other firewall technologies such as stateful inspection, performance or firewall configuration simulation tests [11] [7] [6].

The previously mentioned kinds of questions may also equivalently be interpreted into following kinds of questions under the graph model of the previous section such as:

1. Can a particular group of packets with some protocols and port numbers (services) reach their destined zones? Which are accepted or dropped?

2. Who can reach a particular host (zone) at certain services?

3. If we remove and add some rules, what effects will there be, or will accessibility of a particular host or zone be changed and at what services?

The above questions represent many specific questions raised in practice during security analysis since any particular questions will eventually come down to what packets from one point to another can accomplish; and what kinds of packets should not reach which zones. In the thesis, I would like to focus on providing a tool to answer these questions.

\subsection{Solutions and our contributions}

The above arguments demonstrate that answering those challenging questions is fundamentally important for firewall configuration and information security. Our goal is to provide a tool to assist with answering the suggestions.

There are many books on firewall technology and how to build firewalls. There are 
many firewall products and relevant tools on the market. However, "none seems to focus on firewall and security policy analysis tools" [11]. Under the observations of [11], most currently available tools for testing vulnerability of firewall configurations use conventional testing methods. They send and receive packets to simulate possible network traffic, observe the behaviors, and then make judgments.

These active test tools have several inherent limitations [11]:

1. They are neither complete nor efficient: when the corporate intranet is large with thousands of hosts, testing is a slow and tedious process. And certainly it is not possible to try every possible IP address and service due to computing cost. This may allow some security holes to go undetected.

2. Inconvenient and disruptive: Some authorized data being blocked wrongly may go undetected during a test. The "wait until complaints" strategy may cause inconvenience and disruption, especially for critical applications.

3. After-the-fact: testing can find new errors but cannot prove the correctness of a configuration. Detecting a problem after deployment of firewalls may be dangerous and costly since some damage may have already been done. Also errors may be hard to reproduce.

4. Some features are difficult to test: it is hard to test vulnerability to spoofing attacks because the tools may not be able to receive reply packets since the source IP addresses are faked.

Therefore [11] proposed some design goals for tools for analyzing firewall configurations that can overcome those restrictions:

1. Complete and efficient: Every possible packet is considered in the context of 
security. The time of performing this job should mainly depend on the topology and complexity of firewall rules.

2. Offline: No actual sending and receiving of packets. The analysis should accurately reflect the security policy based only on the topology and firewall rules, so the results can be obtained before the firewall actually deploys.

3. No harm: Reconfiguration of networks for active testing may make the networks vulnerable to attacks during the testing period or afterwards if loopholes opened for testing are mistakenly left open. The new tools do not need reconfiguration.

4. Easy to use: Some abstraction of results can lift the burden of checking low level firewall rules that may be different for different vendors. An interactive and graphical user interface should eliminate unnecessary difficulty related to security analysis.

So far only a few new tools have been developed. In particular, there are three major ones:

1. [11] described the design and implementation of the "FANG" (Firewall ANalysis enGine) tool which combines a graph algorithm and a rule simulator. This tool has been integrated into a firewall product successfully. After configuring multiple firewalls, a security administrator may wonder what packets can reach their destinations, and what packets should not reach certain zones. The FANG tool can make a list of what data packets reach what zones. The disadvantage of this approach is that it uses a special-purpose algorithm for creating these lists. So it is hard to add new functionality.

2. [6] Implemented an expert system or tool for analyzing firewall rules by using 
Eclipse, a kind of constraint logic programming system. The advantage of the approach is its utilization of the reasoning capability of Eclipse system. Its implementation is quite simple and fast because the major job of the implementation is just interpretation of firewall rules and topology of networks in Eclipse. So it can add functionality and capability to the tool quickly. One drawback is that the tool needs to rewrite firewall rule sets so that the rules do not overlap. The new rule sets can be much larger than the originals. This may cause difficulty for firewall administrators since query results are no longer about the actual rule sets being deployed. It is also difficult to answer questions related to firewall rule changes.

3. [7] developed a tool where a firewall rule set is represented as a Boolean function. OBDD (Ordered Binary Decision Diagram) techniques for computing with Boolean functions are used to implement the analysis tool. The examples in the paper show the usefulness of the tool to answer a broad set of questions for a firewall configuration. However, the tool is only for a single firewall rule set and cannot handle multiple firewalls.

Their tools have inherent advantages over testing tools. Details will be given in the next chapter.

In the thesis, I will present a new approach to develop a tool that overcomes all of the disadvantages or shortcomings of the above three approaches. In addition, our tool answers queries that the existing tools cannot. The tool is essentially an extension of approach 3 above to handle multiple firewalls instead of just a single firewall. The thesis is only to show the key points of our new approach and demonstrate its feasibility. It may ignore 
some implementation issues such as graphical user interface.

For example, our tool can answer queries like "what packets will be blocked by new firewall configurations and pass by the old ones", "what effects are if we remove, modify or add rules into a firewall under multiple firewalls".

Our approach is based on representing the packet data flow over networks or graph as a state transition system. Regarding a specific data packet being in a particular zone as a state, the data packet flow over networks is just like a state transition system, which evolves from one state to another as a packet travels from the source to the destination. This representation allows us to use a verification method of computer systems called model checking. A model checker takes as input a model of a system, usually as a state transition system, together with a specification of system behaviour written in some formal language, and automatically answers whether the written specification is true. In the case where the answer is false, it usually will also give a system trace exhibiting the bug. It is a proven successful tool in many system applications. However, the only use in firewall configuration analysis so far is [7]. The implementation of the tool to answer the kinds of questions given earlier for firewall configurations is quite simple and straightforward because the techniques and tools of model checking are borrowed.

We use the well-known model checking system, called SMV (Symbolic Model Verification), to implement our tool for firewall configurations. SMV is a powerful tool and has many applications in system verifications. The SMV language is very concise. Our core implementation of the tool only contains several dozen lines of codes, excluding the code directly relating to initial values like firewall rules and network topology description. SMV uses CTL (Computation Tree Logic) and LTL (Linear-time Temporal Logic) to 
specify properties which, in our case, will correspond to queries about firewall configurations. SMV will verify the properties against the state transition system and give a yes or no answer. If anything goes wrong, the counter examples given by SMV will show where the error occurs in the firewall configurations. SMV uses OBDD techniques internally. The representation will be explained later.

The purpose of the thesis is to demonstrate feasibility of this approach to firewall analysis. With this approach, the thesis makes several significant contributions:

1. Providing a new approach to develop a firewall analysis tool. The tool is very flexible in answering different kinds of questions, yet the implementation is very simple and easy to extend with new functionality (e.g. including routing information).

2. Giving a new idea to represent data packet flow over the Internet through firewalls as a state transition system that can be used for model checking.

3. Applying SMV to firewall configuration analysis at first time.

4. The ideas presented by the thesis may be applied to other problems in information security. Access control and privacy protection are just two examples of needing new techniques and tools to tackle increasingly complicated problems with confidence.

We show the tool effectiveness by testing it on a realistic, though not very large, example. In the test, the notorious "state explosion problem" is avoided, and all queries are answered quickly. 


\section{Chapter 2}

\section{Previous solutions}

Much research on firewall technology has been done [11]. Cheswick [4] claimed that most of it is not directly related to configuration problems even though that is the chief factor in the failure of firewalls.

In this chapter we describe in more detail the three main existing tools for analyzing firewall configuration that we outlined in the previous chapter. In addition, we describe a fourth related project that was done after our tool was built.

\subsection{FANG: A firewall analysis engine, a graph approach}

\subsubsection{The basic graph model}

From the perspective of a firewall administrator, networks are partitioned into zones of hosts such as DMZ, corporate intranet, human resources, and the external Internet, all with disjoint IP address sets. The zones are connected through gateways, which have an interface where firewalls sit in between each adjacent zone. We can have a gateway-zone graph, where vertices consist of gateways and zones, and where the interfaces, with 
firewall rule sets attached, form edges.

The goal of FANG is to provide a tool to answer a certain class of questions about firewall configuration. It has a query/answer scheme that lists what kinds of packets can reach particular zones of concern to the firewall administrator.

\subsubsection{Query/answer scheme}

A query in Fang consists of three elements:

- Source group hosts: represented by their IP addresses

- Destination group hosts: represented by their IP addresses

- Service group: represented by a combination of protocol identities such as TCP/UDP, and source and destination port numbers.

When a specific query is made, the tool can give a list of what kinds of packets in the subset of the query are permitted with the given firewall configurations. We give an example to demonstrate the idea [11].

The following Figure 2.1 shows a small corporate network with firewalls [11]. The external firewall, which has two interfaces, protects the corporate network from the public Internet. The servers in the DMZ zone provide the corporation's services needed by the public via the Internet. The internal firewall, which has three interfaces, protects the corporation's internal networks from public access. Any compromise in the DMZ zone would not spread over the internal networks. The "control" machine in the corporate zone 
provides administration service, which may have some special privilege to administrate the whole corporation networks. This zone should have minimal access for security reasons.

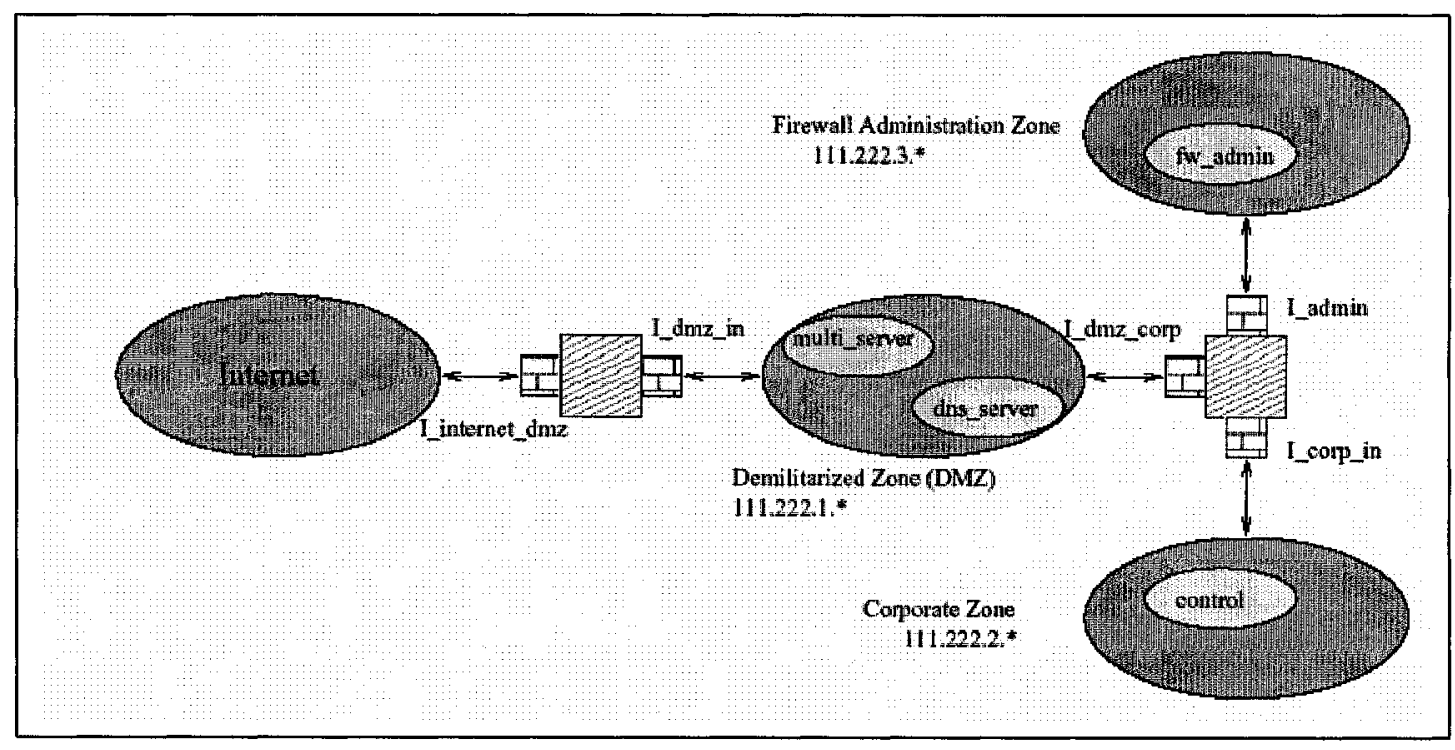

Figure 2.1 A corporation networks with multi firewalls

Let us omit the formal specification of security policy, network topology and interface addresses presented in [11] for simplicity. The firewall rule sets are omitted in the paper [11]. These omissions do not hinder understanding of what the FANG tool can do and how.

Query 1: What services are allowed between the corporate zone and DMZ.

Figure 2.2 [11] shows FANG's answer to the query of firewall configurations. The services of servers in the DMZ are available to all the hosts in the corporation zone, but TCP connections to the servers can only be opened by the "control" computer. Note that all the concerned objects have distinct names that are more meaningful than raw IP addresses. The naming is only a new layer for the benefit of the user. The raw numbers are accessible just by clicking a mouse. 


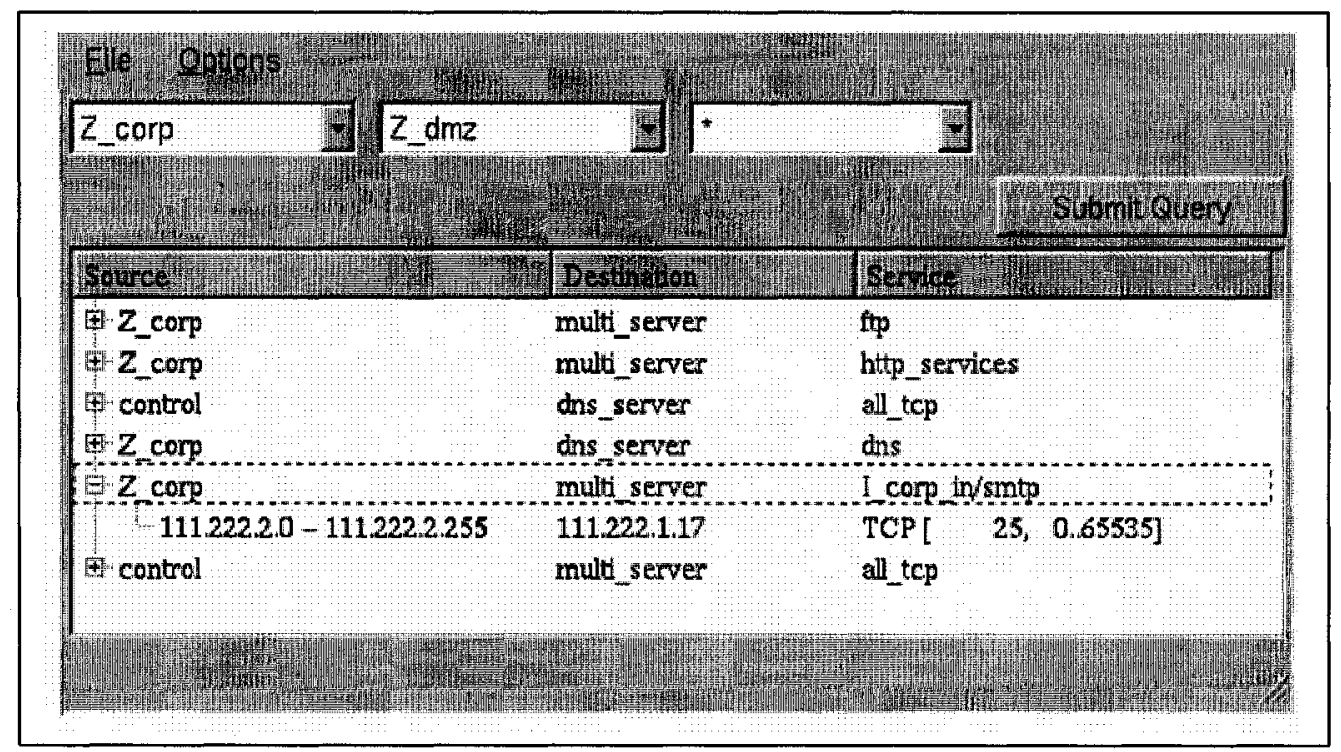

Figure 2.2, A simple query example of FANG.

Query 2: How much access does the Internet have to the internal network?

Figure 2.3 shows some restriction on packets from the Internet. Note the FANG assumes that any host in a zone can speak freely with any host in the same zone. The last line in Figure 2.3 demonstrates a weakness of the firewall configuration. The line says that any host from the public Internet can talk with the inner interface of the external firewall, I_DMZ_In, for any service. This happens because the outer interface, I_Internet_DMZ, allows any packets into the gateway. If the rule sets attached to $I_{-} D M Z_{-}$in are switched with I_Internet_DMZ, there will be a better configuration in this case. 


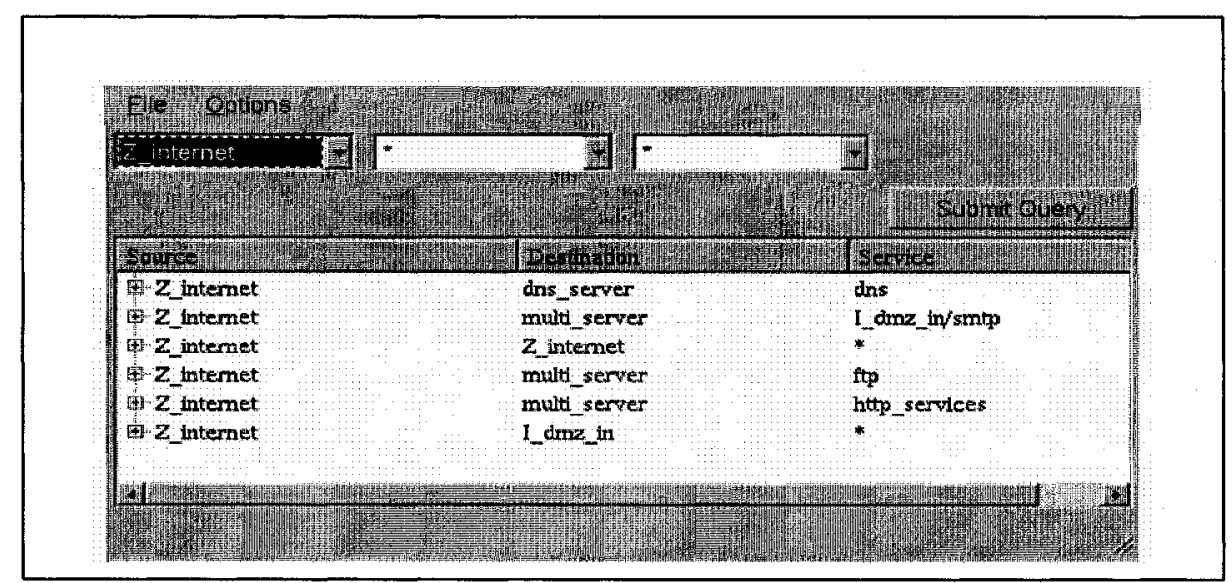

Figure 2.3 Another query example of FANG

Query 3: No host from public Internet can reach the firewall administrator zone, even with spoofing attacks.

The results are shown in Figure 2.4. Spoofing means that the source IP address in a packet is faked. The tool uses an extra field to show the real IP address, namely, where the packet comes from. The list in Figure 2.4 shows that packets from the Internet with faked source address 111.222.1.1 can reach the firewall administrator zone. Once the vulnerability revealed, it is easy to fix it.

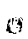

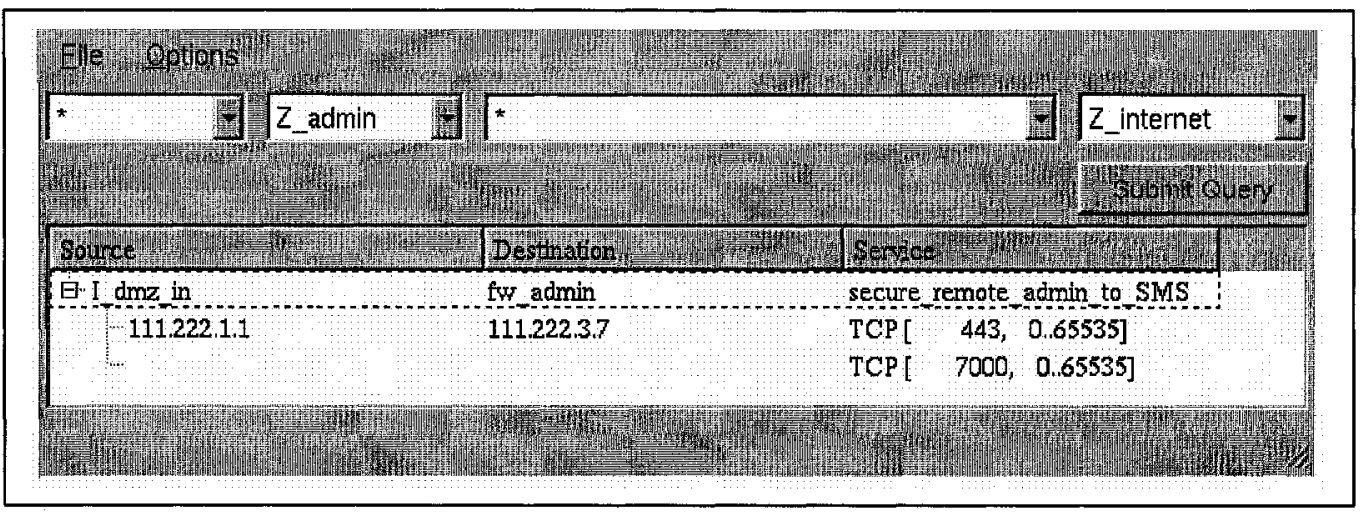

Figure 2.4 A spoofing example of FANG 


\subsubsection{Outline of FANG implementation}

The first step a FANG tool user needs to do is to specify the tool network's topology and associated firewall rules. The topology description file can be written in a subset of Firmato's MDL language [2]. The topology only reflects the structure of zones and firewalls. It does not reflect the actual topology of routers and switches. This model is represented as a graph. As part of the topology file, names of the multi-firewall configuration files that contain rules are specified. So the tool can understand which firewall rule set attaches to which interface, or edge in the graph model. Once the topology file is ready, the tool will read and parse it, and construct a graph. Then it will parse these firewall configuration files which are highly vender-specific and forms its internal firewall rule set structure with fields ( sourceaddress, destinationaddress, servicegroup, direction, action )

Please refer Figure 2.5 for the process.

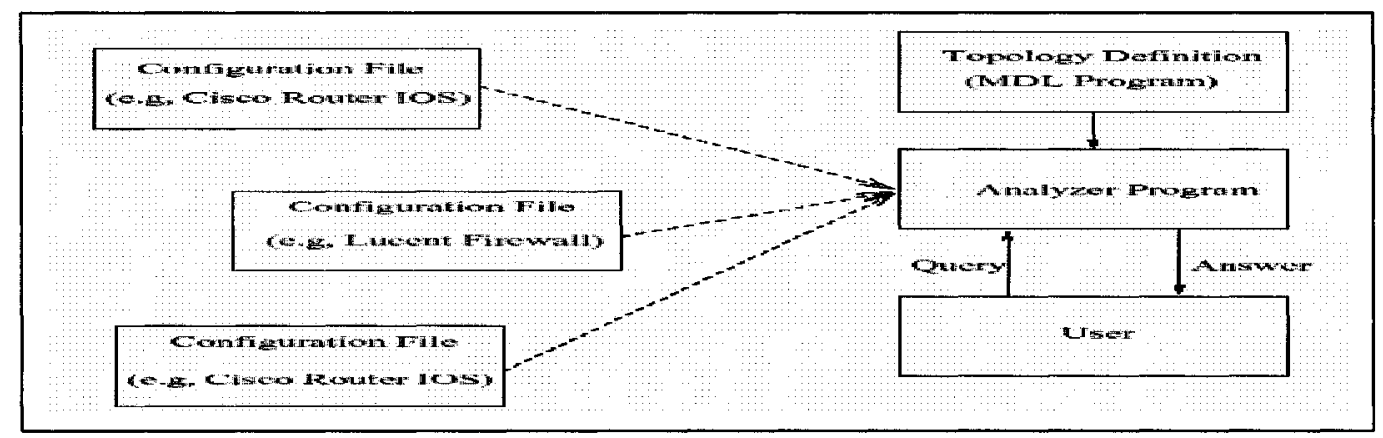

Figure 2.5 FANG architecture and data flow

Now the FANG GUI is launched and ready to accept user queries. After a user query is submitted, a graph algorithm and a rule-base simulator are used to obtain a list of accepted packets permitted in the set of the query. 
The user's query typically consists of a group of source and destination IP addresses and a group of services. The graph algorithm initially starts at the zone containing the query's source host group. If the source host group, namely their IP addresses, span over several zones, it is broken into disjoint host groups, each of which is contained in a single zone, and the query is broken into several subqueries. The same algorithm applies to each of them. Each query is propagated into the neighbor zones of the current zone across edges. During the propagation, the query is usually broken into sub queries because packets represented by some portion of the query are blocked by the firewall rules associated with the edge. The rule-base simulator does this for each edge. For instance, the query may be (corporate_net, Internet, ${ }^{*}$ ) and the firewall says that only http and smtp are allowed. After crossing the edge, the query is broken into two subqueries, respectively (corporate_net, Internet, http) and (corporate_net, Internet, smtp). The algorithm continues in the same manner for each of the queries until the full graph is explored, i.e. all paths have been tried from source addresses to destination addresses of the queries. The algorithm also has some tactics to reduce computing cost. The final step is to collect the results by looking at the queries in all zones that have reached their destination hosts and presenting them in a user-friendly tabular form.

A minor modification of the above algorithm can deal with spoofing attacks. Spoofing means that the source IP address of a packet is faked. So a new parameter is added into the query structure to indicate the true IP address of a packet. In spoofing attack situation, the algorithm may start from the zone that contains the real IP addresses. The rest of process stays almost identical. 


\subsection{Expert system approach}

\subsubsection{Introduction}

Expert systems are computer applications typically focusing on problems that usually require professional expertise or human reasoning capability. Expert systems have become powerful tools to assist solving problems in a wide range of areas including planning, scheduling, decision support, and process control, etc. A good example of expert system applications in information security is in the field of intrusion detection [1]. Expert systems can also help firewall administrators answer the challenging questions raised before: what kinds of packets can travel to their destinations under the restriction of firewalls.

\subsubsection{Implementation of an expert system for firewall configuration}

Expert systems are typically constructed with three major components: a knowledge base, an inference engine and a user interface. The knowledge base consists of facts and logical rules about a problem domain. The inference engine solves problems and deduces knowledge by making logical inferences based on facts and rules stored in the knowledge base. The user interface handles the system input and output.

The expert system in [6] for analyzing firewall rules is implemented in Eclipse, a constraint logic programming language and system based on Prolog. Logic programming 
solves problems by using logic inference based on facts and rules that describe a solution instead of requiring computational details or steps. Constraint logic programming is logic programming extended with constraint satisfaction.

The knowledge base should contain the following major facts and rules:

- Network topology and interface associated with firewall rules.

- Firewall rule set or access list: the restriction of packet flow.

- Packet space: the domain knowledge.

The basic concept is the packet space. It is represented by 6-tuples ( protocol, source IP address, destination IP address, source port, destination port, flags). The following predicate describes the 6-dimensional finite, discrete packet space:

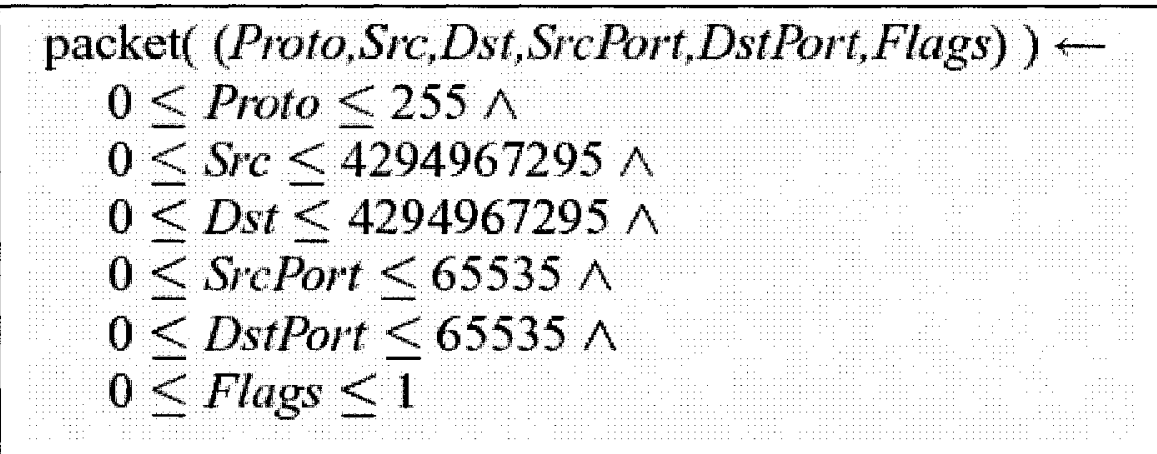

Figure 2.6 Packet representation in the system using Eclipse

More constraints are used to express parts of packet space. The following predicate declares the set of TCP packets (protocol=6) whose destination is the HTTP port (80) of host 10.0.0.1 (167772161 as a single integer):

Packet(Proto, Src, Dst, SrcPort, DstPort, Flags)^

$$
\text { Proto }=6^{\wedge} \text { Dst }=167772161^{\wedge} \text { DstPort }=80
$$


The direct implementation of order-sensitive firewall rules of packet filtering is counterintuitive and inefficient in Eclipse, so the implementation firstly "de-correlates", or splits, the rules into non-overlapping rules. In another words, any particular packet may match no more than one rule after the firewall rule set is split, and the new rule set will no longer be order sensitive. This may present a problem because firewall administrators have to adjust the previous, order sensitive rule set rather than the new split rule set when they reconfigure firewalls.

The rules added into the knowledge base are like the following example:

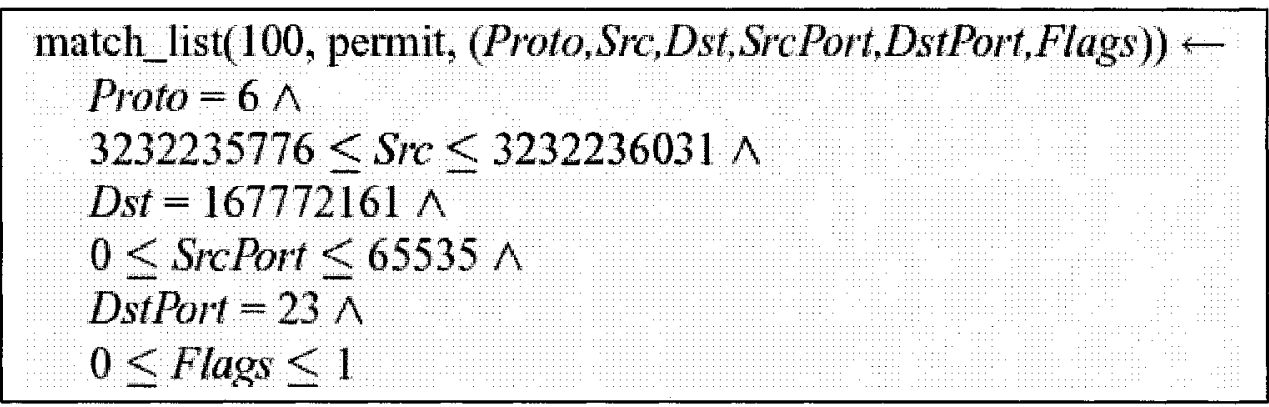

Figure 2.7 Match list predicate in the expert system

The predicate is for the rule:

Accesslist 100 permit tcp 192.168.1.0 255.255.255.0 host 10.0.0.1 eq 23

The rule says the packets from hosts $192.168 .1 .0 / 24$ to the host 10.0.0.1 with TCP connection at port 23 are permitted.

The network topology and interface information are represented as predicates:

1) Network (interface, network). This structure denotes which interface is connected to which network, or its attached IP addresses.

2) Network-Internet (interface). This indicates which interface is connected to the Internet. It tells the inference engine which firewall rule sets control packet flow to 
and from the Internet. This is a special case of the above since the Internet is not needed to allocate IP addresses.

The knowledge base of the system can be extended easily and flexibly.

\subsubsection{User interface and example}

The user interface currently implemented is a simple command-type one. The following example of the paper demonstrates its main flavor. The following Figure 2.8 is a simple example of a network.

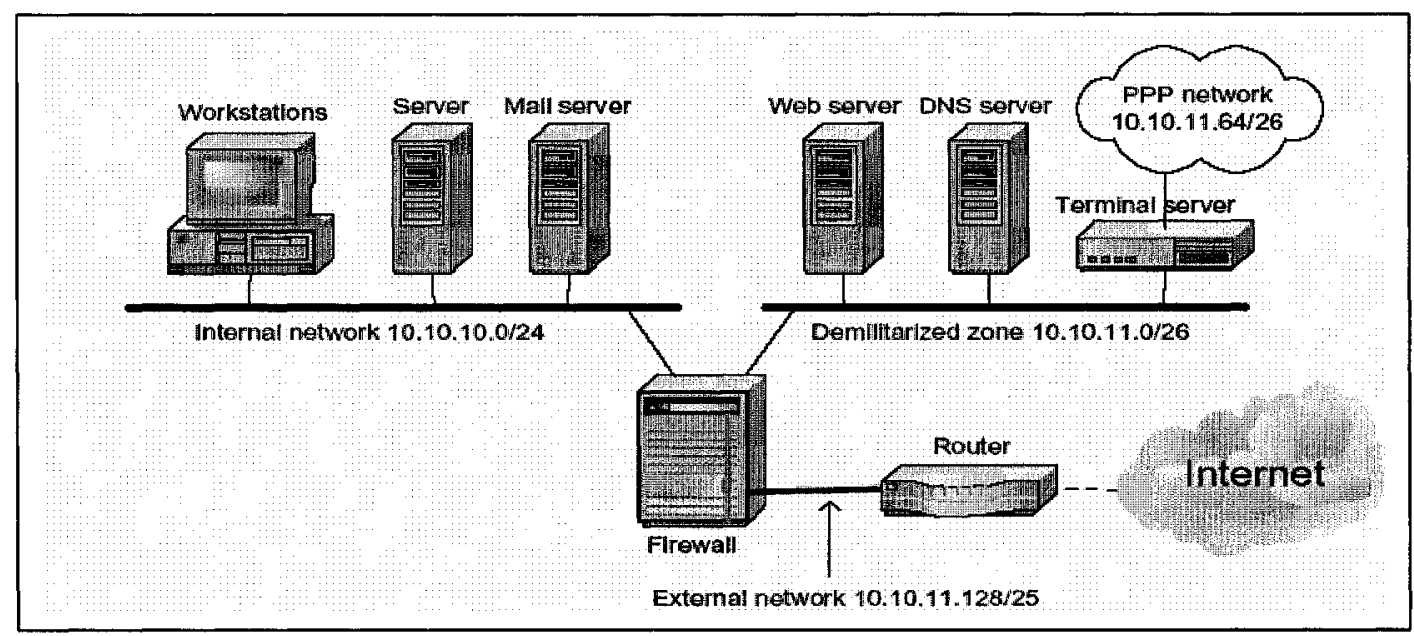

Figure 2.8 The network of a small company

The network has three zones: internal network, $\mathrm{DMZ}$ and external networks. When the expert system is used, network topology, interface and firewall rule files are opened and parsed. The information is contained in a file for reuse. A scenario looks like Figure 2.9: 


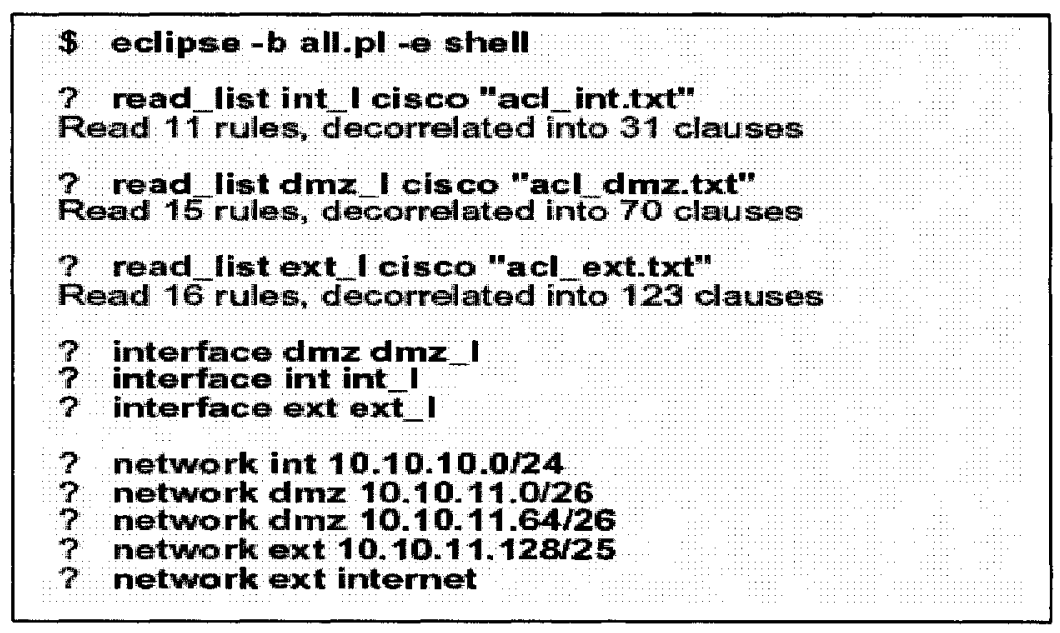

Figure 2.9 An example of command list in the expert system using Eclipse

In the above example, 16 overlapped rules are split into 123 non-overlapped rules. The significant increase of rule number may not be tolerated in actual firewalls for performance concerns but is fine for security analysis.

Once the knowledge base is established, the tool is ready to answer questions. The following Figure 2.10 demonstrates what kinds of packets can reach the services on the web server in DMZ, 10.10.11.8.

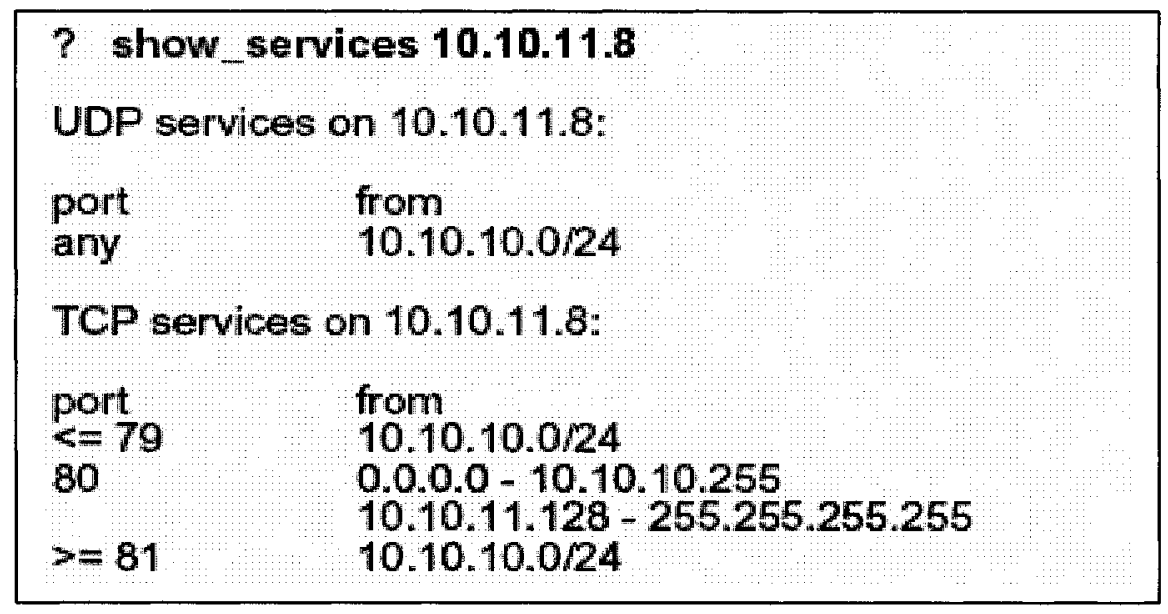

Figure 2.10 A query example using the expert system 
This kind of information can help a security analyst identify the potential weakness. Without the proper tool, it is hard to get this kind of information under sophisticated circumstances. 


\subsection{Boolean expression approach}

\subsubsection{Introduction}

This section will present Hazelhursts' work of using OBDDs to assist in analyzing firewall rule sets [7]. The paper did not deal with multi firewall situation, so it has no description of network topology. It is targeted at a single firewall rule set and is concerned with what kinds of packets can be dropped or passed by the rule set. The basic strategy of this approach is to use Boolean formulae to represent packets and rules. Then the OBDD (Ordered Binary Decision Diagram) technique for Boolean formulae, widely used in computer aided verification, is used in the analysis of firewall security, specifically in answering those questions relating what kinds of packets will drop or pass. We will illustrate the main idea of this approach and its benefits by using examples given by the paper [7].

\subsubsection{Ordered Binary Decision Diagrams (OBDD)}

Before we discuss how to convert rules into a Boolean function, we will briefly explain about the OBDDs (Ordered Binary Decision Diagrams). OBDDs are a technique of representing Boolean functions in a compact way and supporting efficient Boolean operations in many cases. It has made model checking workable for verifying a large scale of practical systems. For details on OBDDs, refer to the book of Huth and Ryan [10]. 
A Boolean formula over a set of Boolean variables can be viewed as a Boolean valued function with Boolean inputs. Boolean functions and their operations play important roles in modeling and reasoning for many systems. A common way to represent a Boolean function is to show its truth table. However this is not a good representation for solving practical problems due to its exponential complexity. Another representation of a Boolean function is a binary decision tree. Let us look at an example of these representations. Suppose we have a Boolean function $f(x, y)$, where $x$ and $y$ are Boolean variables. Its truth table is showed in following Figure 2.11:

\begin{tabular}{|c|c|c|}
\hline$x$ & $y$ & $f(x, y)$ \\
\hline 0 & 0 & 1 \\
\hline 0 & 1 & 0 \\
\hline 1 & 0 & 0 \\
\hline 1 & 1 & 0 \\
\hline
\end{tabular}

Figure $2.11 \mathrm{~A}$ truth table of boolean function

To find $f(1,0)$, locate the row $x=1$ and $y=0$, get the value 0 or false. Its equivalent binary decision tree is the following figure.

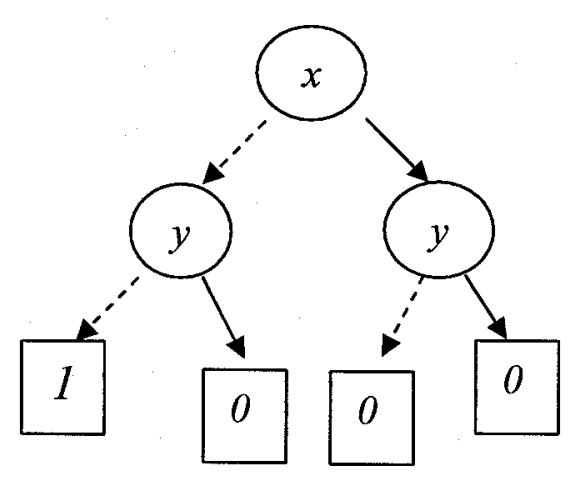

Figure 2.12 A binary decision tree of Boolean function 
In the decision tree, the dashed arrow out of a node represents the case where its labeled Boolean variable is 0 , or false, and the solid arrow the case where it is 1 or true. To find $f(1,0)$, start at the root node $x$, follow the solid arrow and reach the right node $y$, then follow the dashed arrow to the third leaf node from the left, read the labeled value and find that the value of $f(1,0)$ is 0 or false.

Logical operations on Boolean formulae, such as conjunction and disjunction, can be implemented for the OBDD representation. The key idea for making these operations efficient is removal of redundancy. For instance, the right node labeled $y$ has no new contribution to the Boolean function $f(x, y)$. In other words, once the variable $x$ is $I, f(x, y)$ equal 0 no matter what $y$ is. So the subtree can be replaced by a single node labeled 0 .

There are three simple techniques to reduce redundancy in decision trees. With reduction the binary decision tree is no longer a tree and becomes a $\mathrm{BDD}$, i.e. a binary decision diagram [10].

Firstly, it is easy to see that the leaf nodes are usually redundant. Only one copy of a 1-node and a 0-node are needed. All other leaf nodes can be removed and all the lines directed to these nodes can be redirected.

Secondly, if both outgoing edges of a node $n$ point to the same node $m$, the node $n$ is useless: eliminate it and direct all incoming edge of node $n$ to the node $m$.

Thirdly, if two distinct nodes $n$ and $m$ are parents of identically structural sub BDDs, remove one node; direct all incoming edges of the node to the other node.

The above three simple processes can reduce the redundancy of a BDD. Repeat the processes until no more reduction is possible. Then the BDD is called a reduced BDD. For instance, the above binary tree example can be reduced to the BDD in Figure 2.13. 


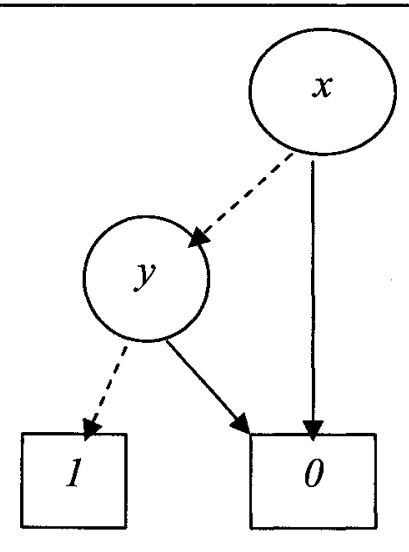

Figure 2.13 A Binary Decision Diagram (BDD) of Boolean function

A reduced BDD is a compact way to represent a Boolean function. However, in order to make Boolean operations over BDDs practical the BDD should also be ordered:

- For any two Boolean variables $x$ and $y$ in a BDD, if $x$ is before $y$ in one path from the root to a leaf, $x$ is always before $y$ in any other path from the root to a leaf.

Reduced OBDDs have some very useful properties. For example, two Boolean formulae are equivalent if and only if their respective reduced OBDDs are identical when their variables are in same order. Operations on Boolean functions can be transformed efficiently into corresponding operations performed on OBDDs. The OBDD technique represented a major break-through in practice due to its compact structure and efficient operations [10].

The ordering of Boolean variables in an OBDD may have a big impact on the size, or complexity of the OBDD. For instance, there is a Boolean function where in one order of Boolean variables the size of OBDD is linear in the number of Boolean variables while in another order it is exponential [10]. There are some good heuristics to find a good order of 
Boolean variables for OBDD. However, it is a NP-Complete problem to find an optimal order to make the size of its OBDD minimal. Besides, you can not have polynomial size of OBDD for some problems. As an example, R.E.Bryant proved that no matter what variable order is chosen, integer multiplication over OBDDs is exponential [P349, 10].

In summary, the representation of an OBDD and performance of its operations are not guaranteed good in theory. However, in practice, OBDDs have demonstrated excellent performance for many large systems. It has proven to be a powerful tool, although it is not always efficient and may fail in some situations. Hazelhurst [7] applied the technique to analyzing a firewall rule set for the first time. Some experiments demonstrated that it is a promising approach.

\subsubsection{Algorithms for analyzing a rule set}

This section describes how a rule set can be converted into a Boolean formula and hence an OBDD.

The first important idea is to represent an integer as a Boolean formula. An integer is equivalent as a Boolean formula or a specific value of Boolean variables. It is well known that we use binary bits to represent integers. For instance, we can use 3 binary bits to represent 8 integers from 0 to 7 . They are also can be represented by 3 Boolean variables $x$, $y, z$. For example the binary form of number 3 is 011 , so it can be represented by a Boolean vector $(x, y, z)=(0,1,1)$, (false. true, true $))$. This number can also be represented as a Boolean function $f(x, y, z)=x^{\prime} y z,((\operatorname{not}(x))$ and $(y)$ and $(z))$, where symbol " ' " represents 
negation of Boolean variable $x$ since $(x, y, z)=(0,1,1)$ is the only value to make the Boolean function true.

Now how can we represent a set of integers in Boolean formula? Suppose $j$ as an integer variable. The condition $j=2$ or $j=3$ can be represented by the Boolean formula, $f(x, y, z)=x^{\prime} y z^{\prime}+x^{\prime} y z$, which may be reduced into a simpler form of the Boolean formula, $f(x, y, z)=,x^{\prime} y$. To make formula $x^{\prime} y$ true, $x$ must be 0 and $y$ must be 1 ; the Boolean variable $z$ is free. This means this formula can represent two integers. When $z$ is 0 , the represented integer is 2; and when $z$ is 1 , the represented integer is 3 . For a range of integers, a Boolean formula can be formed by the disjunction operation of Boolean formulae for individuals in the range. Of course, there are some transformations that can be applied to avoid the naïve and inefficient disjunction for a group of integers.

Let us use a CISCO access rule as an example to show what is a rule and how to convert a rule into a Boolean formula. The following is a typical rule [7]:

Access-list 101 permit tcp

20.9.17.0 255.255.255.255

$121.11 .127 .20 \quad 255.255 .255 .0$

range 2350

The key elements of the rule are:

- Permit or reject: when packets match the rule the action is taken.

- The protocol of the packet: in the example, it is TCP, an integer.

- Source address: IP address, four segments, each is an integer between 0 and 255 .

- The mask of the source address: four segments. 
- Destination address: IP address.

- The mask of the source address: four segments.

- The range of port addresses. In IP packets, there have source port and destination port numbers; but in most time they are same.

A 32 bit IP address can be viewed as an integer. How to interpret integers into values of Boolean variables or Boolean formulae is already described. So we need to understand how to treat the source address and destination part with the masks. The masked IP addresses represent a group or a range of integers. Both an IP address and its mask are 32 bits long. In the rule the mask bits decide which bit of a packet should match the respective bit of the rule and which should be ignored. Suppose $s$ is a bit of the IP address in the rule and $m$ is the bit in the same position in the mask. The bit $p$ is the corresponding bit in a specific packet traveling through a certain firewall or zone. The packet matches the rule in the position for the bit if the following Boolean formula is true:

$$
(s \text { and } m)=(p \text { and } m)
$$

If the mask bit $m$ is true, the bit of the packet must be same as the bit of the rule; otherwise the bit of the packet can be free or any value, namely, either 1 or 0 .

A packet matches the rule for the source address part if all the 32 bits of the packet's source address match the rule in their respective positions. The same principle applies to the destination address part.

To the example rule, the source address is 20.9 .17 .8 and the mask is 255.255 .255 .255 . This means a packet must come exactly from a host with IP address 20.9.17.8 when the packet matches the rule. Similarly, the destination address is 121.11 .127 .20 and the mask is 
255.255.255.0. This means a packet must go to a host with first 24 bits of IP address being 121.11.127. With the method of converting integers into Boolean formulae described before and conjunction of these Boolean formulae we can easily convert a rule into a Boolean formula. A specific IP address can be regarded as an integer. The IP address representation along with its mask simply stands for a set of 32-bit IP addresses or 32-bit integers.

The following pseudo code description of an algorithm to convert an ordered rule set into a Boolean formula is due to Hazelhurst [7].

\{

cvtrule is a function of converting a rule into a Boolean formula;

ctvruleset is a function of converting a rule set into a Boolean formula;

currrule is a variable for a rule; and

ruleset and restset are container variables for a set of rules which are first order sensitive.

Function ctvruleset(ruleset)

\{

if ruleset is empty return false or $0 / *$ default action reject if no rule matched*/

else

let currule $:=$ first rule of ruleset

let restset $:=$ rest part of the ruleset

if the action of currrule is a permit

return (ctvrule(currrule) or ctvruleset(restset))

endif 


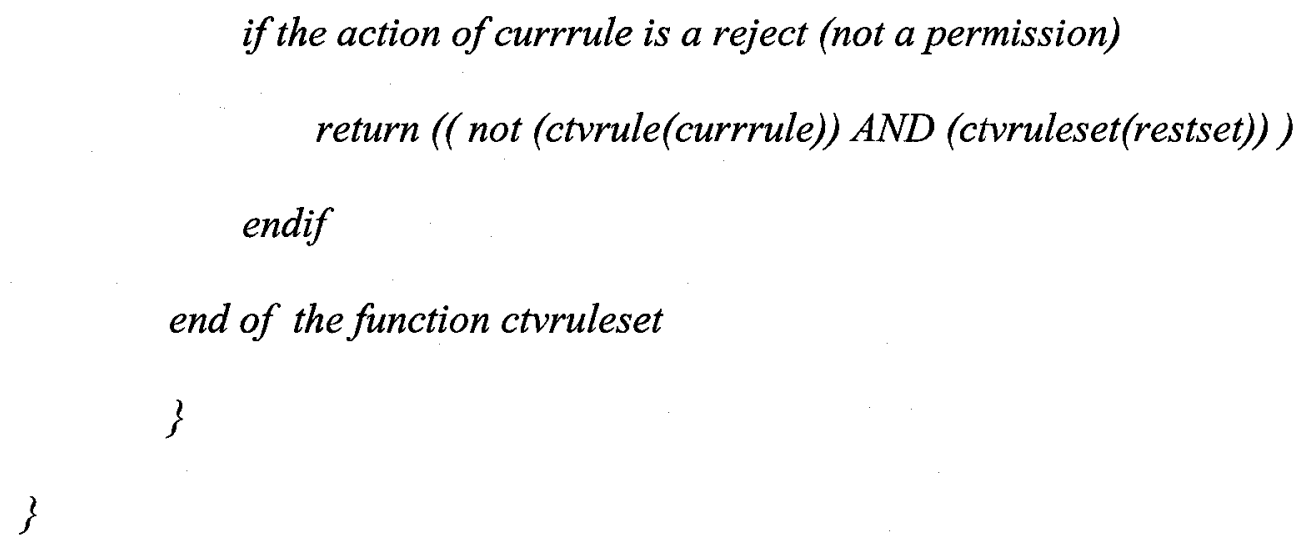

if the action of currule is a reject (not a permission) return (( not (ctvrule(currrule)) AND (ctvruleset(restset))) endif end of the function ctvruleset \}

The actual implementation used the Voss system, which has heuristics for finding good variable orderings for its internal OBDD representation. Experiments have showed the approach also has high performance although much more tests in practical environments needed to confirm that.

\subsubsection{Examples}

This section uses examples to show the usefulness of the tool. After the conversion of the firewall rule set, the next step is to answer questions raised in the analysis of the rule set, such as the following:

- Do we accept packets on port 25? If so what kinds of packets?

- What packets can reach our web servers? How about the SMTP server?

These queries can be transformed into a Boolean formula by using previous described techniques. These queries add some conditions or restrictions to the rule set. In other words, users focus on examining a particular set of packets permitted by the rule set using the queries. Hence we can get the resulting Boolean formula by conjoining the Boolean 
formulae converted from a query with the rule set. Operations such as conjunction and disjunction are generally efficient by using OBBDs although there is no guarantee of performance in theory.

One more key step needed to make the tool useful is to present results in a user friendly way. You can not let users look directly at an OBDD which is machine friendly but unintelligible to users. The tool presents the OBDD in a tabular form. The paper did not show how to do that, but the interpretation can be quite easy. The basic idea is to take a path from root to a leaf and write the corresponding Boolean variable values in a bit string representing an integer. If Boolean variables are missing in the path, it means they are free variables and they can be either 1 or 0 . So we get the integer in binary form with a range or several ranges. Then it is transformed into a decimal form that is human friendly in number. The numbers are presented in ranges generally; please refer to the following example.

The tool provides command type user interface to assist user to analyze firewall rules. The basic input structure is:

: sc [Proto, Port] cond;

This first part is a command and second part is for the display order of columns in a tabular form. The third part is a Boolean condition converted from a user query. A few examples copied from the paper can show what the tool can do, See Figure 2.14. 
- What type of udp packets do we accept?

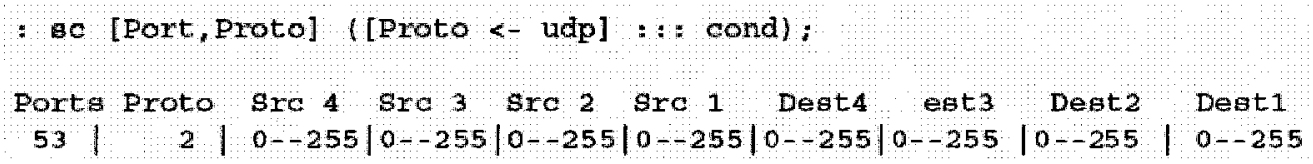

- What packets do we accept which have the first segment of the destination address of 121 and which are not icmp packets?

\& sc [Port, Proto] ([Dest1<-120, Nor (Proto<-1cmp)] ; : , cond);

Porta Proto SrC 4 Sro 3 Src 2 Sre 1 Dest 4 Dest 3 Dest 2 Dest 1

$0--19|\quad 1| 0--255|0-255| 0-255|0-255| 0--255|0--255| 0-255 \mid 120$

$20--21|\quad 1| 0-255|0--255| 0--255|0--255| 0--255|0--255| 0--255 \mid 120$

$3|0--255| 0--255|0--255| 0--255|\quad 3| \quad 112|\quad 17| 120$

$22|\quad 1| 0-255|0-255| 0-255|0--255| 0-255|0-255| 0-255 \mid 120$

$3|\quad 9| \quad 0|\quad 20| \quad 120|0--255| 0-255|0--255| 120$

$23--24|\quad 1| 0--255|0--255| 0--255|0--255| 0-255|0--255| 0-255 \mid 120$

-...*.

Figure 2.14 Tabular form of results of a query of a firewall rule set

In Figure 2.14, the third part ([ Proto <- $u d p]::$ cond) of the first query says that the packets are $u d p$ packets. The Src denotes source addresses of packets, where the number shows which part of the 4-byte IP address. The Dest denotes destination addresses.

With the approach of using Boolean formulae and OBDDs, it is easy and efficient to implement methods for analyzing changes to the firewall rule set. For example, what will happen if a rule is changed, removed, added or moved? The answer to the change will present a view of the impact on security. For example, the following example shows the packets blocked by a new rule set but permitted by an old rule set. See Figure 2.15: 


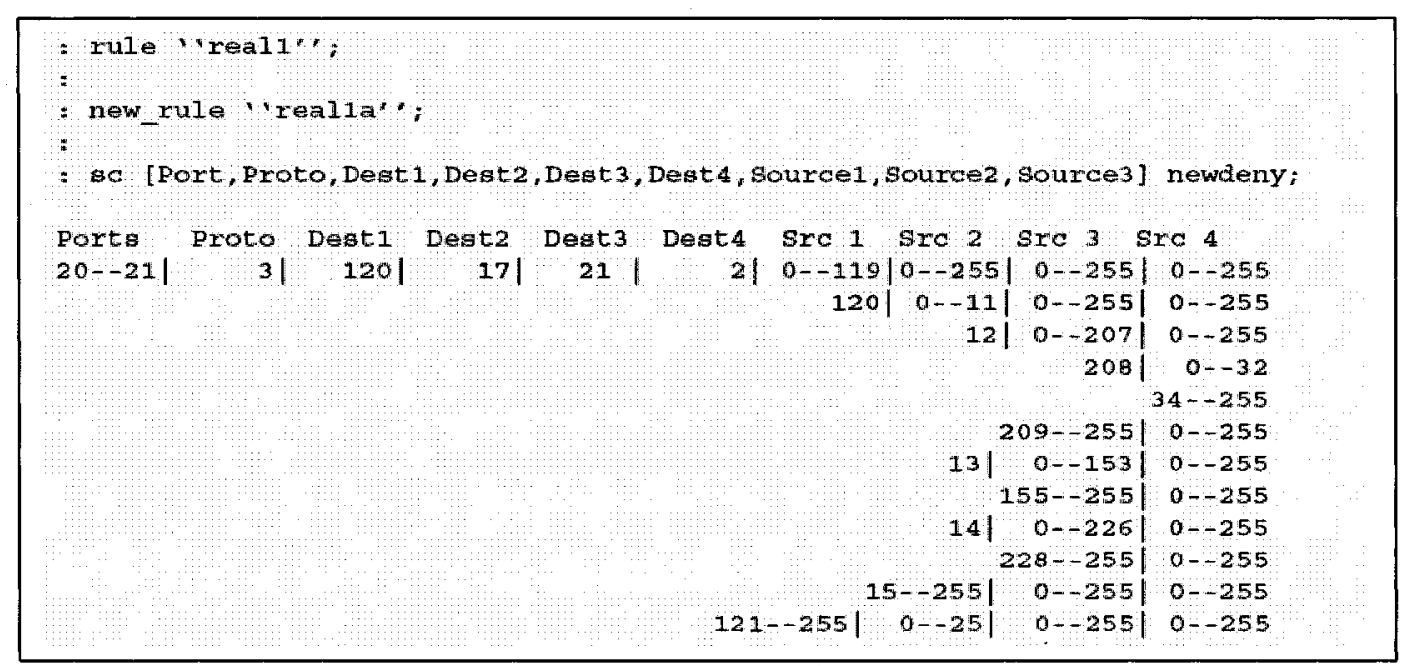

Figure 2.15 query of consequences with firewall rule change

The second part, $[\ldots]$, is for the display order of output. The third part newdeny is the query condition.

Automatic detection of redundant rules and an improved graphical user interface also has been implemented.

With this approach, a key point is that much can be accomplished with little effort because techniques developed in computer-aided formal verification can be applied. The implementation is much more flexible than other two approaches. However, the tool only deals with one rule set. It cannot be directly applied to multi firewall configuration situation, which dominates in reality. The more complicated firewall configurations are in practice, the more security responsibility firewall administrators have. And the harder it is to analyze firewall configurations, the easier it is to make a mistake. The thesis provides a solution to deal with the situation. 


\subsection{FIREMAN}

\subsubsection{Introduction}

FIREMAN [15] is a toolkit for firewall modelling and analysis. Our tool was developed before FIREMAN, but for completeness we will compare our work with it.

\subsubsection{Misconfigurations}

The paper discussed some misconfigurations of firewalls FIREMAN can fix and classified them as follows:

1. Policy violations: What packets should be passed (a white list) and blocked ( a black list) are often security policy to be enforced by firewall configurations. If firewalls are configured incorrectly, the tool can detect errors based on the lists.

2. Inconsistencies: A firewall administrator's intention should be consistent. Inconsistencies in configurations often indicate something is probably wrong.

- Intra-firewall inconsistencies: These happen within a firewall. There are three types of inconsistencies.

1. Shadowing: The set of packets matched by a rule is a subset of packets matched by preceding rules. Their actions of rules are often different. The inconsistencies often mean that some mistakes are made. 
2. Generalization: This is opposite to shadowing. The set of packets matched by a rule is a superset of packets matched by preceding rules.

3. Correlation: The set of packets matched by a rule intersects the set of packets matched by preceding rules and the action of the current rule is different.

- Inter-firewall inconsistencies: A packet may survive several firewalls to reach its destination. If a packet is blocked by an upstream firewall but passed by a downstream firewall, errors may take place.

- Cross-path inconsistencies: A packet may have several paths from its source to its destination. If the packet passes one path and is blocked by another path, there may be an error.

The paper also discussed inefficiency of firewall configurations that may give a hint to rewrite a large amount of firewall rules into compact and organized rules.

FIREMAN used BuDDY, an OBDD library, to implement rule lists. The following figure shows some problems discovered in a production firewall configuration.

\begin{tabular}{|c|c|c|c|c|c|c|}
\hline Firewall & Product & \#ACLs & \#rules & $P$ & $C$ & $E$ \\
\hline PIX1 & PLX 6.03 & 7 & 249 & 3 & 16 & 2 \\
\hline BSD1 & BSD PF & 2 & 94 & 3 & 0 & 0 \\
\hline PIX2 & PLX 6.03 & 3 & 36 & 2 & 0 & 5 \\
\hline
\end{tabular}

Figure 2.16 Firewall files and misconfigurations

In Figure 2.16, the number of firewall rule lists is in the \#ACL column. The columns $\mathrm{P}$, $\mathrm{C}$, and $\mathrm{E}$ represent the number of policy violations, inconsistencies, and inefficiencies respectively. 


\subsubsection{Comparison with our approach}

Most of FIREMAN's functionality can be achieved in our tool. For policy violation detection, the white list and black list are just normal queries which can be verified by our tool. In case of errors in firewall configurations, our tool would give counter examples to show the location of the error. For intra-firewall inconsistency detection, the work of FIREMAN can be achieved by the approach of [7], although this is not discussed explicitly in that paper. For example, shadowing inconsistencies are similar to the kind of redundancies dealt with in [7]. The other inconsistencies could be easily implemented based on the ideas of [7]. Our tool does not deal with inter-firewall inconsistencies, however we can extend our tool easily to deal with the problem. Cross-path inconsistencies are just some properties claiming that Boolean formulae (specified by CTL) are true in some path and false in another.

In summary, FIREMAN is mostly a special case of our tool. With a fairly small amount of work, our tool could be extending to include all the features in [15]. 


\section{Chapter 3}

\section{Philosophy of verification approach for multi firewall configurations}

In this thesis we describe a new tool that meets the requirements proposed by [11], improves on existing tools, and can be developed quickly. This section will discuss the basic theory and background of our approach. Next section will give the implementation framework.

The key idea of the approach is inspired by the analogy of data packet flow and state transition systems. In a dynamic view, packets flow over networks from their starting zone to their destination while some are blocked or dropped by appropriate firewall rules in intermediate zones. We can regard the packet flow over the graph as a state transition system, where a particular packet goes between zones during its journey from its starting zone to its ending zone. We view the particular packet being in a particular zone as a state. This transformation gives us a huge space of states but it enables us to utilize the state-of-art techniques proven in model checking. The key questions in firewall configurations concern what packets should or should not reach certain zones. These can be expressed as logical properties of states and transitions that can be verified by using model checking methods. The verification process will verify correctness or reveal weaknesses of firewall configurations. 
Before we proceed with our approach, a brief introduction to model checking is necessary. It is greatly desirable to be able to formally verify the correctness of a computer system because testing has proven to be insufficient for debugging certain kinds of improtant systems and applications [10]. One important verification method is called model checking. A computer can be regarded as a state transition system where a computer system evolves from state to state. A model checker takes two inputs:

1. A model: a state transition system or its subsystem from some problem domain. This is a model of a practical system people want to build.

2. Properties: What should or should not happen? These are often specified as temporal logic formulae.

The model checking system takes the two inputs and tells users whether the properties are true or false. The model checking system can also give counter examples or values to demonstrate how the system goes wrong, when one of the desired properties is false. Two common languages to specify the properties are CTL (Computation Tree Logic) and LTL (Linear-time Temporal Logic), both of which have proven to be very useful in practice [10]. It is obvious that the size of the state space is a paramount challenge since it is usually huge in practical systems. Luckily OBDDs are a powerful tool available to meet the challenge and have proven very effective in many systems, although they are not always guaranteed to be effective [10].

In order to utilize OBDDs, it is necessary to view a state transition system as a Boolean function. Most model checkers, including the one we used (SMV), automatically generate the Boolean function representation from a high level description of the system in a conventional programming language-like syntax. For details on this, see the book [10]. 
We view the data packet flow over networks as a state transition system where a state corresponds to a packet's position, or location in the network. In general, the initial state is where the packet starts from the source zone specified by the source address in the packet. The next state is either the position (or zone) the packet can go to from the current position, or the destination position of the packet once it has arrived there. As for queries, they can be easily interpreted as Boolean formulas. The model checking system can verify these formulas.

This thesis will use a well-known model checker, SMV. SMV stands for Symbolic Model Verifier. We use a free research version of SMV from Cadence Berkeley Labs.

We give a brief introduction to SMV. For more on SMV, see [10]. The subsequent discussion does not need much SMV background but having some understanding of a programming language is useful. Some relevant details will be explained in the context of the implementation.

The SMV system is a tool for model checking. It has a language to describe a model. SMV has rich finite data types, such as Booleans, scalars and fixed arrays, to describe the states; and therefore SMV shields away the implementation complexity caused by using machine friendly Boolean variables to describe the states. The assignment statement of SMV can be non-deterministic, assigning a variable any value from a set of values; or a variable may not be assigned a value at all. The system will try every possible value for that variable in the process of generating possible next states. SMV supports a module concept, which is crucial for any non trivial implementation of computer systems. Modules of SMV allow whole system to be broken into functional and abstract pieces to conquer increasing complexity of system implementation. Like C or Java, the "main" module is the starting 
point of running a program.

SMV system also has a language to describe specifications. It can specify CTL and LTL formulae in a concise syntax. OBDD based symbolic model checking algorithms are used internally to determine the satisfaction of specifications expressed in CTL or LTL.

We give a simple example to help readers understand SMV programs and our implementation described later. We also describe some key features uncommon in an ordinary programming language, but details will be explained in context of our implementations. See the following example counting from 000 to 111 [P183-184, 10].

module main()

\{

bito : counter_cell(1);

bit1 : counter_cell(bito.carry_out);

bit2 : counter_cell(bit1.carry_out);

S1 : SPECAGAF (bit2.carry_out);

\}

The "main" module is the starting point like C, Java language. The " : " is a delimiter token. SMV uses a module to call a sub-program as in other programming languages. SMV also uses a module to define a structure instead of inventing a class or struct type like the C language. The variable bito represents a group of variables defined in module counter_cello. It consists of the variable carry_out since the next statement use the variable bit0.carry_out as an input parameter.

The last statement specifies a property in CTL, which is true for the system. SMV will 
verify it, or give a counter example when an error happens. The keyword "SPEC" denotes that the statement is a property. The statement, $A G A F$ (bit2.carry_out), is the property specified in CTL. It means that the system will evolve eventually to reach a state, where the variable bit2.carry_out is true, with all paths from any states.

The following module is called by the above main program.

module counter_cell(carry_in)

\{

input carry_in : boolean;

carry_out : boolean;

value : boolean;

init(value) $:=0$

next $($ value $):=($ value + carry_in $) \bmod 2$

carry_out :=value \& carry_in;

\}

In the module, all variables are simple Boolean variables. In SMV, a Boolean variable have two values: 1 (true), 0 (false). It is treated as same as an integer with these two possible values. So we can apply arithmetic operators to Boolean variables directly. The characters " $:="$ is an assignment operator to distinguish it from the equal operator "=". The characters " $\&$ " and "|" are the "and" and "or" operations of logic, respectively. The operator "mod" returns remainder after dividing by 2.

We model a complete system by defining the initial state and how to get the next state from the current state. The keyword "init" and "next" are for these purposes. In the case where we have same formulae for computing values of some variables in the initial states 
and the next states; we do not need both keywords.

The module counter_cell(carry_in) basically says:

- If carry_in is true (I), the next state of the variable value is opposite to the current value; the next state of the temporary variable carry_out is same as the new value of the variable value.

- If carry_in is false (0), the next state of the variable value is same as the current value; the next state of the temporary variable carry_out is false $(0)$

The state transition system generated by the above main program is showed in the following figure:

\begin{tabular}{|llllllllll|}
\hline \multicolumn{1}{c}{ state } & 0 & 1 & 2 & 3 & 4 & 5 & 6 & 7 & 8 \\
Bit0.carry_in & 1 & 1 & 1 & 1 & 1 & 1 & 1 & 1 & 1 \\
Bit0.value & 0 & 1 & 0 & 1 & 0 & 1 & 0 & 1 & 0 \\
Bit0.carry_out & 0 & 1 & 0 & 1 & 0 & 1 & 0 & 1 & 0 \\
Bit1.carry_in from Bit0.carry_out & 0 & 0 & 1 & 0 & 1 & 0 & 1 & 0 & 1 \\
Bit1.value & 0 & 0 & 1 & 1 & 0 & 0 & 1 & 1 & 0 \\
Bit1.carry_out & 0 & 0 & 1 & 0 & 0 & 0 & 1 & 0 & 0 \\
Bit2.carry_in from Bit1.carry_out & 0 & 0 & 0 & 1 & 0 & 0 & 0 & 1 & 0 \\
Bit2.value & 0 & 0 & 0 & 1 & 1 & 1 & 1 & 0 & 0 \\
Bit2.carry_out & 0 & 0 & 0 & 1 & 0 & 0 & 0 & 0 & 0 \\
\hline
\end{tabular}

Figure 3.1 A state transition system generated by a SMV program

In Figure 3.1, if we discard the temporary variable carry in, we would find the state 8 is same as the initial state 0 . Considering that the current value of the variable carry_in does not affect anything for the next state, the state transition system would cycle from state 0 to state 7. From Figure 3.1, the counter program repeatedly count from 000 through to 111 by the three bit counters, Bit2.value, Bit1.value, Bit1.value. 
In SMV, no variables can be assigned more than once. For example, code like $\{x:=y ; \quad \ldots x:=z ;\}$ is not allowed since $\mathrm{x}$ is assigned twice. If a variable has not been assigned, SMV will try all possible values for the variable. All variables have limited values. If a variable is assigned a value out of the bound of the variable, it is given any valid value. Consider the following a piece of code:

\{

tmp : array $1 . .10$ of boolean;

default $\{$ for $(i=2 ; i<=10 ; i=i+1) \operatorname{tmp}[i]=0 ;\}$

in $\{$ for $(i=2 ; i<1=10 ; i=i+1)$ if (cond $[i]) \operatorname{tmp}[i]=1 ;\}$

\section{\}}

The for statement, similar to $\mathrm{C}$, is a loop from $i=2$ to $i=10$. The default statement says that variables are assigned if they are not assigned in the in statement. For example, if cond [3] is true, tmp [3] will be 1 or true; if cond [4] is false, the variable tmp [4] has not been assigned in the in statement, so $\operatorname{tmp}[4]$ will be 0 or false by the default statement. However, $\operatorname{tmp}[1]$ is not assigned by either of the statements, it will be given both 0 and 1 by SMV.

Sometimes there is a need to assign some variables more than once. So SMV has a chain statement. The chain statement is exactly same as the for statement except that a variable may be assigned more than once in the chain statement. For instance:

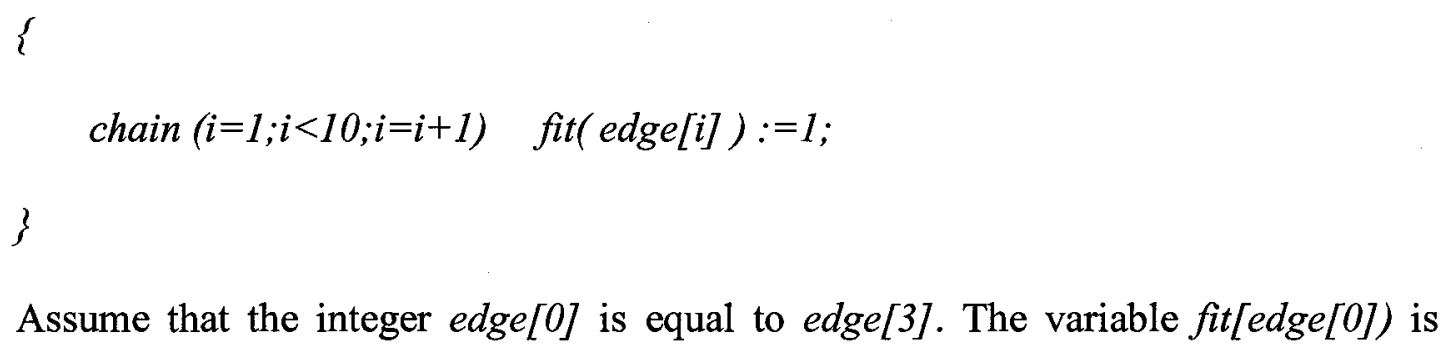

Assume that the integer edge[0] is equal to edge [3]. The variable fit [edge[0]) is 
assigned twice.

SMV allows operations on whole arrays.

$\{\quad$ tmp : array $1 . .10$ of boolean; $b 1$ : boolean; $b 2$ :boolean;

$b 1:=\& t m p ; \quad b 2:=\mid t m p ;$

\}

The operator "\&" and "|" are logical "and" and "or" operations respectively. The statement " $b 1:=\&$ tmp;" is equivalently expanded to :

$b 1:=\operatorname{tmp}[1] \& \operatorname{tmp}[2] \& \operatorname{tmp}[3] \& \ldots \& \operatorname{tmp}[8] \& \operatorname{tmp}[9] \& \operatorname{tmp}[10]$

SMV will expand the expression for us. Similar expansion is for "|".

SWV will try every possible value if a variable is not assigned. There is a statement that can assign multiple values to a variable. Look at the following piece of code:

$\{$ myvalue : array $1 . .100$ of $1 . .255$;

mycond : array 1..100 of Boolean;

myfinal : 1 .. 255;

......

myfinal $:=\{$ myvalue $[i]: i=1 . .100$, mycond $[i]\}$;

\}

From the last statement, the variable myfinal is assigned to anyone of a set of values obtained from myvalue[i], where the variable mycond[i] is true, and the iterative variable $i$ is from 1 to 100 .

With above knowledge, it is easy to understand our SMV program. 


\section{Chapter 4}

\section{Model data flow over the Internet using SMV}

Based on the ideas outlined in the above section, in this section we will model data packet flow over a network under firewall restrictions by using the SMV system.

I will use a very simple example to demonstrate my solution. The core program of the model is very concise, around two pages long. It does not change when the complexity of network topology and firewall configuration increases. The other parts in the program are data taken directly from network topology and firewall rules. The simple example is adapted from a personal firewall example in the book [4]. We duplicate the rule set for three personal computers. The actual firewall rule sets will be presented in the implementation. The simplicity of the example should give readers good understanding of the basic idea of our implementation.

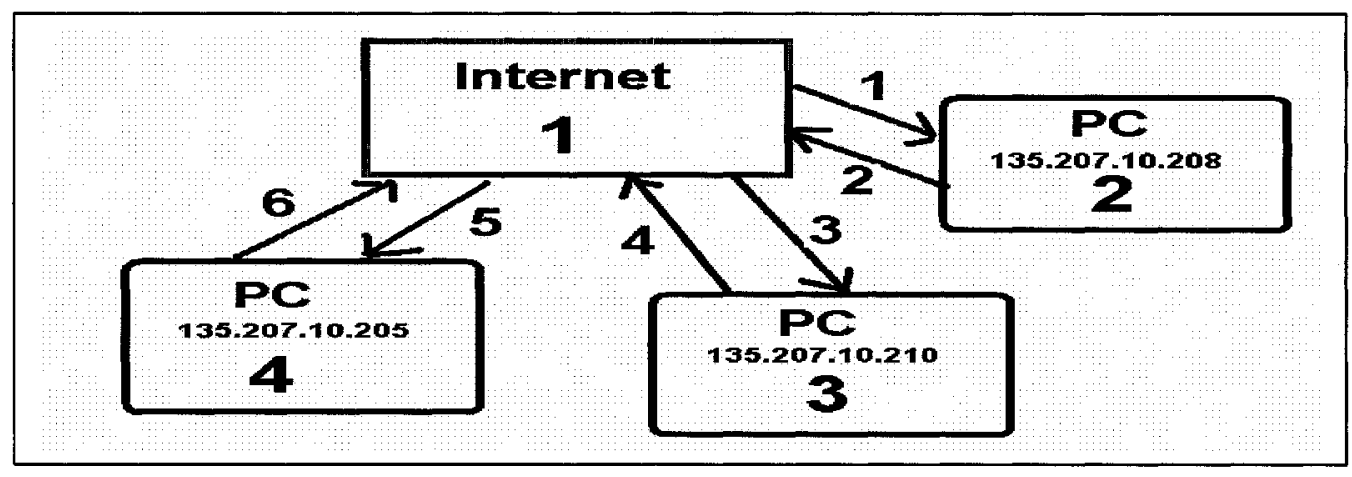

Figure 4.1 A simple example of multi personal firewalls 
In Figure 4.1 there are four zones. We will call a zone a position. Each position consists of a group of hosts or IP addresses. In the particular example, three of the positions only have one IP address each. However, we can treat a group of IP addresses in a same way as a single IP address using the mask representation discussed earlier. Generally, any position may contain a set of hosts (with IP address mask representations) and the final query may relate to subsets of these sets. Both may be represented by Boolean formulae. There are six directed edges to show where a packet can go from one position to another along that direction under the restriction of the attached firewall rule.

In the system the static part is the topology of networks, IP addresses dispatched in the zones and service port numbers in a single computer or IP address. The static part will tell the starting position of a packet based on its IP address allocation; similarly for the destination position of the packet. The dynamic part is the movement of the packet from one position to another position during its journey from source to destination. The packet may be blocked under firewall restrictions and not be able to reach its destination position. The dynamic packet flow can be regarded as a state transition system when a packet in a particular position represents a state.

A brief introduction on the main modules of the program will give readers an overview of the program and the SMV language. Then the program will be explained in detail in three parts:

- The data structure used to represent positions, packets, firewall rules and IP addresses allocated to each position (zone). The position and packet space represent the state space. The other data structures are for the purposes of implementation. 
- The modules to calculate the source and destination positions of a packet. This calculation decides the initial state or the initial position of a packet. It also tells the destination positions of packets. When spoofing attacks are considered, the initial state may be any position firewall system administrators want to set.

- The modules to decide the next position of a packet under the network topology and firewall restrictions from current position. If the packet has reached its destination or is blocked by firewalls, keep the current position as the next position.

The following code in the dataflow module for modeling a packet flow as a state transition is copied here. It will show the major components briefly. The complete code is in Appendix 2:

module dataflow ${ }^{\prime *}$ the module models the packet flow */

\{

thisPacket : packet();

/* structures for packets, no assignments for the initial state. SMV will try the whole packet space for us */

posi : 1..POSITION; /* the variable is for a packet in which position currently */ startposi : 1..POSITION;

$I^{*}$ startposi records the starting position when spoofing attacks are considered */ papo : packposi(thisPacket); /* calculation of source and destination position based on IP address allocation information*/

/*initial state */

init(posi) :=papo.sourposi; /* no initial if spoofing attack */ 


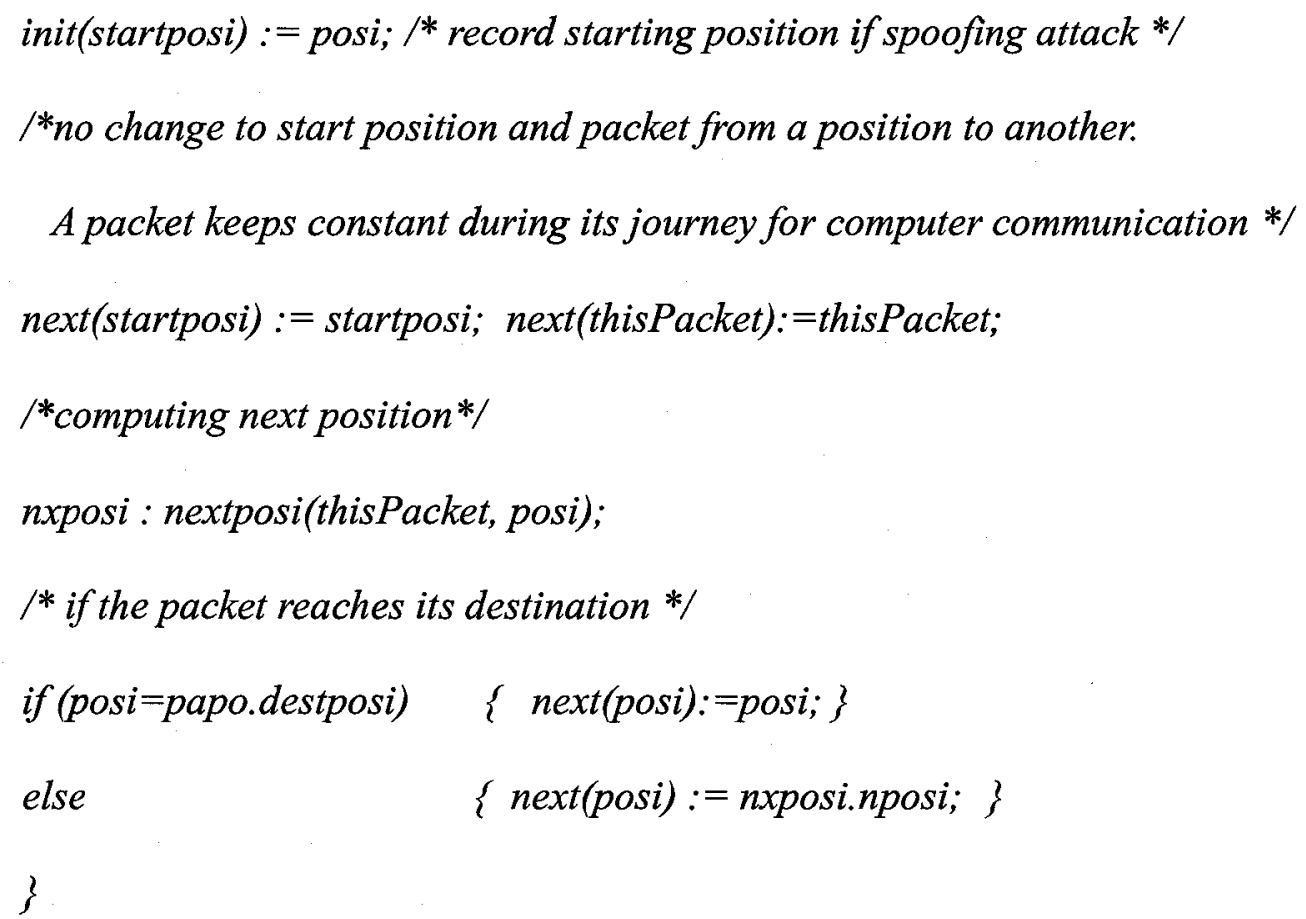

The dataflow module is to model the data flow over the Internet as a state transition system. The explanations are following:

- The first few statements are to define data for the system. The statement $\{$ thisPacket : packet()\} makes an instance of the module packet $($ to define a structure variable for a particular packet. There is no initial assignment statement for a packet, so SMV will try all possible values to assign as the initial state of the particular packet in modeling the state transition system. This is the same as trying all packets in the whole IP packet space.

- The statements $\{$ posi : 1..POSITION; startposi : 1..POSITION; $\}$ define variables for the network positions or zones. The "POSITION;" is a defined constant similar to constants used in C language. "posi : 1..POSITION" means that the variable posi can vary from 1 to POSITION. The posi variable represents which position a particular packet is in during its journey from source to destination. The 
starting position of a particular packet can be calculated from its source IP address. However, a packet may not begin to travel from its true source IP address or position when an IP address spoofing attack is considered. So the startposi variable is needed to record which position a particular packet starts at.

- In the statement \{papo : packposi(thisPacket); $\}$, the module packposi() calculates the source and destination position from the source and destination IP address of the packet, thisPacket. The parameter thisPacket gives the module packposi the input.

- The statement $\{$ init(posi) $:=$ papo.sourposi; $\}$ says that the packet will start its journey from the source address specified by the packet. The absence of this assignment would deal with spoofing attacks. The statement $\{$ init(startposi) $:=$ posi; $\}$ uses the variable startposi to record initial position of the packet. The variable startposi is not needed if we do not deal with spoof attacks.

- The packet keeps constant when it tries to flow from its source address machine to its destination. So we have statements:

$$
\{\text { next(startposi) := startposi; next(thisPacket):=thisPacket; }\}
$$

- The central task of this program is to decide if a packet can flow from a current position to another position under the restriction of firewall configurations. This is done by setting the next state of the variable posi. The 'if' statement decides the next state of variable posi in the module. It says that packet would stop if it has reached its destination position. The statement $\{$ ext $($ posi $):=p o s i ;\}$ is for this purpose. Otherwise, the statements \{nxposi : nextposi(thisPacket, posi); \} computes the next position and the statement $\{$ next(posi):=nxposi.nposi; $\}$ gets it. 
The module nextposi() takes the packet and current position as the inputs and checks appropriate firewall rules to get all possible next positions from its current position under the restriction of firewall configurations. There may be several positions a packet could move into under the firewall restrictions. If all next connected positions are blocked by firewall rules, the current position does not change.

The next section gives more detail on how the system evolves from a state to another state when a packet travels through networks under firewall restrictions.

\subsection{Data structure}

All actual data in this section except specified is copied from the simple example, whose architecture is in Figure 4.1 and it will be discussed in later chapter 5 . The size of data may differ in another example. Its structure stays same.

The data structure of packets is defined in the module packet(). module packet()

\{

prot:0..MAXPROTOCOL;

source, dest : array $1 . .4$ of $0 . .255$;

port : 0..MAXPORT;

\}

In an actual packet there are source and destination port number but they are usually 
same numbers. I use one variable port to model both ports only for simplicity. Also, we omit the flag bit which may be used for TCP connections for the same reason. Adding both these fields would not significantly affect the running time of the program. So I simplify packet model in the thesis.

The data for the network topology, i.e. the graph model is defined in the module. module topo()

$\left\{{ }^{*}\right.$ topo structutre,EDGE $=($ currp, nextp $)$, a directed edge $* /$

cposi,nposi : array 1..MAXEDGE of 1..POSITION;

/*ps-an array of Boolean variables. ps[i] will be true iff a packet can pass

the edge $i$ under the firewall restrictions */

ps : array 1..MAXEDGE of boolean;

/* the index of the arrays is the label number of edges in the graph model, the values of arrays are respectively out and in position (zone) a directed edge points to */ cposi: $=[1,2,1,3,1,4] ; / / 6$ edges, the starting vertex number of edges nposi: $=[2,1,3,1,4,1] ; / / 6$ edges, the ending vertex number of edges * each edge attaches a firewall rule set; the index of the arrays is the number of a edge */ \}

The data for the variables cposi and nposi is from the example specified in later chapter 5. For now we only need to know that the directed edge $i$ is from position cposi[i] to the position nposi $[i]$. In different applications, the data, which includes numbers of edges and position, is different. But the structure stays same. Refer to Appendix 3 for a different example.

The data structure of a firewall rule is defined in the following module arule 0 . 
module arule()

$\{/ *$ all firewall rules are represented in one array of arule() */

edge : 1..MAXEDGERULE; / each edge in the graph has a number */

permit : boolean; /* the action is taken if a packet matches it */

prot : 0..MAXPROTOCOL; /* protocol number*/

/* source IP address, mask and destination IP address and mask*/

$s, s m, d, d m$ : array $1 . .4$ of $0 . .255$;

/* minimal and maximal port number representing a range of port numbers */

minp, $\operatorname{maxp}: 0 . . M A X P O R T$;

\}

The first variable edge indicates which edge the rule is attached to in our graph model. An actual rule may work for both direction between two zones or positions; however, the edge in the graph model is one-way. The second variable permit is for the action: pass or drop when a packet matches the rule. Again there are more actions in a firewall product. The rest of the rule data structure is the criteria for what kinds of packets match the rule. We can construct a list of rules for the entire firewall rule sets. The list in module rulesets $($ is “rules : array 1..MAXRULELIST of arule0;". An example of representing the rule set is: module rulesets()

\{

rules : array 1..MAXRULELIST of arule();

* rule1--1-2*/

t11 : setarule(rules[1], 1,1, TCP, 0,0,0,0, 0,0,0,0, 135,207,10,208, 255,255,255,255, 0,MAXPORT); 
t12: setarule(rules[2], 1,1, UDP, 0,0,0,0, 0,0,0,0, 135,207,10,208, 255,255,255,255, 53,53);

t31: setarule(rules[13],3,1, TCP, 0,0,0,0, 0,0,0,0, 135,207,10,210, 255,255,255,255, $0, M A X P O R T)$

$\cdots$

\}

The setarule() module puts a rule into the rule data structure arule() and is straightforward. The first two rules are attached to edge 1 , the third rule is attached to edge 3 in the above example.

The data structure for IP address allocation to positions or zones is defined in the following module.

module aposi()

$\left\{{ }^{*}\right.$ A group of IP addresses are allocated to this position or zone */

posi : 1..POSITION;

/* IP address with mask to represent a group of IP addresses in one particular position */ addr, mask : array $1 . .4$ of $0 . .255$

\}

The program uses masked IP address representation to decide what IP addresses are allocated to the position that the variable posi denotes. A position may have several representations of this form in a complicated case. The list of positions is defined in module positions() \{ posi : array 1..MPOSIRULE of aposi();...\}. An example is as following: 
module positions()

\{

posi : array 1..MPOSIRULE of aposiO;

t1 : setaposi(zone[1],2,135,207,10,208, 255,255,255,255);

t2 : setaposi(zone[2],3,135,207,10,210, 255,255,255,255);

t3 : setaposi(zone[3],4,135,207,10,205, 255,255,255,255);

\}

The module setaposi() sets IP address allocations in the above data structure. The three statements set IP address allocations in position 2, 3, and 4 respectively. The other unspecified IP addresses in the module belong to the position 1 , usually the Internet (the public Internet in our example).

We may also model routing. From the point of security, we want to prevent certain packets from reaching their destinations. If there exists a path not allowed by a router and unwanted packets are still blocked along the path by firewalls, then the system is secure. Thus, for simplicity, we do not model routing in this example. For the larger example in Chapter 6, we can model routing as follows.

The data structures for routing are a default path, which a packet goes to if no next hop is found from the routing table in a particular router or position, and the next hop information from the routers:

module defaultroutetable0

\{ dedge : array 1..POSITION of 1..MAXEDGE;

dedge $:=[11,2,4,6,8,10,12,14,16]$;

\} 
The module defaultroutetable() gives the default next hop (edge) for a packet in a specific position during its journey.

The following module aroutpath() defines a next hop for a packet in a specific position or router.

module aroutpath0

\{

posi : 1..POSITION;

edge: 1..MAXEDGE;

addr, mask : array $1 . .4$ of $0 . .255$;

\}

The following module actually provides routing information except that specified in a default path. The module setaroutpath() sets data for the routing table. The constant MROUTETABLE is the size of the table. Some detail is in Appendix 3.

module routetable()

\{rtable : array 1..MROUTETABLE of aroutpath0;

r1 : setaroutpath(rtable [1], 1, 1, 234,217,225,0, 255,255,255,224);

r5 : setaroutpath(rtable[5], 4, 9, 234,217,5,132, 255,255,255,252);

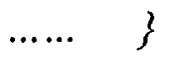

We are now ready to describe how the initial state and next state are calculated. 


\subsection{The initial state}

Recall that we use a packet and a position to represent a state. The initial value of a packet does not need to be set. SMV will try all possible values and therefore the whole IP packet space is tested. We can determine the source and destination position using module packposi(thisPacket). The initial position is papo.sourposi, where papo is an instance of module packposi(thisPacket). The module takes a specific packet as the input and obtains its source and destination position. The module is as follows.

module packposi(pack)

$\left\{{ }^{*}\right.$ this packet actual source and destination position $\left.*\right\}$

input pack : packet(); /* the specific packet */

sourposi : 1..POSITION;

destposi : 1..POSITION;

zone : zones( $) ; / *$ the data for allocated IP addresses */

for $(i=1 ; i<=$ MPOSIRULE; $i=i+1)$

s[i] : IPgroupmask(pack.source, zone.zone[i].addr, zone.zone[i].mask);

/* compute if the packet source IP in the ith zone (position) */

default $\{$ sourposi: $=1 ;\}$

in \{

$\operatorname{chain}(i=1 ; i<=$ MPOSIRULE; $i=i+1)$ if(s[i].fit=1) sourposi := zone.zone[i].posi;

\} 


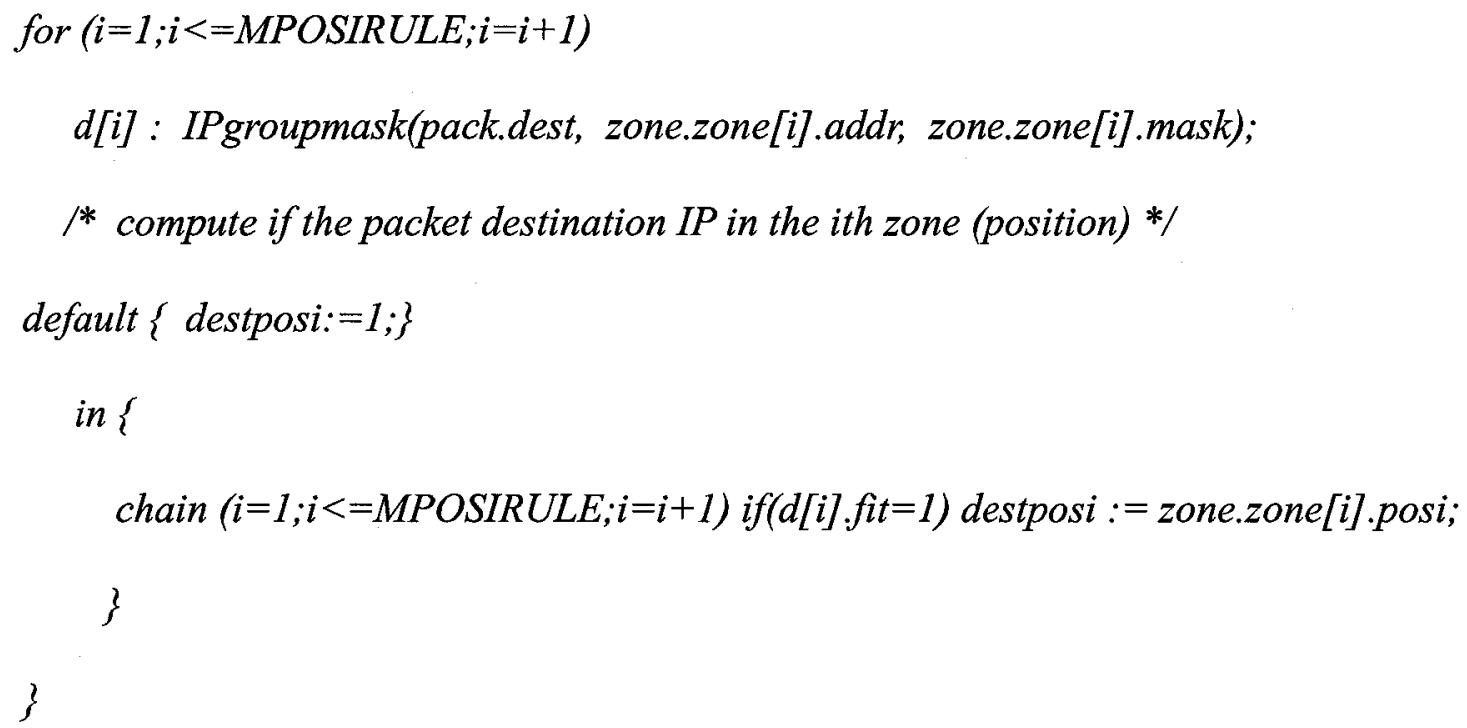

The module IPgroupmask() decide if a specific IP address fits in a group of IP addresses with the masked IP address representation.

module IPgroupmask(paddr,zaddr,zmask)

$\left\{/^{*}\right.$ return whether an IP address belongs to the position or a group of IP addresses,

which is expressed in IP address with mask representation */

input paddr, zaddr, zmask : array $1 . .4$ of 0..255; /*IP address and mask are 32-bits long*/ fit : boolean;

/* use 4 integers to represent the 32-bits */

for $(i=1 ; i<5 ; i=i+1)$

/* The module IPgroupmaskfunc is same as the module fitaposi except its bits are shorter*/ f[i] : IPgroupmaskfunc (paddr[i], zaddr[i], zmask[i] );

/* a match needs every bit match of the 32 bits */

$f i t:=(f[1] . \operatorname{tr} \& f[2] . \operatorname{tr} \& f[3] . \operatorname{tr} \& f[4] . t r)$;

\}

The module IPgroupmask() for 32-bit IP address is decomposed into using the 8-bit 
module IPgroupmaskfunc() with the same function.

module IPgroupmaskfunc(pa,za,zm)

\{

/* decide whether a IP address belongs to a group of addresses with mask representation. */ input pa, za, zm : array $1 . .8$ of boolean;

temp : array $1 . .8$ of boolean;

tr : boolean; /*fit in or not*/

for $(i=1 ; i<9 ; i=i+1)$

/* bit match: if mask bit is true, packet bit pa must be equal to rule bit za;

otherwise pa is free, it can be true or false. */

$\operatorname{temp}[i]:=((\sim z m[i]) \mid(p a[i]=z a[i])) ;$

* match: all bit must be matched by the and or conjunction Boolean operation */

tr $:=\&$ temp;

\} 


\subsection{The next state}

After the initial state is set, we need know how to compute the next state is from the current state. A packet stays constant during its journey, so the value of its next state is equal to the one of its current state. The only needed change is for the position. Let us revisit the part in the dataflow module:

module dataflow()

$\{/ *$ next state calculation */

$I^{*}$ no change to start position and packet from a position to another.

A packet keeps constant during its journey from source to destination */

next(startposi) := startposi; next(thisPacket):=thisPacket;

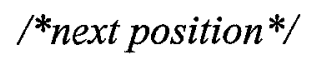

/* the module nextposi decides where the packet can go under firewall restriction */

nxposi : nextposi(thisPacket,posi) ;

/* if the packet reaches its destination host, default */

default $\{$ nextposi : $=$ posi; $\}$

in $\{$ if ( $\sim(p o s i=$ papo.destposi $))$ nextposi $:=n x p o s i . n p o s i ;\}$

next(posi) := nextposi;

\}

If a packet reaches its destination position, it stops travelling. Otherwise it continues to travel to positions permitted by relevant firewall rules, and routers. The module nextposi() 
calculates permitted next positions under the firewall restrictions. There may be several options of next positions (zones). This reflects that a packet may have several paths during its journey. The routing module is optional. When it is used, the next position is the next hop permitted by rules. This usually happens at a router.

module nextposi(pack,currposi)

$\{/ *$ packet and current position : currposi $* /$

input pack : packet(); input currposi : 1..POSITION;

* the next permitted position */

nposi : 1..POSITION;

/* the walls module returns whether a packet is permitted to move into its next position from the current position by a rule set attached to an edge whose starting vertex is the current position. */

wl : walls(pack); /* The firewalls decide if a packet can pass a wall */

$r t$ : routing(pack.dest); /* next hop (edge) based in routing information */

tmp : array 1..MAXEDGE of boolean; /* temp variables */

/ topo structure, Edge = (currposi,nextposi), a directed edge */

for (iedge $=1 ;$ iedge $<=M A X E D G E ;$ iedge $=i e d g e+1$ )

tmp[iedge]:=(wl.tp.ps[iedge] \& rt.path[i] \& wl.tp.cposi[iedge]=currposi);

/* The above assignment statements say that if the starting vertex of the edge number iedge is not equal to the current position, then the edge is not relevant to the decision of the next hop for the packet in the current postion. So tmp[iedge] is false. If one of tmp array is true then the packet can pass an iedge interface into its nextposi */ 
if $(\mid t m p)$

$\{$ nposi $:=\{$ wl.tp.nposi[iedge] : iedge=1..MAXEDGE, tmp[iedge]\}; $\}$

else

$\{$ nposi :=currposi;\} $/ *$ no permitted next position, keep it as current*/

\}

The character "|" denotes "or" operation of Boolean variables. The variable tmp is a vector of Boolean variables. The expression $(\mid t m p)$ is abbreviated as follows:

$$
\operatorname{tmp}[1]|\operatorname{tmp}[2]| t m p[3]|\ldots| \operatorname{tmp}[M A X E D G E-1] \mid \operatorname{tmp}[M A X E D G E]
$$

We will now explain how the walls() module works. The module walls() decides if the input packet is permitted by the firewall rules.

module walls(pack)

\{input pack : packet0;

tp : topo();

/* part one decides, for each rule set, whether a packet matches a rule of the set. */

rlist : rulesets();

for $(i=1 ; i<=$ MAXRULELIST; $i=i+1)$

f[i] : fitarule(pack,rlist.rules[i]);

/* part two decides, for each edge, whether a packet can pass an edge based on the attached ordered firewall rule set */

default \{

for $($ iedge $=1 ;$ iedge $<=M A X E D G E R U L E ;$ iedge $=i e d g e+1)$

tp.ps[iedge]:=0; /*if no rule fits, denial $*$ /

\} 
in \{

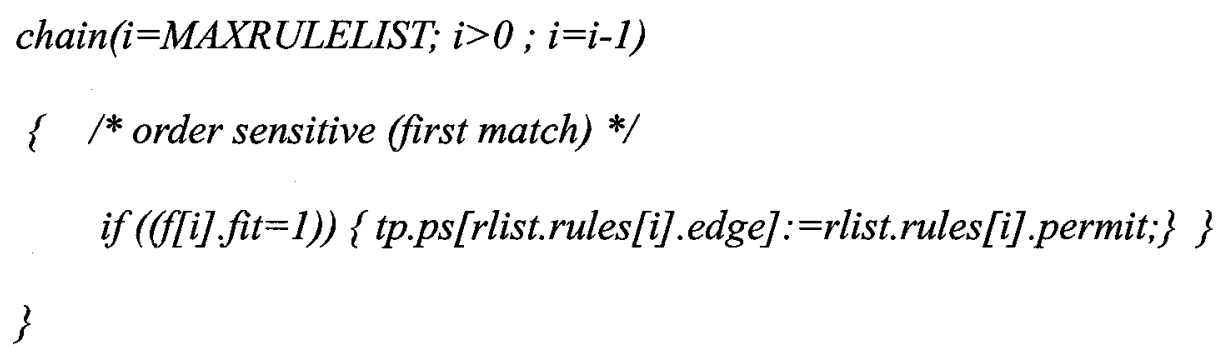

$/ * p s=$ true--a packet can pass the firewall pointed by the edge of iedge */

/* Take the action if the packet matches a rule attached to an edge when the packet goes along the directed edge. If several rules attached to an edge are matched, then the action of the first matched rule will be taken. The chain statement is used to allow repeated assignment when a packet satisfies multiple rules. The chain statement is same as the for loop statement except that it allows repeated assignment to a variable. The calling module walls() will consider whether the edge is being touched, namely if the starting position of the edge is the current position */

\section{if (MAXEDGE $>M A X E D G E R U L E)$}

$\left\{/^{*}\right.$ in case no rule for some edges or no firewall in place to block. */ for (iedge $=M A X E D G E ;$ iedge $>M A X E D G E R U L E ;$ iedge=iedge-1) tp.ps[iedge] :=1 ;

We will now discuss the module fitarule 0 briefly, which tells if a packet matches a rule:

module fitarule(pack, ar)

\{

/* a packet fits into a rule */

input pack : packet0; /* a packet */ 
input ar : arule(); /* a rule */

fit : boolean;/* record whether the packet matches the rule */

/* using 4 integers to represent 32 bit IP and mask scheme */

for $(i=1 ; i<5 ; i=i+1)$

$f[i]$ : fin(pack.source[i],pack.dest [i],ar.s[i],ar.sm[i],ar.d[i],ar.dm[i]);

/* all bits must match if a packet is considered as a match to a rule */

$f i t:=(f[1] . t r \& f[2] . t r \& f[3] . t r \& f[4] . t r)$

$\&(($ ar.prot $=$ ANYPROTOCOL $) \mid($ pack.prot $=$ ar.prot $))$

$\&($ pack.port $<=$ ar.maxp $) \&($ pack.port $>=$ ar.minp $) ;$

\}

The module fin 0 calculates if the IP addresses of a packet match a rule, which specifies a group of IP addresses using an IP address with a mask representation for 8 bit integers. The module is quite simple as follows.

module fin(so,de,s,sm,d,dm)

$\{/ *$ source and dest addresses fit under masks */

input so,de,s,sm,d,dm : array $1 . .8$ of boolean;

temp : array $1 . .8$ of boolean;

tr : boolean;

/* if the mask bit is true, then the corresponding bit of the packet must be equal to the corresponding bit of the rule. Otherwise, the bit of the packet is ignored, namely it can be either true (1) or false (0). This principle applies to both IP source and destination address of the packet. See the following statement. */ 
for $(i=1 ; i<9 ; i=i+1)$

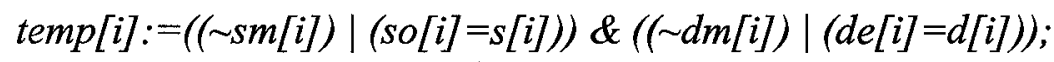

$/ *$ all bits must match if we regard that the packet match the rule */

tr : = \& temp;

\}

The expression (\&temp) is similar to the statement (| temp) except "\&" denoting "and" operation of Boolean variables.

The following module routing (destaddr) is for routing information. Based on the destination address of a packet, it gives a next hop when the packet is in different positions or routers.

module routing(destaddr)

\{

input destaddr : array $1 . .4$ of $0 . .255$;

path array 1..MAXEDGE of boolean;

thelook : lookup(destaddr);

dtable $\quad$ : defaultroutetable();

default \{

${ }^{*}$ if there is no routing information for a particular path (edge), no go. This case does not happen if the routing information is correct and complete*/ for $(i=1 ; i<=M A X E D G E ; i=i+1)$ path $[i]:=0$;

\}

in 


\section{\{}

default $\{$ chain $(i=1 ; i<=$ POSITION; $i=i+1)$

$\{$ if (thelook.defaultpath [i] =1) path[dtable.dedge $[i]]:=1 ;\}$

/* if no matching to the routing table, go to the default path for the packet in the position */

\} in \{

$\operatorname{chain}(i=1 ; i<=$ MROUTETABLE; $i=i+1)$

$\{$ if (thelook.look[i].fit=1) path[thelook.rt.rtable[i].edge] :=1; $\}$

\}

\}

\}

The module lookup(destaddr) decides whether the destination address of a packet in a position is fit in a rule of the lookup table, or the packet goes the default next hop.

module lookup(destaddr)

$\{/ *$ this packet actual source and destination position */

input destaddr : array $1 . .4$ of $0 . .255$;

$r t$ : routetable(); /* lookup table*/

* flag for default path, if a packet does not match any rule in the routing table in a position, the packet should go to the default path*/

defaultpath : array 1..POSITION of boolean;

* flag a rule as fit for the packet*/

for $(i=1 ; i<=$ MROUTETABLE; $i=i+1)$

look[i]:IPgroupmask(destaddr, rt.rtable[i].addr, rt.rtable[i].mask);

/* if the packet in a certain position has no fit rules, flag it as a default path */ 


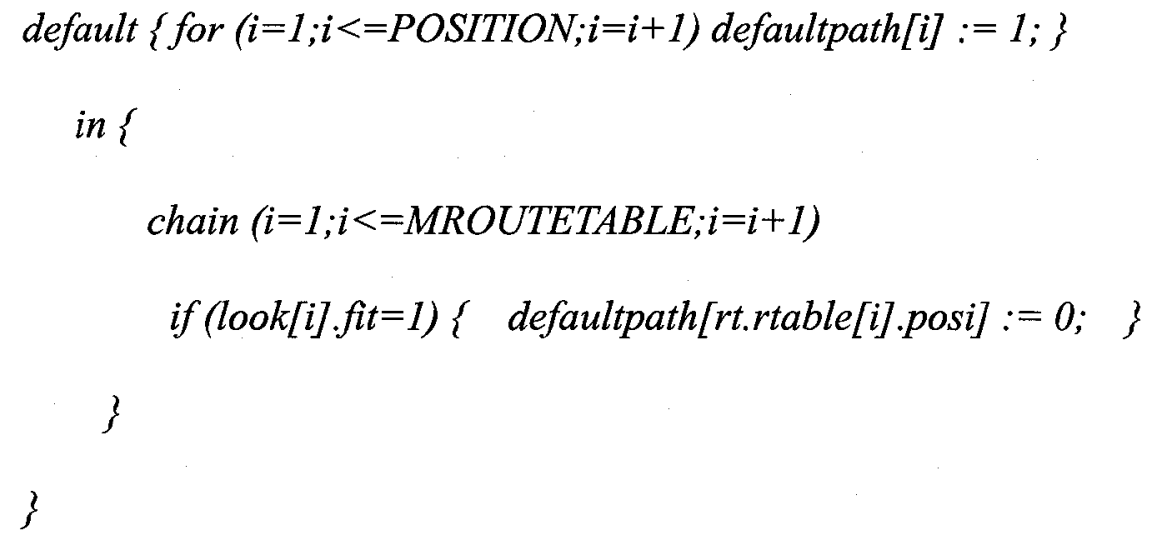

We have now explained all the major modules related to the graph model of networks under firewall restrictions. 


\section{Chapter 5}

\section{How to answer queries about firewall configurations}

After modelling the data packet flow under firewall restrictions as a state transition system or its equivalent Boolean formula, we are ready to answer queries about firewall configurations. As discussed in the introduction, we may have many specific questions under different circumstances. These questions may eventually lead into what packets should or should not reach certain positions (zones) and we want to confirm that firewall configurations achieve these goals exactly. They can be in form of a query to verify correctness of firewall configurations or to reveal weakness. With SMV, there are two major ways to deal with the query:

1. Specifying the query as a property using CTL or LTL. SMV will verify its correctness or give counter examples. This is basically for those queries having specifics about what data should or should not reach certain zones. This capability covers most questions able to be answered by the three previous works discusses earlier.

2. Interpreting the query as a Boolean formula. Run its conjunction operation with the Boolean formula of the model or the state transition system, which can be provided as an OBDD in the SMV commercial version, to get the result as a Boolean formula. Then interpret and present results in user-friendly manner as in the Hazelhurst paper $[7]$. 
Let us first discuss for the first solution using the simple example given in the previous section. The following is copied from the main module of my implementation. module main 0

\{

df : dataflow();

/ The following temporary variables are just for intermediate expression */ bsource, bdest2, bdest, btcp : boolean;

bsource: $=($ df.thisPacket.source $=[135,207,10,208]) ; / *$ position $2 * /$

bdest $2:=($ thisPacket.dest $=[135,207,10,208]) ; / *$ position $2 * /$

bdest $:=($ thisPacket.dest $=[135,207,10,210]) ; / *$ position $3 * /$

btcp: $=($ df.thisPacket.prot $=T C P)$;

/** Two Specified property **/

tcp : SPEC (btcp \& bsource) -> $A G A F \sim(p o s i=2)$;

_tcp23 : SPEC (btcp \& bsource \& bdest) -> AXAXAG (posi=3);

\}

Please refer Figure 4.1 as the network architecture. The relevant rule set is in the code of Appendix 1. For the example, we test two properties using CTL specifications.

The first CTL specification says that TCP packets from a computer of the position 2 are not blocked by firewalls and can leave the position. In the specification, the formulae (btcp \& bsource) is a condition to states what packets the query is for. The part of the specification, $A G A F \sim(p o s i=2)$, means that the system will reach states, where the variable posi is not 2 , with all possible paths starting from all states, where the above condition ( btcp \& bsource) is true. This specification is not true. SMV exactly gives us a counter 
example that a packet with its destination address in position 2 will never leave the position 2 since the packet stops moving when it reaches its destination. We need to change the above specification excluding the packets into:

SPEC (btcp \& bsource \& bdest2) -> AGAF (posi=2);

The expression, $\sim$ bdest 2 , says the packet destination address is not in position 2 . SMV will verify the new specification as correct. Note our firewalls pass the above packets with any port numbers. In practice, firewalls usually permit packets with some port numbers and block packets with other port numbers.

The second CTL specification confirms TCP packets from a computer of position 2 can reach their destination, position 3. The condition, ( btcp \& bsource \& bdest), specifies those packets. The CTL formulae, $A X A X A G$ (posi=3), says that all the states have posi=3 after at least 2 transitions from an initial state

If the specified properties fail, SMV will give a counter example to show what goes wrong. Then appropriate actions can be taken to fix the problem.

Additional power of the tool will come with the second solution. Recall we have a Boolean formula to represent a state transition system that model data packet flow over a network under firewall configurations. SMV also provides a Boolean formula that represents the set of all states that can be reached by a sequence of transitions from an initial state. We call this formula $S(P, Z)$, where $P$ and $Z$ represent variables for packet data and zone, respectively.

We now can answer queries by taking advantage of the Boolean formula. We discuss how to answer queries in term of Boolean operations, although the actual actions would be performed on their OBDDs. 
A query about what packets can or cannot reach certain zones can be expressed as a Boolean formula. Suppose it is:

$$
Q(P, Z)
$$

The query result will be from conjunction operations of the reachable and query Boolean formulas:

$$
S(P, Z) \text { and } Q(P, Z)
$$

Let us try several typical queries in more detail to understand the procedure.

Query 1: "Does the packet reach its destination, zone 7?"

Assume that we have a Boolean formula to represent the packet:

$$
Q(P)
$$

Then we can get the query result as the following formula:

$$
R(S, Z):=(S(P, Z) \text { and } Q(P) \text { and } Z=7)
$$

The Boolean value for the packet reaching its destination will make the formula true.

Query 2 "Is the packet blocked and not able to reach its destination, zone 7"

This query will be confirmed if the above formula is forever false.

Query 3: "what packets can reach zone 7?"

Firstly we can have a Boolean formula to represent packets with their destination IP addresses allocated into zone 7:

$$
Q(P)
$$

The results of the query will be in the Boolean formula by using conjunction operations:

$$
R(P, Z):=S(P, Z) \text { and } Q(P) \text { and }(Z=7)
$$

The result will tell you what computers can talk to the computers in zone 7 with what 
services and protocols.

Query 4: "What packets can reach their final destination?"

Based on the solution to the query 3 , we can obtain results by having a disjunction operation of all Boolean formula for all zones.

Query 5: "What happens if we remove a rule from a firewall"

With query 4, we have two Boolean formulae representing successful packets of before removing and after removing respectively. By running the $X O R$ operation on the two formulae, we get the final formula as a result. The result may say that a computer can talk with another computer with certain services only after a rule is removed, or vice verse. When any other change in firewall rules happens, our tool can tell what is the exact difference on the packet flow. This information could be quite useful and it is very difficult to get without the appropriate tool. If the formula is forever false, which means nothing changed, the rule is redundant and unnecessary.

We have not implemented this second approach to queries. An implementation would be straightforward, similar to what was done by Hazelhurst [7].

It is quite simple and straightforward to deal with spoofing attacks, where packets may come from a different zone than the zone in the source IP addresses of the packets. In the implementation, the only difference is to remove the assignment for the initial state of the position variable posi obtained from a packet source IP address and IP address allocation information. The system can then start from any position. Queries about spoofing attacks are similarly processed as the above queries. The only addition needed is to specify a initial position for the variable startposi in the Boolean formula for spoofing attacking packets. 


\section{Chapter 6}

\section{Performance of our approach}

So far we have described the theory and implementation of our tool (approach). In the section we will discuss its performance. During security analysis of firewall configurations, the tool should answer queries in a timely and reasonable manner although they are batch jobs.

In theory, the computational complexity may be exponential because the complexity of the underlying OBDD implementation of SMV is exponential. However, in practice OBDD technology is a major breakthrough because it actually does not grow fast as exponential [10] in many practical cases. Many successful SMV applications have demonstrated that.

The example, provided by Scott Hazelhurst [7], showed good performance. However, because we are modeling whole networks instead of just a single firewall, our state transition system will be much larger. Thus it is not clear that queries can be answered in a reasonable time using this technology in this new setting. We show that it is, in fact, possible to get useful results using our tool by demonstrating it on a moderately-sized, but realistic, example network.

The example is adapted from actual firewall configurations of Carleton University's School of Computer Science and Security Lab. We implemented all the firewall rules 
except those not related to packet filtering. I duplicate the firewalls of the school and lab, and modify some rules to make the example more complicated and interesting. All firewall rules for the example can refer to the code in Appendix 3.

The following picture shows the topology, IP allocation and firewalls of the example:

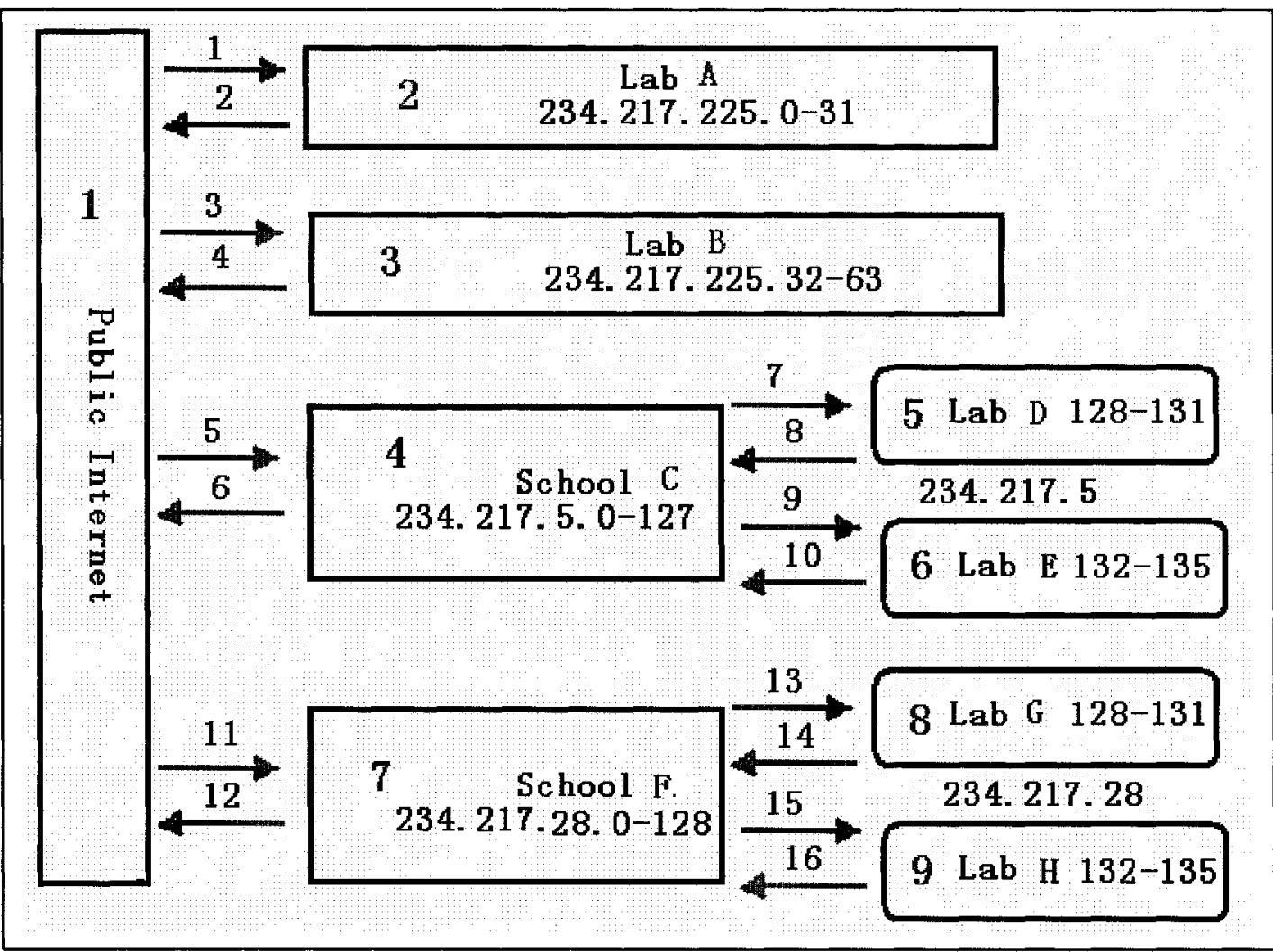

Figure 6.1 An example of multi-firewall configurations of an institution

The routing information refers to the code in Appendix 3. Without routing information, a packet, which has its source address lab $\mathrm{H}$ and destination address lab G, may go outside school F. This does not really matter when we care about if the packet can reach Lab G.

The following queries are specified in CTL. For details refer to Appendix 3. If the properties fail, SMV will give a counter example, which may reveal a weakness or error in firewall configurations: 
query1 : SPEC (btcp \& bpwww \& ( (bsipdomain)) \& bdestlabawww) -> EF (df.posi $=2)$;

The query basically says that any computers from the Internet can reach the web server in Lab A. SPEC is a keyword of SMV to specify a property in CTL. The variable btcp denotes whether packets are in a TCP connection. The variable $b p w w w$ is for web service. Its value is a port number for the web service. In our simplified model, we assume only one port number instead of the source and destination port number of an actual packet. The variable bsipdomain denotes packets with source IP address starting with 234.217. This means that packets come from the Internet. The variable bdestlabawww denotes that packets go to the web server of the Lab A, whose IP address is 234.217.225.13. The assertion $E F($ df.posi $=2$ ) means there exists a path along which the packets can reach position 2, Lab A, where the web server is located. For detail on these values, refer to Appendix 3. If routing information is included in the model, the $E F$ may be replaced by $A F$, which means that all paths will succeed. In this specific case, there may be no difference because there is only one meaningful path. However, we are only concerned whether a packet can reach its destination.

query2 : SPEC (btcp \& bpwww \& ( brestrictedlab) \& bdestlabawww $)->E F((d f . p o s i=$ 2));

The query says that computers from restricted Lab $\mathrm{E}$ or lab $\mathrm{H}$ can visit the web server in Lab A. The variable brestrictedlab represents packets coming from Lab E or Lab H.

query3 : SPEC (btcp \& df.startposi $=3 \&$ df.thisPacket.source[4]>48) -> AG (df.posi $=3$ );

Any TCP connection to outside Lab B from its some computers is blocked. The 
assertion $d$ f.startposi $=3$ says packets from Lab B. The source IP addresses are not represented explicitly. The assertion $d$ f.thisPacket.source[4] $>48$ means that packets must come from computers in Lab B with the last 1 byte of the 4-byte source IP address bigger than 48. In other words, their source IP addresses are from 234.217.225.49 to 234.217.225.64. The CTL formula $A G(d f . p o s i=3)$ means that all states always contain position 3.

query4 : SPEC (btcp \& bp631 \& bdestlabb631 \& df.startposi=4) -> EF (df.posi=3);

Any computers from school C (Position 4) can use the web service 631 in LAB B with TCP connection. The variable $b p 631$ is for web service 631 .

query5 : SPEC (btcp \& bp631\& bdestlabb631\& ((df.startposi=3) |(bsip5))) ->AG $\sim(d f . p o s i=3)$;

Except from School C, Lab D and Lab E, no computers can use the web service 631 in LAB B (position 3) with TCP connection.

query6 : SPEC (btcp \& bpwww \& ( (bsipdomain)) \& bdestcwww) -> EF (df.posi = 4);

All computers from the Internet can visit the web server in School C.

query7 : SPEC (bprintservice \& ( (bsipdomain)) \& bdestcprn) $->A G \sim($ df.posi $=4)$;

No computers from the Internet can use the printer server in School C. The variable bdestcprn denotes the printer server.

query8 : SPEC (btcp \& bpwww \& (bsip5) \& bdestdwww) -> EF (df.posi = 5); 
Computers from School C, Lab D, and Lab E can reach the web server in Lab D. The variable bsip5 denotes packets with source IP addresses starting at 234.217.5 query9 : SPEC (btcp \& bpwww \& (bsourf) \& bdestewww) ->AFAG (df.posi $=6)$; The computer with IP address 234.217 .28 .22 , decided by the variable bsourf, from School $\mathrm{F}$ is not allowed to visit the web server in restricted Lab $\mathrm{E}$ in position 6.

query10 : SPEC (df.startposi=6 \& ( bdip5)) -> AG ((df.posi=6));

Firewall 10 blocks any packets with destination addresses to outside School C, Lab D and Lab $\mathrm{E}$ if they are from the restricted Lab $\mathrm{E}$ (position 6).

The test environment is an average PC of the year 2001 with intel CPU, Redhat Linux. The total time for the above 10 queries is about 64 seconds and the time of each query is almost same. These are clearly reasonable times for offline firewall analysis. 


\section{Chapter 7}

\section{Conclusions and future work}

Our tool needs more work to be truly useful, for example:

1. A user-friendly interface is essential in practical use. Users should not be exposed to underlying techniques such as CTL, SMV, OBDD. Users should have friendly input and output in a form familiar to firewall administrators.

2. The tool should collect propriety firewall rules and translate into SMV code automatically. The tool should also translate user input into CTL, Boolean formulae or their operations, and vice versa.

3. The tool should incorporate something such as TCP flags, and other actions ignored in our model for simplicity.

Lots more may be needed as a useful product. However, the thesis gives the key ideas and implementation and shows that the approach is practical.

In the future, we should integrate the tool with other firewall products to let the market test its usefulness. Practice may present new problems and new needs for the tool. Certainly lots of case applications are necessary to claim usefulness of our tool, even if we cannot prove that the complexity with special structure is polynomial.

We also can optimize the OBDD structure and operations based on the special structure in the model of packet flow under firewall restrictions 
In a broad view, the use of access control becomes critical in information security and its problems become increasingly complicated with accelerating information applications. We might be able to use the ideas in the thesis to provide promising and quick solutions. 


\section{References}

[1] Stefan Axelsson. Intrusion detections systems: a taxonomy and survey. Technical Report 99-15, Department of Computer Engineering, Chalmers University of Technology, Sweden, March 2000.

[2] Yair Bartal, Alain Mayer, Kobbi Nissim, Avishai Wool. Firmato: a novel firewall management toolkit. In proceedings of 1999 IEEE symposium on security and privacy. P17-31.

[3] Chrisopher J. Calabres. A tool for building firewall router configuration. Computing Systems, 9(3):239-253, 1996 USENIX Association.

[4] W.R.Cheswick and S.M. Bellovin, Firewalls and Internet security: repelling the wily hacker, Addison-Wesley, 2003.

[5] Cisco PIX firewall user guide, V3.0. http://www.cisco.com/en/US/products/sw/ secursw/ps2120/products configuration guide book09186a0080485989.html, 2004.

[6] Pasi Eronen, Jukka Zitting, An expert system for analyzing firewall rules, In Proceedings of the 6th nordic workshop on secure IT systems (NordSec). P100-107, Copenhagen, Denmark, November 2001.

[7] Scott Hazelhurst. Algorithms for analysing firewall and router access lists. technical report TR-Wits-CS-1999-5, department of computer science, University of the Witwatersrand, South Africa, July 1999.

[8] Scott Hazelhurst, Adi Attar, and Raymond Sinnappan. Algorithms for improving the 
dependability of firewall and filter rule lists. In Proceedings of the international conference on dependable systems and networks (DSN 2000), pages 576-585, New York, June 2000. IEEE Computer Society Press.

[9] Kent Hundley and Gil Held. Cisco access list field guide. McGraw-Hill, March 2000.

[10] Michael Huth, Mark Ryan. Logic in computer science: modeling and reasoning about systems. Cambridge University Press 2002

[11] Alain Mayer, Avishai Wool, Elisha Ziskind, Fang: A firewall analysis engine. In proceedings of 2000 IEEE symposium on security and privacy, P177-187.

[12] Rob Pickering, Internet firewall tutorial, http://www.ipcortex.co.uk/wp/fw.rhtm, September 2005

[13] A.Rubin, D.Geer and M.Ranum. Web security sourcebook. Wiley Computer Publishing, 1997.

[14] Christoph L. Schuba. On the modeling, design, and implementation of firewall technology. Doctoral Dissertation, Purdue University, December 1997.

[15] L. Yuan, J. Mai, Z. Su, H. Chen, C-N. Chuah, and P. Mohapatra, FIREMAN: A Toolkit for Firewall Modeling and Analysis. IEEE Symposium on Security and Privacy, P199-213. May 2006. 


\section{Appendixes}

Appendix 1: Code for the example of multiple personal firewalls

The network architecture refers to Figure 4,1. The Complete Program for the example is following:

* The CONSTANT, the number may be much smaller than actual for simplicity*/

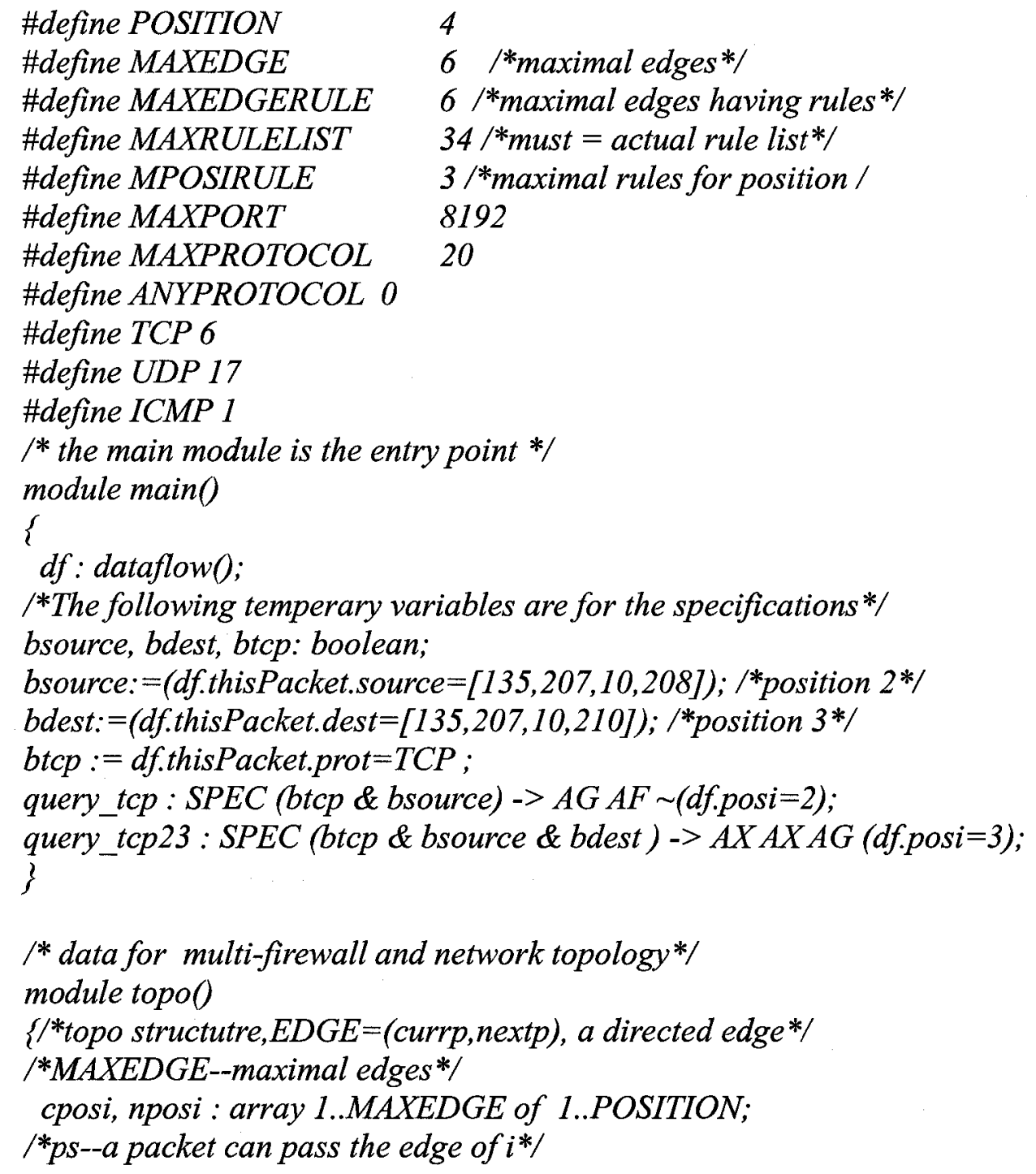




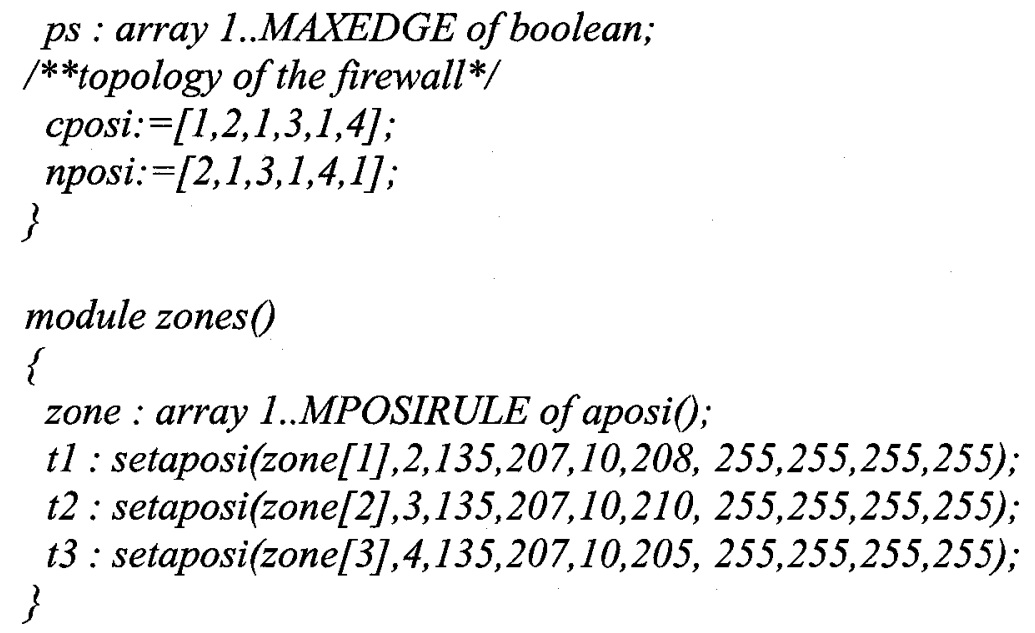

/*The following are relevant to specific firewalls*/ module rulesets()

t12: setarule(rules [2], 1,1, UDP, 0,0,0,0, 0,0,0,0, 135,207,10,208, 255,255,255,255, 53,53);

t13: setarule(rules [3], 1,1, UDP, 0,0,0,0, 0,0,0,0, 135,207,10,208, 255,255,255,255, $123,123)$;

t14 : setarule(rules[4], 1,1, ICMP, 0,0,0,0, 0,0,0,0, 135,207,10,208, 255,255,255,255, $8,8)$;

t15: setarule(rules [5], 1,1, ICMP, 0,0,0,0, 0,0,0,0, 135,207,10,208, 255,255,255,255, $0,0)$;

t16: setarule(rules[6], 1,1, ICMP, 0,0,0,0, 0,0,0,0, 135,207,10,208, 255,255,255,255, $113,113)$

$/$ rule 2 2-1*/

t21 : setarule(rules [7], 2,1, TCP, 135,207,10,208, 255,255,255,255, 0,0,0,0, 0,0,0,0, $0, M A X P O R T)$;

t22 : setarule(rules[8], 2,1, UDP, 135,207,10,208, 255,255,255,255, 0,0,0,0, 0,0,0,0, 53,53);

t23 : setarule(rules[9], 2,1, UDP, 135,207,10,208, 255,255,255,255, 0,0,0,0, 0,0,0,0, 123,123);

t24 : setarule(rules[10],2,1, ICMP, 135,207,10,208, 255,255,255,255, 0,0,0,0, 0,0,0,0, $8,8)$;

t25: setarule(rules[11],2,1, ICMP, 135,207,10,208, 255,255,255,255, 0,0,0,0, 0,0,0,0, 0,0 );

$/ *$ added rule denial $* * * * /$

t26: setarule(rules[12],2,0, ANYPROTOCOL, 0,0,0,0, 0,0,0,0,0,0,0,0,0,0,0,0,0, 
MAXPORT);

/*rule3--1-3*/

t31: setarule(rules[13],3,1, TCP, 0,0,0,0, 0,0,0,0,135,207,10,210, 255,255,255,255, $0, M A X P O R T)$;

t32: setarule(rules[14],3,1, UDP, 0,0,0,0, 0,0,0,0, 135,207,10,210, 255,255,255,255, 53,53);

t33 : setarule(rules[15],3,1, UDP, 0,0,0,0, 0,0,0,0, 135,207,10,210, 255,255,255,255, 123,123);

t34: setarule(rules[16],3,1, ICMP, 0,0,0,0, 0,0,0,0, 135,207,10,210, 255,255,255,255, $8,8)$;

t35: setarule(rules[17],3,1, ICMP, 0,0,0,0, 0,0,0,0, 135,207,10,210, 255,255,255,255, $0,0)$;

t36 : setarule(rules[18],3,1, ICMP, 0,0,0,0, 0,0,0,0, 135,207,10,210, 255,255,255,255, $113,113) ;$

/*rule4 3-1*/

t41: setarule(rules[19],4,1, TCP, 135,207,10,210, 255,255,255,255, 0,0,0,0, 0,0,0,0, $0, M A X P O R T)$;

t42: setarule(rules[20],4,1, UDP, 135,207,10,210, 255,255,255,255, 0,0,0,0, 0,0,0,0, 53,53);

t43 : setarule(rules[21],4,1, UDP, 135,207,10,210, 255,255,255,255, 0,0,0,0, 0,0,0,0, 123,123);

t44 : setarule(rules[22],4,1, ICMP, 135,207,10,210, 255,255,255,255, 0,0,0,0, 0,0,0,0, $8,8)$;

$t 45$ : setarule(rules[23],4,1, ICMP, 135,207,10,210,255,255,255,255, 0,0,0,0, 0,0,0,0, $0,0)$;

*rule5--1-4*/

t51: setarule(rules[24],5,1, TCP, 0,0,0,0, 0,0,0,0, 135,207,10,205, 255,255,255,255, $0, M A X P O R T)$;

t52 : setarule(rules[25],5,1, UDP, 0,0,0,0, 0,0,0,0, 135,207,10,205, 255,255,255,255, 53,53);

t53 : setarule(rules[26],5,1, UDP, 0,0,0,0, 0,0,0,0, 135,207,10,205, 255,255,255,255, 123,123);

t54 : setarule(rules[27],5,1, ICMP, 0,0,0,0, 0,0,0,0, 135,207,10,205, 255,255,255,255, $8,8)$;

t55 : setarule(rules[28],5,1, ICMP, 0,0,0,0, 0,0,0,0, 135,207,10,205, 255,255,255,255, $0,0)$;

t56 : setarule(rules[29],5,1, ICMP, 0,0,0,0, 0,0,0,0, 135,207,10,205, 255,255,255,255, $113,113)$;

/*rule6 4-1*/

t61 : setarule(rules[30],6,1, TCP, 135,207,10,205, 255,255,255,255, 0,0,0,0, 0,0,0,0, $0, M A X P O R T)$

t62: setarule(rules[31],6,1, UDP, 135,207,10,205, 255,255,255,255, 0,0,0,0, 0,0,0,0, 53,53);

t63 : setarule(rules[32],6,1, UDP, 135,207,10,205, 255,255,255,255, 0,0,0,0, 0,0,0,0, 123,123); 
t64 : setarule(rules[33],6,1, ICMP, 135,207,10,205, 255,255,255,255, 0,0,0,0, 0,0,0,0, $8,8)$;

t65 : setarule(rules[34],6,1, ICMP, 135,207,10,205, 255,255,255,255, 0,0,0,0, 0,0,0,0, $0,0)$; \}

The kernel code for the packet data flow is in Appendix 2. 
Appendix 2: the core code of our tool

When a spoofing attack is considered, the statement for init(posi) in module dataflow() should be removed since packets do not start from their actual source addresses. If routing information is not modelled, remove the statement $\{r t:$ routing(pack.dest); ) and its associates.

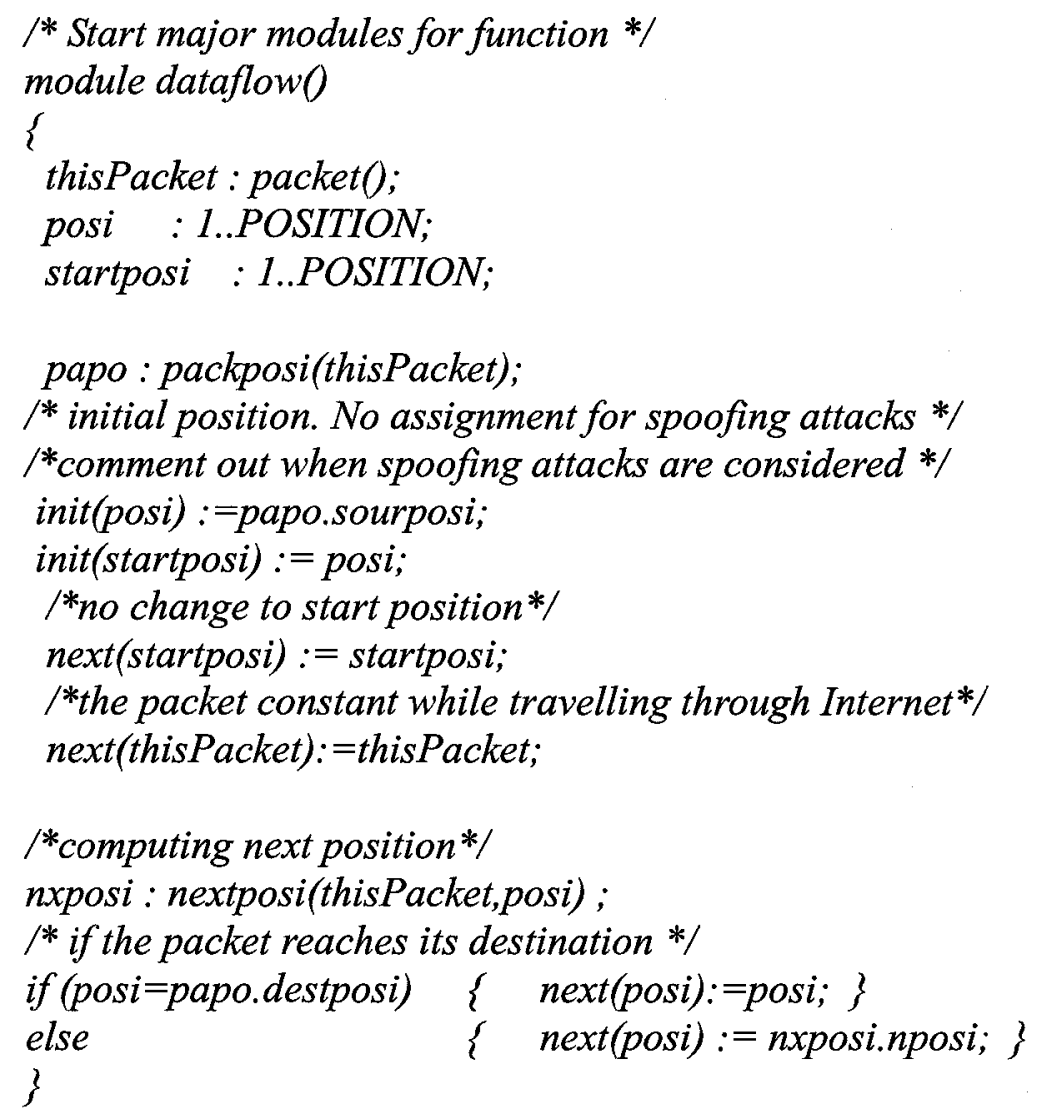

module nextposi(pack,currposi)

$\left\{/{ }^{*}\right.$ packet and current position : currposi*/

input pack : packet();

input currposi : 1..POSITION;

$/ *_{\text {next position*/ }}$

nposi : 1..POSITION;

$/$ topo structutre,Edge=(currposi,nextposi), a directed edge*/

wl : walls (pack);

$r t$ : routing(pack.dest);

tmp : array 1..MAXEDGE of boolean; /*temp variables*/

for $(i=1 ; i<=M A X E D G E ; i=i+1)$

$\operatorname{tmp}[i]:=(w l . t p . p s[i] \&$ rt.path [i] \& (wl.tp.cposi[i]=currposi) $) ;$ 
/* \& rt.path[i] true of tmp means a packet can pass $i$ interface into nextposi*/ if $(\mid$ tmp $)$

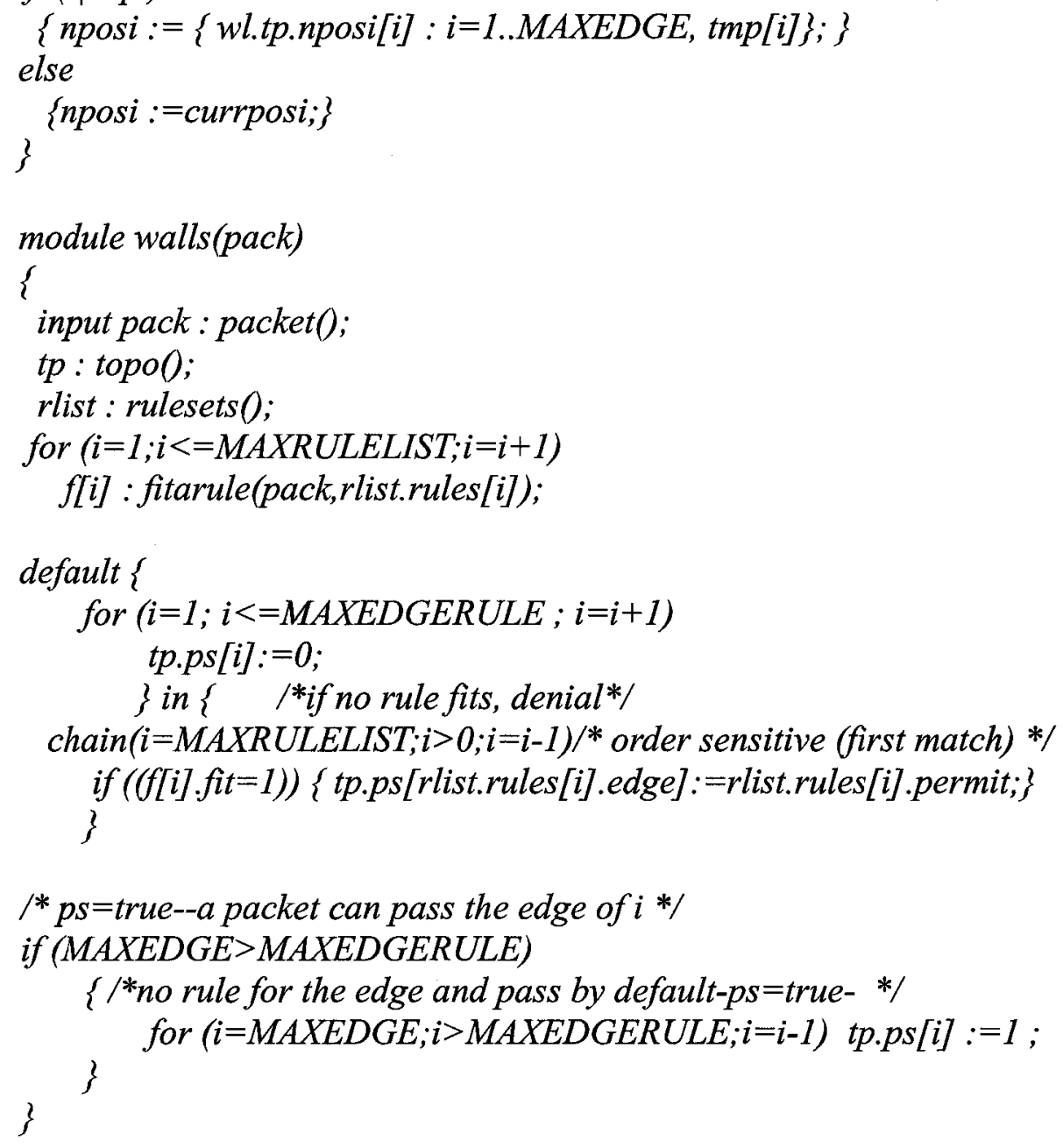




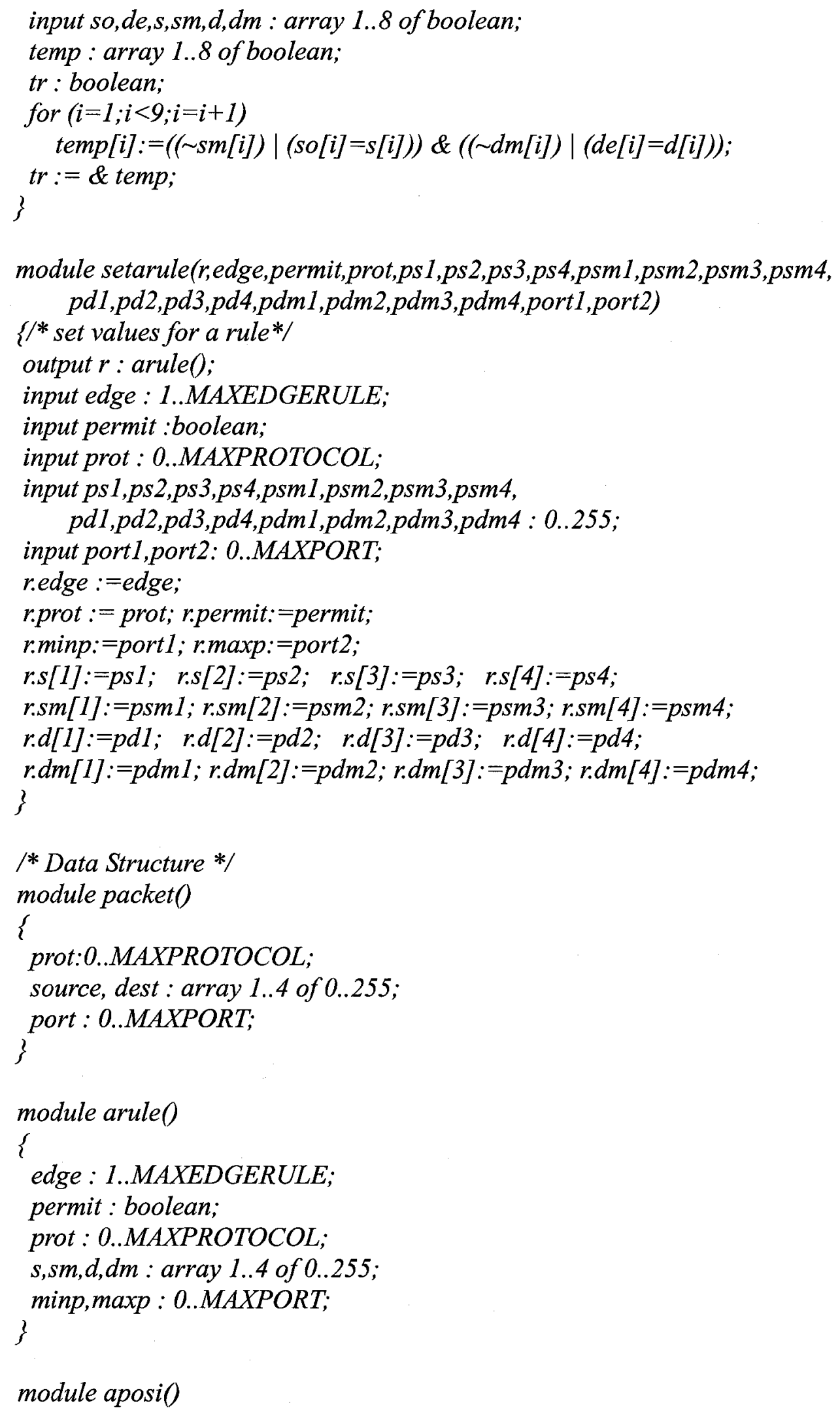

module aposi 0 


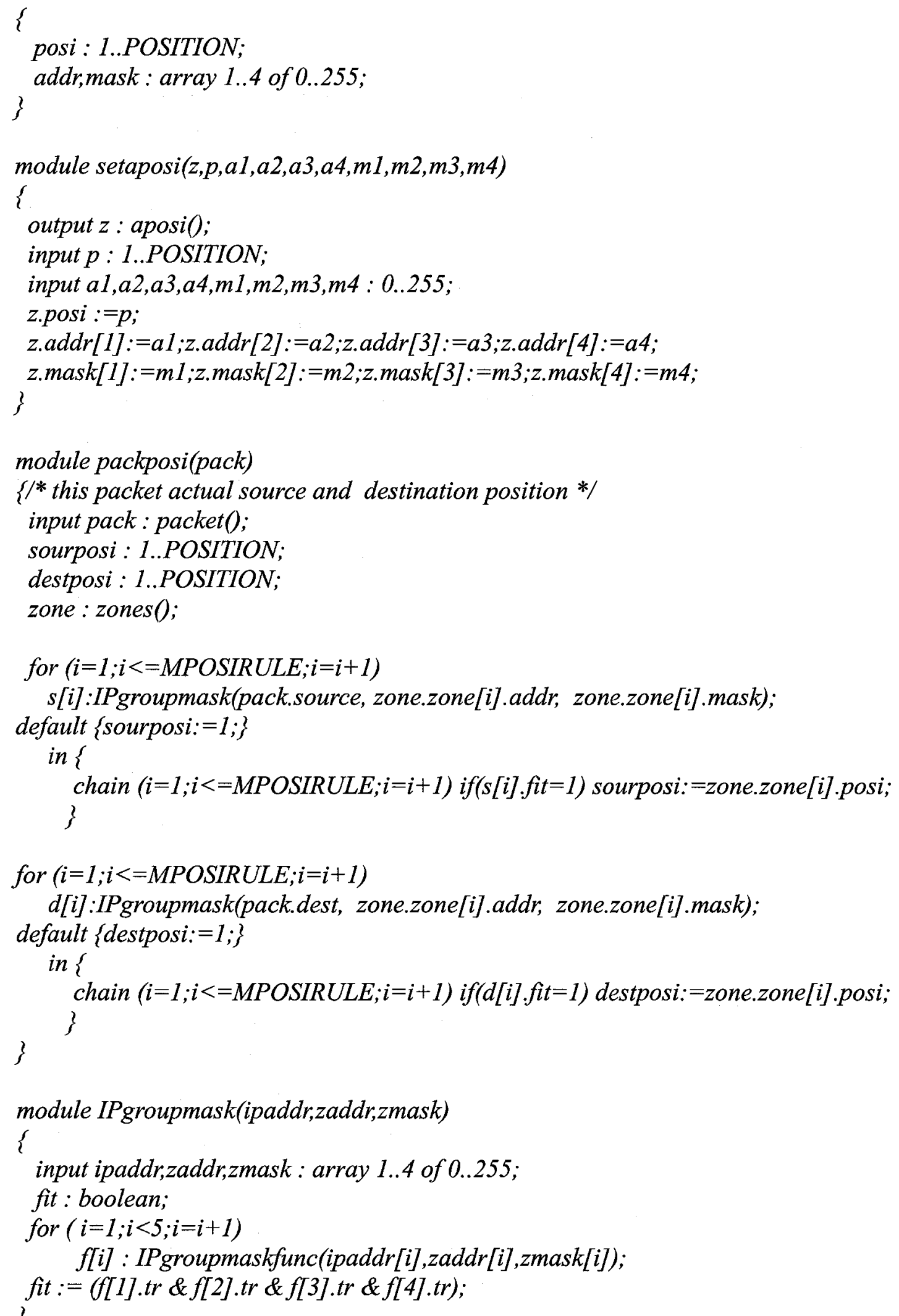




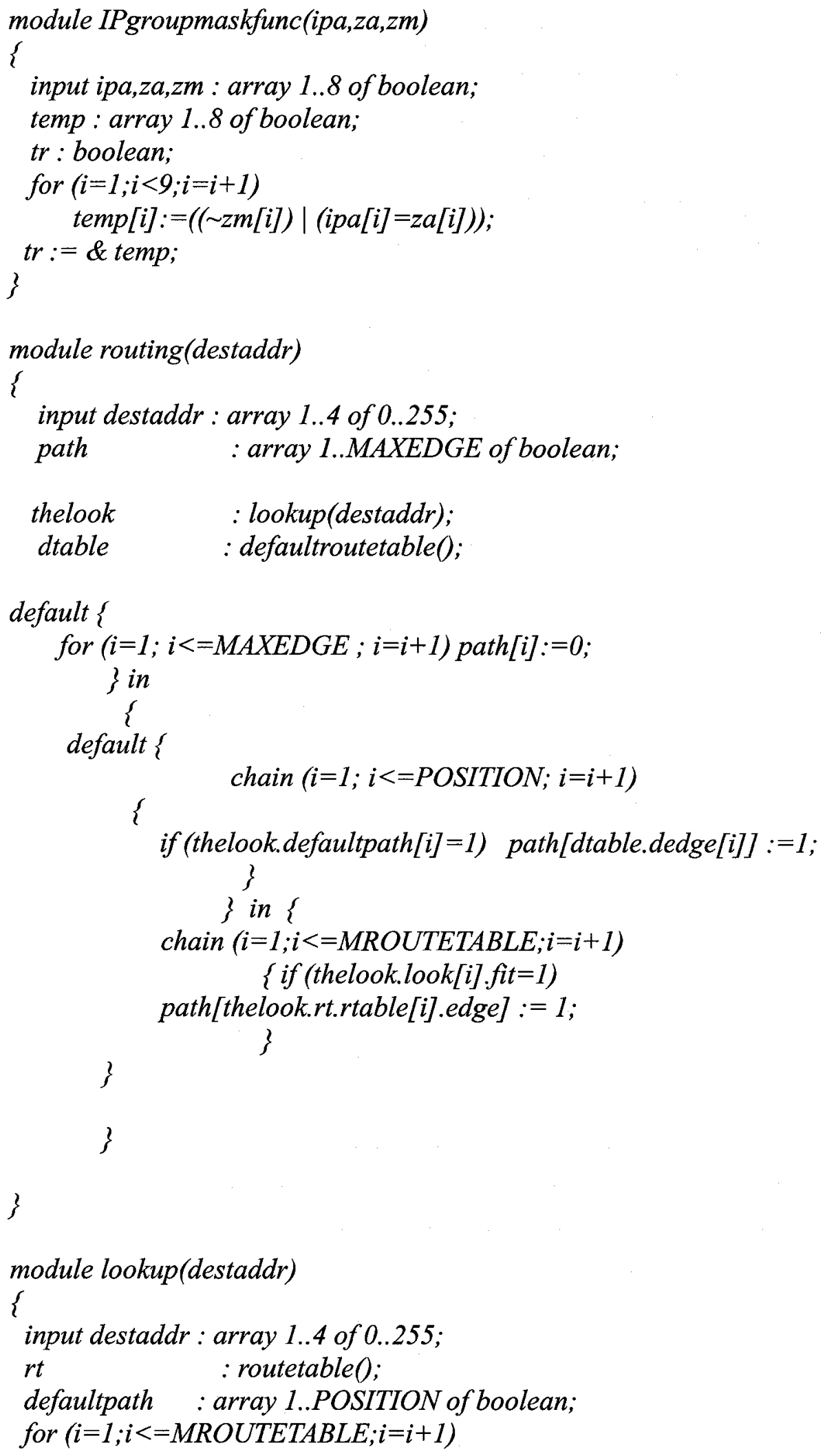


look[i]:IPgroupmask(destaddr, rt.rtable[i].addr, rt.rtable[i].mask);

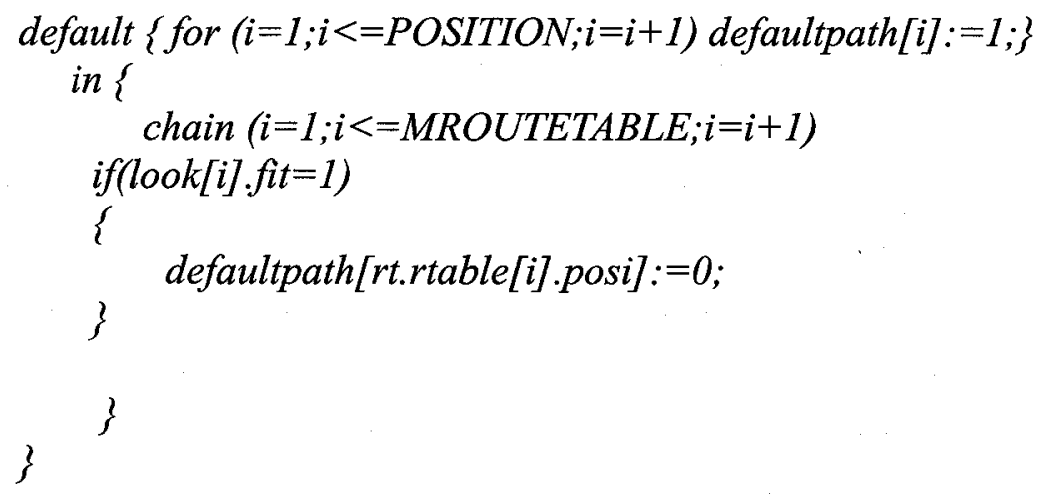

/* Ending major modules of implementation */ 
Appendix 3: Code for the example of multiple firewalls for an institution

The network architecture refers to Figure 6,1. The Complete Program for the example is following:

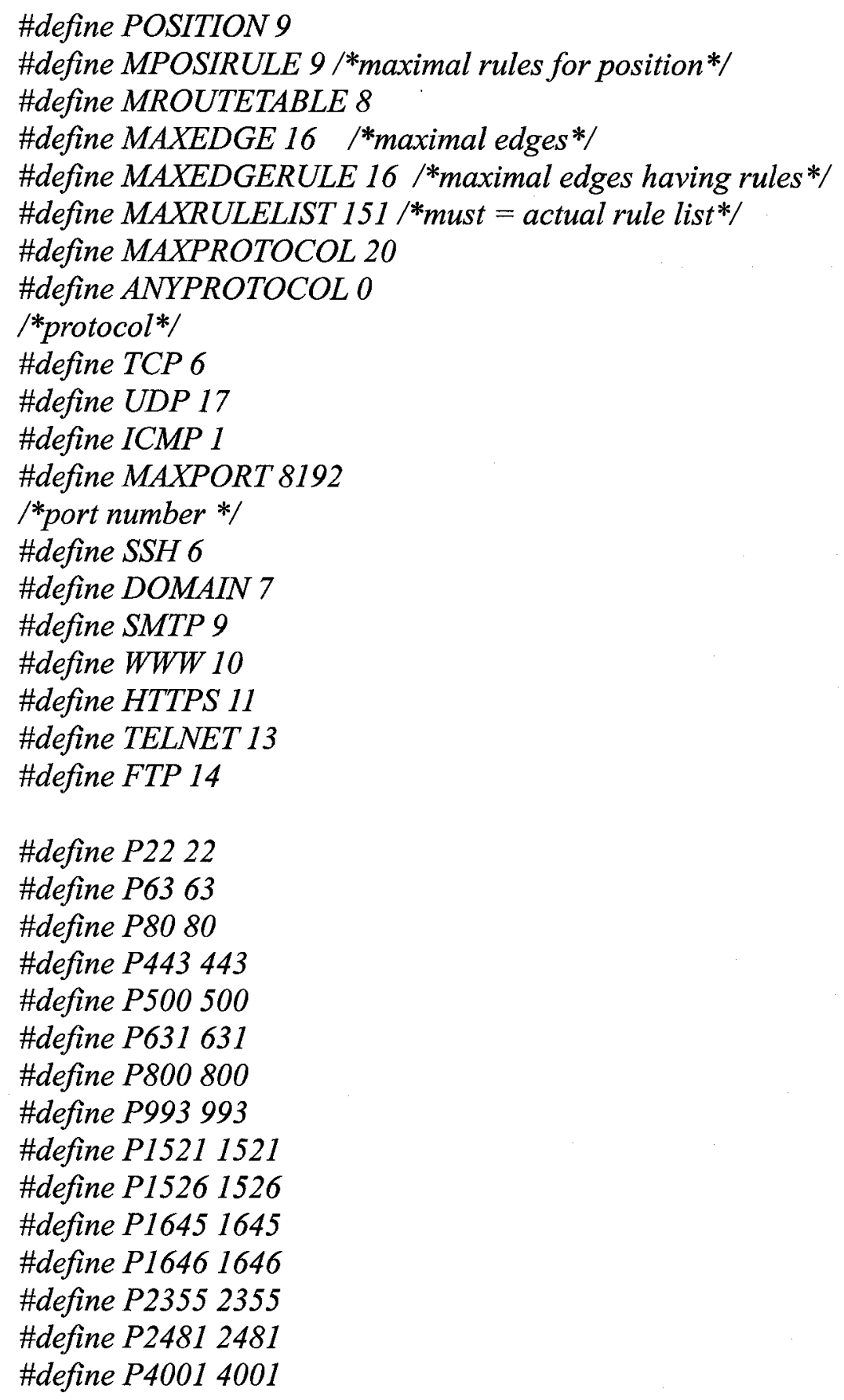


\#define P5001 5001

\#define P6881 6881

\#define P7001 7001

\#define P31950 7950

module main()

\{

df : dataflow();

/*The following are relevant to specific firewalls*/

/*The following temperary variables are for the specifications*/

/*protocol*/

btcp, budp, bicmp : boolean;

btcp $:=($ df.thisPacket.prot $=T C P)$;

budp $:=($ df.thisPacket.prot $=U D P)$;

bicmp: $=($ df.thisPacket.prot $=I C M P)$;

bsipdomain,bsip225,bsip5,bsip28 : boolean;

bsipdomain : $=($ df.thisPacket.source $[1]=234$

\& df.thisPacket.source[2]=217);

bsip225:= (bsipdomain \& (df.thisPacket.source [3]=225));

bsip5 := (bsipdomain \& (df.thisPacket.source [3]=5));

bsip28:=(bsipdomain \& (df.thisPacket.source [3]=28));

bdipdomain,bdip225,bdip5,bdip28 : boolean;

bdipdomain: $=($ df.thisPacket.dest $[1]=234$

$\&$ df.thisPacket.dest [2]=217);

bdip225:= (bdipdomain \& (df.thisPacket.dest [3]=225));

bdip5 := (bdipdomain \& (df.thisPacket.dest [3]=5));

bdip28:=(bdipdomain \& (df.thisPacket.dest [3]=28));

/* port number */

bpwww,bp22, bp631 : boolean;

bpwww :=(df.thisPacket.port $=W W W$ );

bp22 $:=($ df.thisPacket.port $=$ P22);

bp631 :=(df.thisPacket.port $=$ P631);

bdestlabawww : boolean;

bdestlabawww := bdip225\& df.thisPacket.dest[4]=13;

$/ *$ don't missing protocol $* /$

$/ *$ any can reach the $w w w$ server in lab $A * /$

brestrictedlab : boolean;

brestrictedlab $:=($ df.startposi $=6) \mid($ df.startposi=9) $\mid($ df.startposi $=3) \mid($ df.startposi $=2)$; 
query1 : SPEC (btcp \& bpwww \& ( (bsipdomain)) \& bdestlabawww) -> EF (df.posi $=2)$; query2 : SPEC (btcp \& bpwww \& ( brestrictedlab) \& bdestlabawww) -> EF ( (df.posi $=$ 2));

query3 : SPEC (btcp \& df.startposi=3 \& df.thisPacket.source [4]>48) ->AG (df.posi=3);

bdestlabb631 : boolean;

bdestlabb631:= bdip225\&df.thisPacket.dest [4]=41;

query4 : SPEC (btcp \& bp631 \& bdestlabb631 \& df.startposi=4) -> EF (df.posi=3);

query5 : SPEC (btcp \& bp631 \& bdestlabb631\&

$\sim((d f$. startposi=3) | (bsip5) )) -> AG (df.posi=3);

bdestcwww : boolean;

bdestcwww := bdip5 \& df.thisPacket.dest [4]=14;

query6 : SPEC (btcp \& bpwww \& ( (bsipdomain)) \& bdestcwww) -> EF (df.posi $=4)$;

bprintservice : boolean;

bprintservice $:=($ df.thisPacket.port $=$ P2355 $)$;

bdestcprn : boolean;

bdestcprn := bdip5 \& df.thisPacket.dest [4]=2;

query 7 : SPEC (bprintservice \& ( (bsipdomain) ) \& bdestcprn ) ->AG (df.posi $=4)$;

bdestdwww : boolean;

bdestdwww := bdip5 \& df.thisPacket.dest [4]=131;

bdestewww : boolean;

bdestewww := bdip5 \& df.thisPacket.dest [4]=133;

bsourf : boolean;

bsourf := bsip28\&df.thisPacket.source [4]=22;

query8 : SPEC (btcp \& bpwww \& (bsip5) \& bdestdwww) -> EF (df.posi = 5);

query9 : SPEC (btcp \& bpwww \& (bsourf) \& bdestewww) ->AF AG (df.posi =6);

query10 : SPEC (df.startposi=6 \& ( bdip5)) -> AG ((df.posi $=6))$;

\}

/* Beginning of data of multifirewall and network topology*/

module topo()

$\{/ *$ topo structutre,EDGE=(currp,nextp), a directed edge*/

/MAXEDGE--maximal edges*/

cposi, nposi : array 1..MAXEDGE of 1..POSITION;

/*ps--a packet can pass the edge of $i^{*} /$

ps : array 1..MAXEDGE of boolean;

**topology of the firewall*/ 


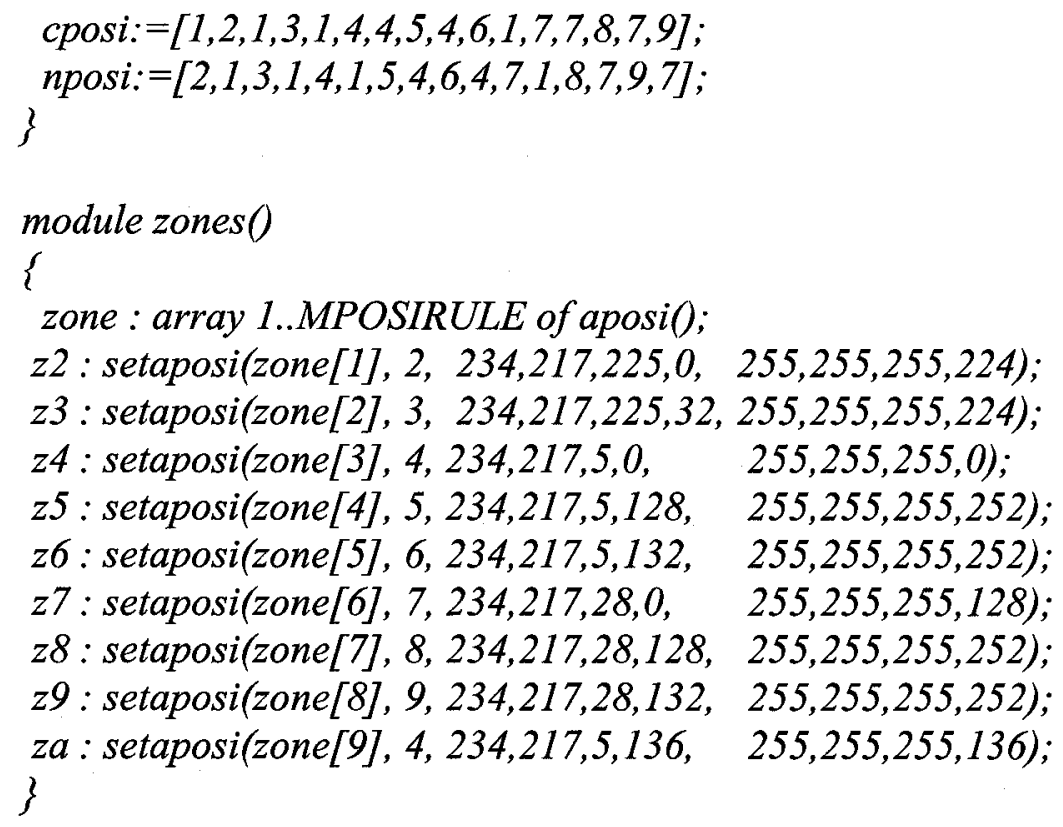

/*The following are relevant to specific firewalls*/ module rulesets()

\{ rules : array 1..MAXRULELIST of arule();

***set initial value*/

/ from position 1 to $2 *$ /

t11: setarule(rules [1], 1,1, ICMP, 0,0,0,0, 0,0,0,0,0,0,0,0,0,0,0,0, 0,MAXPORT); 
t12: setarule(rules[2],1,1, TCP,0,0,0,0,0,0,0,0, 234,217,225,13, 255,255,255,255, SSH, SSH);

t13 : setarule(rules[3],1,1, TCP,234,217,5,0, 255,255,255,0, 234,217,225,11, 255,255,255,255, P631, P631);

t14: setarule(rules [4],1,1, TCP,0,0,0,0, 0,0,0,0, 234,217,225,11, 255,255,255,255, DOMAIN, DOMAIN);

t15: setarule(rules[5],1,1, UDP,0,0,0,0, 0,0,0,0, 234,217,225,11, 255,255,255,255, DOMAIN, DOMAIN);

t16: setarule(rules[6],1,1, TCP,0,0,0,0,0,0,0,0, 234,217,225,11, 255,255,255,255, SMTP, SMTP);

t17 : setarule(rules[7],1,1, TCP,0,0,0,0,0,0,0,0, 234,217,225,11, 255,255,255,255, P993, P993);

t18: setarule(rules[8],1,1, TCP,0,0,0,0,0,0,0,0, 234,217,225,13, 255,255,255,255, WWW, $W W W)$;

t19: setarule(rules [9],1,1, TCP,0,0,0,0, 0,0,0,0, 234,217,225,13, 255,255,255,255, HTTPS, HTTPS);

t110 : setarule(rules[10],1,1, TCP,0,0,0,0,0,0,0,0, 234,217,225,13, 255,255,255,255, DOMAIN, DOMAIN);

t111 : setarule(rules[11],1,1, UDP,0,0,0,0, 0,0,0,0, 234,217,225,13, 255,255,255,255, DOMAIN, DOMAIN);

t112: setarule(rules[12],1,1, TCP,0,0,0,0,0,0,0,0, 234,217,225,20, 255,255,255,255, P6881, P6881);

t113: setarule(rules[13],1,1, TCP,0,0,0,0,0,0,0,0, 234,217,225,21, 255,255,255,255, P6881, P6881);

t114: setarule(rules [14],1,1, TCP,0,0,0,0,0,0,0,0, 234,217,225,22, 255,255,255,255, P6881, P6881);

t115: setarule(rules[15],1,1, TCP,0,0,0,0,0,0,0,0, 234,217,225,23, 255,255,255,255, P6881, P6881);

t116: setarule(rules[16],1,1, TCP,0,0,0,0, 0,0,0,0, 234,217,225,24, 255,255,255,255, P6881, P6881);

t117 : setarule(rules[17],1,1, TCP,0,0,0,0, 0,0,0,0, 234,217,225,25, 255,255,255,255, P6881, P6881);

t118: setarule(rules[18],1,1, TCP,0,0,0,0,0,0,0,0, 234,217,225,26, 255,255,255,255, P6881, P6881);

t119: setarule(rules[19],1,1, TCP,234,217,28,0, 255,255,255,255, 234,217,225,11, 255,255,255,255, P63, P63);

t120 : setarule(rules[20],1,1, TCP,0,0,0,0,0,0,0,0, 234,217,225,26, 255,255,255,255, P6881, P6881);

t121: setarule(rules[21],1,1, TCP,0,0,0,0,0,0,0,0, 234,217,225,31, 255,255,255,255, SSH, SSH);

t122: setarule(rules [22],1,1, TCP,0,0,0,0, 0,0,0,0, 0,0,0,0, 0,0,0,0, P500, P500);

/*from position 2 to $1 * /$

t223 : setarule(rules[23],2,1, TCP,234,217,225,11,255,255,255,255, 0,0,0,0, 0,0,0,0, SMTP, SMTP); 
t224: setarule(rules [24],2,0, TCP,0,0,0,0,0,0,0,0, 0,0,0,0, 0,0,0,0, SMTP, SMTP);

t225: setarule(rules[25],2,1, TCP,234,217,225,0, 255,255,255,240, 0,0,0,0,0,0,0,0, 0 , MAXPORT);

t226: setarule(rules[26],2,1, UDP,234,217,225,0, 255,255,255,240, 0,0,0,0, 0,0,0,0, 0 , MAXPORT);

t227: setarule(rules [27],2,1, ICMP,0,0,0,0, 0,0,0,0, 0,0,0,0, 0,0,0,0, 0, MAXPORT);

t228: setarule(rules [28],2,0, TCP,0,0,0,0,0,0,0,0,0,0,0,0,0,0,0,0, 0, MAXPORT);

t229: setarule(rules[29],2,0, UDP,0,0,0,0,0,0,0,0,0,0,0,0, 0,0,0,0, 0, MAXPORT);

/*from position 1 to 3 all $+30 * /$

t330: setarule(rules [30], 3,1, ICMP, 0,0,0,0, 0,0,0,0, 0,0,0,0, 0,0,0,0,0,MAXPORT);

t331: setarule(rules[31],3,1, TCP,0,0,0,0,0,0,0,0, 234,217,225,43, 255,255,255,255, SSH, SSH);

t332 : setarule(rules [32],3,1, TCP,234,217,5,0, 255,255,255,0, 234,217,225,41, $255,255,255,255, P 631, P 631)$;

t333: setarule(rules[33],3,1, TCP,0,0,0,0,0,0,0,0, 234,217,225,41, 255,255,255,255, DOMAIN, DOMAIN);

t334: setarule(rules [34],3,1, UDP,0,0,0,0,0,0,0,0, 234,217,225,41, 255,255,255,255, DOMAIN, DOMAIN);

t335: setarule(rules[35],3,1, TCP,0,0,0,0, 0,0,0,0, 234,217,225,41, 255,255,255,255, SMTP, SMTP);

t336 : setarule(rules[36],3,1, TCP,0,0,0,0,0,0,0,0, 234,217,225,41, 255,255,255,255, P993, P993);

t337 : setarule(rules [37],3,1, TCP,0,0,0,0, 0,0,0,0, 234,217,225,43, 255,255,255,255, $W W W, W W W)$;

t338: setarule(rules[38],3,1, TCP,0,0,0,0,0,0,0,0, 234,217,225,43, 255,255,255,255, HTTPS, HTTPS);

t339: setarule(rules [39],3,1, TCP,0,0,0,0, 0,0,0,0, 234,217,225,43, 255,255,255,255, DOMAIN, DOMAIN);

t340 : setarule(rules[40],3,1, UDP,0,0,0,0,0,0,0,0, 234,217,225,43, 255,255,255,255, DOMAIN, DOMAIN);

t341: setarule(rules[41],3,1, TCP,0,0,0,0, 0,0,0,0, 234,217,225,50, 255,255,255,255, P6881, P6881);

t342 : setarule(rules[42],3,1, TCP,0,0,0,0, 0,0,0,0, 234,217,225,51, 255,255,255,255, P6881, P6881);

t343 : setarule(rules [43],3,1, TCP,0,0,0,0, 0,0,0,0, 234,217,225,52, 255,255,255,255, P6881, P6881);

t344: setarule(rules[44],3,1, TCP,0,0,0,0,0,0,0,0, 234,217,225,53, 255,255,255,255, P6881, P6881);

t345: setarule(rules[45],3,1, TCP,0,0,0,0, 0,0,0,0, 234,217,225,54, 255,255,255,255, P6881, P6881);

t346: setarule(rules[46],3,1, TCP,0,0,0,0, 0,0,0,0, 234,217,225,55, 255,255,255,255, P6881, P6881);

t347 : setarule(rules[47],3,1, TCP,0,0,0,0, 0,0,0,0, 234,217,225,56, 255,255,255,255, P6881, P6881); 
t348: setarule(rules[48],3,1, TCP,234,217,28,0, 255,255,255,255, 234,217,225,41, $255,255,255,255, P 63, P 63)$;

t349: setarule(rules [49],3,1, TCP,0,0,0,0,0,0,0,0, 234,217,225,56, 255,255,255,255, P6881, P6881);

t350: setarule(rules [50],3,1, TCP,0,0,0,0,0,0,0,0, 234,217,225,31, 255,255,255,255, SSH, SSH);

t351: setarule(rules[51],3,1, TCP,0,0,0,0,0,0,0,0,0,0,0,0,0,0,0,0, P500, P500);

/*edge 4 = from position 3 to $1 * /$

t452: setarule(rules[52],4,1, TCP,234,217,225,41,255,255,255,255, 0,0,0,0, 0,0,0,0, SMTP, SMTP);

t453 : setarule(rules [53],4,0, TCP,0,0,0,0, 0,0,0,0, 0,0,0,0, 0,0,0,0, SMTP, SMTP);

t454: setarule(rules[54],4,1, TCP,234,217,225,32, 255,255,255,240, 0,0,0,0,0,0,0,0, 0 , MAXPORT);

t455: setarule(rules[55],4,1, UDP,234,217,225,32, 255,255,255,240, 0,0,0,0,0,0,0,0, 0 , $M A X P O R T)$;

t456: setarule(rules[56],4,1, ICMP,0,0,0,0,0,0,0,0,0,0,0,0,0,0,0,0, 0, MAXPORT);

t457 : setarule(rules [57],4,0, TCP,0,0,0,0,0,0,0,0,0,0,0,0,0,0,0,0, 0, MAXPORT);

/No UDP*/

*edge $5=$ from position 1 to $4 * /$

/* Prevent Spoofing attacking */

t558: setarule(rules [58],5,0, ANYPROTOCOL,234,217,5,0, 255,255,255,0, 0,0,0,0, $0,0,0,0,0, M A X P O R T)$;

$/ *$ let Cisco access

t558a: setarule(rules[58],5,1, TCP, 0,0,0,0,0,0,0,0, 234,217,5,50, 255,255,255,255, SMTP, SMTP);

t559: setarule(rules[59],5,0, ANYPROTOCOL,64,102,51,20,255,255, 255,255, 0,0,0,0, $*$ / $0,0,0,0,0, M A X P O R T)$;

t559: setarule(rules[59],5,1, TCP, 0,0,0,0, 0,0,0,0, 234,217,5,50, 255,255,255,255, SMTP, SMTP);

t560 : setarule(rules[60],5,1, TCP, 0,0,0,0, 0,0,0,0, 234,217,5,50, 255,255,255,255, SMTP, SMTP);

t561 : setarule(rules[61],5,1, TCP, 0,0,0,0, 0,0,0,0, 234,217,5,50, 255,255,255,255, P993, P993);

t562 : setarule(rules[62],5,1, TCP, 0,0,0,0,0,0,0,0, 234,217,5,50, 255,255,255,255, P31950, P31950);

t563 : setarule(rules[63],5,1, UDP, 0,0,0,0,0,0,0,0, 234,217,5,50, 255,255,255,255, P31950, P31950);

t564: setarule(rules [64],5,1, TCP, 0,0,0,0,0,0,0,0, 234,217,5,11, 255,255,255,255, DOMAIN, DOMAIN);

t565: setarule(rules[65],5,1, UDP, 0,0,0,0, 0,0,0,0, 234,217,5,11, 255,255,255,255, DOMAIN, DOMAIN); 
t566 : setarule(rules[66],5,1, TCP, 0,0,0,0,0,0,0,0,234,217,5,11, 255,255,255,255, P80, P80);

t567 : setarule(rules[67],5,1, TCP, 0,0,0,0,0,0,0,0, 234,217,5,11, 255,255,255,255, P443, P443);

t568: setarule(rules[68],5,1, TCP, 0,0,0,0,0,0,0,0, 234,217,5,9, 255,255,255,255, P80, P80);

t569: setarule(rules[69],5,1, TCP, 0,0,0,0,0,0,0,0, 234,217,5,9, 255,255,255,255, P443, P443);

t570: setarule(rules[70],5,1, TCP, 234,217,0,0, 255,255,0,0, 234,217,5,2, $255,255,255,255, P 2355, P 2355)$;

t571: setarule(rules[71],5,1, UDP, 234,217,0,0, 255,255,0,0, 234,217,5,2, $255,255,255,255, P 2355, P 2355)$;

t572: setarule(rules[72],5,1, TCP, 0,0,0,0,0,0,0,0, 234,217,5,49, 255,255,255,255, DOMAIN, DOMAIN);

t573 : setarule(rules[73],5,1, UDP, 0,0,0,0, 0,0,0,0, 234,217,5,49, 255,255,255,255, DOMAIN, DOMAIN);

t574 : setarule(rules[74],5,1, TCP, 0,0,0,0,0,0,0,0, 234,217,5,13, 255,255,255,255, TELNET, TELNET);

t575: setarule(rules[75],5,1, TCP, 0,0,0,0, 0,0,0,0, 234,217,5,13, 255,255,255,255, FTP, FTP);

t576: setarule(rules[76],5,1, TCP, 0,0,0,0,0,0,0,0, 234,217,5,13, 255,255,255,255, P22, P22);

t577 : setarule(rules [77],5,1, TCP, 0,0,0,0,0,0,0,0, 234,217,5,13, 255,255,255,255, P1645, P1645);

t578: setarule(rules[78],5,1, TCP, 0,0,0,0,0,0,0,0, 234,217,5,13, 255,255,255,255, P1646, P1646);

t579: setarule(rules[79],5,1, TCP, 0,0,0,0, 0,0,0,0, 234,217,5,120, 255,255,255,255, $W W W, W W W)$;

t580: setarule(rules[80],5,1, TCP, 0,0,0,0, 0,0,0,0, 234,217,5,120, 255,255,255,255, P800, P800);

t581: setarule(rules[81],5,1, TCP, 0,0,0,0,0,0,0,0, 234,217,5,14, 255,255,255,255, WWW, $W W W)$;

t582: setarule(rules[82],5,1, TCP, 0,0,0,0, 0,0,0,0, 234,217,5,14, 255,255,255,255, P443,P443);

t583 : setarule(rules[83],5,1, TCP, 0,0,0,0, 0,0,0,0, 234,217,5,56, 255,255,255,255, P22, P22);

t584: setarule(rules[84],5,1, TCP, 0,0,0,0,0,0,0,0, 234,217,5,15, 255,255,255,255, P22, P22);

t585: setarule(rules[85],5,1, TCP, 0,0,0,0,0,0,0,0, 234,217,5,63, 255,255,255,255, P22, P22);

$/ *$ goto the labs behind school. Adaption from real*/

t586: setarule(rules[86],5,1, ANYPROTOCOL, 0,0,0,0, 0,0,0,0, 234,217,5,128, $255,255,255,248,0, M A X P O R T)$;

t587 : setarule(rules [87],5,1, TCP, 0,0,0,0, 0,0,0,0, 234,217,5,24, 255,255,255,255, P22, 
P22);

t588: setarule(rules [88],5,1, TCP, 0,0,0,0,0,0,0,0,234,217,5,24, 255,255,255,255, WWW, $W W W)$;

t589: setarule(rules[89],5,1, TCP, 0,0,0,0, 0,0,0,0, 234,217,5,24, 255,255,255,255, P993, P993);

t590: setarule(rules[90],5,1, TCP, 0,0,0,0,0,0,0,0, 234,217,5,24, 255,255,255,255, P443, P443);

t591: setarule(rules[91],5,1, TCP, 0,0,0,0,0,0,0,0, 234,217,5,52, 255,255,255,255, P4001, P4001);

t592: setarule(rules [92],5,1, TCP, 0,0,0,0, 0,0,0,0, 234,217,5,52, 255,255,255,255, P5001, P5001);

/*edge 6 = from position 4 to $1 * /$

t693: setarule(rules[93],6,1, ANYPROTOCOL, 234,217,5,0, 255,255,255,0, 0,0,0,0, $0,0,0,0,0, M A X P O R T)$;

t694: setarule(rules[94],6,0, ANYPROTOCOL, 0,0,0,0, 0,0,0,0, 0,0,0,0,0,0,0,0, 0 , $M A X P O R T)$;

$/ *$ edge $7=$ from position 4 to $5 * /$

t795: setarule(rules[95],7,1, TCP, 0,0,0,0, 0,0,0,0, 234,217,5,131, 255,255,255,255, P22, P22);

t796 : setarule(rules[96],7,1, TCP, 0,0,0,0, 0,0,0,0, 234,217,5,131, 255,255,255,255, $W W W, W W W$;

t797: setarule(rules[97],7,1, TCP, 0,0,0,0, 0,0,0,0, 234,217,5,131, 255,255,255,255, P1521, P1521);

t798: setarule(rules[98],7,1, TCP, 0,0,0,0, 0,0,0,0, 234,217,5,131, 255,255,255,255, P1526, P1526);

t799: setarule(rules[99],7,1, TCP, 0,0,0,0, 0,0,0,0, 234,217,5,131, 255,255,255,255, P2481, P2481);

t700: setarule(rules[100],7,1, TCP, 0,0,0,0,0,0,0,0,234,217,5,130, 255,255,255,255, P22, P22);

/*edge 8 = from position 5 to $4 * /$

t801 : setarule(rules[101],8, 1, ANYPROTOCOL, 0,0,0,0,0,0,0,0, 0,0,0,0, 0,0,0,0, 0 , $M A X P O R T)$;

$/ *$ edge $9=$ from position 4 to $6 * *$ only from the school*/

t902 : setarule(rules[102],9,1, TCP, 234,217,5,0, 255,255,255,0, 234,217,5,133, $255,255,255,255, P 22, P 22)$;

t903 : setarule(rules[103],9,1, TCP, 234,217,5,0, 255,255,255,0, 234,217,5,133, $255,255,255,255, W W W, W W W)$;

t904 : setarule(rules[104],9,1, TCP, 234,217,5,0, 255,255,255,0, 234,217,5,133, $255,255,255,255, P 1521, P 1521)$;

*edge $10=$ from position 6 to $4 * /$ 
t105: setarule(rules [105],10,1, ANYPROTOCOL, 0,0,0,0,0,0,0,0, 234,217,5,0, 255,255,255,0, 0, MAXPORT);

$/ *$ edge $11=$ from position 1 to $7 * /$

* Prevent Spoofing attacking */

/*t558: setarule(rules [**],11,0, ANYPROTOCOL,234,217,28,0, 255,255,255,0, 0,0,0,0, $0,0,0,0,0, M A X P O R T) ; *$ /

* let Cisco access */

/*559 : setarule(rules[**],11,0, ANYPROTOCOL,64,102,51,20,255,255,255,255, 0,0,0,0, $0,0,0,0,0, M A X P O R T) ; * /$

t1106: setarule(rules[106],11,1, TCP, 0,0,0,0, 0,0,0,0, 234,217,28,50, 255,255,255,255, SMTP, SMTP);

t1107: setarule(rules[107],11,1, TCP, 0,0,0,0,0,0,0,0, 234,217,28,50, 255,255,255,255, P993, P993);

t1108: setarule(rules[108],11,1, TCP, 0,0,0,0, 0,0,0,0, 234,217,28,50, 255,255,255,255, P31950, P31950);

t1109: setarule(rules[109],11,1, UDP, 0,0,0,0, 0,0,0,0, 234,217,28,50, 255,255,255,255, P31950, P31950);

t1110 : setarule(rules[110],11,1, TCP, 0,0,0,0, 0,0,0,0, 234,217,28,11, 255,255,255,255, DOMAIN, DOMAIN);

t1111 : setarule(rules[111],11,1, UDP, 0,0,0,0, 0,0,0,0, 234,217,28,11, 255,255,255,255, DOMAIN, DOMAIN);

t1112 : setarule(rules[112],11,1, TCP, 0,0,0,0, 0,0,0,0, 234,217,28,11, 255,255,255,255, P80, P80);

t1113 : setarule(rules [113],11,1, TCP, 0,0,0,0,0,0,0,0, 234,217,28,11, 255,255,255,255, P443, P443);

t1114: setarule(rules[114],11,1, TCP, 0,0,0,0, 0,0,0,0, 234,217,28,9, 255,255,255,255, P80, P80);

t1115: setarule(rules[115],11,1, TCP, 0,0,0,0, 0,0,0,0, 234,217,28,9, 255,255,255,255, P443, P443);

t1116 : setarule(rules[116],11,1, TCP, 234,217,0,0, 255,255,0,0, 234,217,28,2, $255,255,255,255, P 2355, P 2355)$;

t1117 : setarule(rules[117],11,1, UDP, 234,217,0,0, 255,255,0,0, 234,217,28,2, 255,255,255,255, P2355, P2355);

t1118: setarule(rules[118],11,1, TCP, 0,0,0,0, 0,0,0,0, 234,217,28,49, 255,255,255,255, DOMAIN, DOMAIN);

t1119: setarule(rules[119],11,1, UDP, 0,0,0,0, 0,0,0,0, 234,217,28,49, 255,255,255,255, DOMAIN, DOMAIN);

t1120: setarule(rules[120],11,1, TCP, 0,0,0,0, 0,0,0,0, 234,217,28,13, 255,255,255,255, TELNET, TELNET);

t1121 : setarule(rules[121],11,1, TCP, 0,0,0,0, 0,0,0,0, 234,217,28,13, 255,255,255,255, FTP, FTP);

t1122: setarule(rules[122],11,1, TCP, 0,0,0,0, 0,0,0,0, 234,217,28,13, 255,255,255,255, P22, P22);

t1123: setarule(rules[123],11,1, TCP, 0,0,0,0,0,0,0,0, 234,217,28,13, 255,255,255,255, 
P1645, P1645);

t1124: setarule(rules[124],11,1, TCP, 0,0,0,0,0,0,0,0, 234,217,28,13, 255,255,255,255, P1646, P1646);

t1125: setarule(rules[125],11,1, TCP, 0,0,0,0, 0,0,0,0, 234,217,28,120, 255,255,255,255, $W W W, W W W$;

t1126: setarule(rules[126],11,1, TCP, 0,0,0,0,0,0,0,0, 234,217,28,120, 255,255,255,255, P800, P800);

t1127: setarule(rules[127],11,1, TCP, 0,0,0,0,0,0,0,0, 234,217,28,14, 255,255,255,255, $W W W, W W W$;

t1128: setarule(rules[128],11,1, TCP, 0,0,0,0, 0,0,0,0, 234,217,28,14, 255,255,255,255, P443,P443);

t1129: setarule(rules[129],11,1, TCP, 0,0,0,0, 0,0,0,0, 234,217,28,56, 255,255,255,255, P22, P22);

t1130: setarule(rules[130],11,1, TCP, 0,0,0,0,0,0,0,0, 234,217,28,15, 255, 255,255,255, P22, P22);

t1131: setarule(rules[131],11,1, TCP, 0,0,0,0,0,0,0,0, 234,217,28,63, 255,255,255,255, P22, P22);

/* goto the labs behind school. Adaption from real*/

t1132 : setarule(rules[132],11,1, ANYPROTOCOL, 0,0,0,0, 0,0,0,0, 234,217,28,128, 255,255,255,248, 0, MAXPORT);

t1133 : setarule(rules[133],11,1, TCP, 0,0,0,0, 0,0,0,0, 234,217,28,24, 255,255,255,255, P22, P22);

t1134: setarule(rules[134],11,1, TCP, 0,0,0,0,0,0,0,0, 234,217,28,24, 255,255,255,255, $W W W, W W W)$

t1135: setarule(rules[135],11,1, TCP, 0,0,0,0,0,0,0,0, 234,217,28,24, 255,255,255,255, P993, P993);

t1136: setarule(rules[136],11,1, TCP, 0,0,0,0,0,0,0,0, 234,217,28,24, 255,255,255,255, P443, P443);

t1137: setarule(rules[137],11,1, TCP, 0,0,0,0,0,0,0,0, 234,217,28,52, 255,255,255,255, P4001, P4001);

t1138: setarule(rules[138],11,1, TCP, 0,0,0,0,0,0,0,0, 234,217,28,52, 255,255,255,255, P5001, P5001);

/*edge 12 = from position 7 to $1 * /$

t1239: setarule(rules[139],12,1, ANYPROTOCOL, 234,217,28,0, 255,255,255,0, 0,0,0,0, $0,0,0,0,0, M A X P O R T)$;

t1240: setarule(rules[140],12,0, ANYPROTOCOL, 0,0,0,0, 0,0,0,0, 0,0,0,0, 0,0,0,0, 0 , MAXPORT);

$/$ edge $13=$ from position 7 to $8 * /$

t1341: setarule(rules[141],13,1, TCP, 0,0,0,0, 0,0,0,0, 234,217,28,131, 255,255,255,255, P22, P22);

t1342: setarule(rules[142],13,1, TCP, 0,0,0,0,0,0,0,0, 234,217,28,131, 255,255,255,255, $W W W, W W W)$; 
t1343 : setarule(rules[143],13,1, TCP, 0,0,0,0, 0,0,0,0, 234,217,28,131, 255,255,255,255, P1521, P1521);

t1344: setarule(rules[144],13,1, TCP, 0,0,0,0, 0,0,0,0, 234,217,28,131, 255,255,255,255, P1526, P1526);

t1345: setarule(rules[145],13,1, TCP, 0,0,0,0, 0,0,0,0, 234,217,28,131, 255,255,255,255, P2481, P2481);

t1346: setarule(rules[146],13,1, TCP, 0,0,0,0, 0,0,0,0, 234,217,28,130, 255,255,255,255, P22, P22);

$/ *$ edge $14=$ from position 8 to $7 * /$

t1447 : setarule(rules[147],14,1, ANYPROTOCOL, 0,0,0,0, 0,0,0,0, 0,0,0,0, 0,0,0,0, 0 , $M A X P O R T)$;

$/$ edge $15=$ from position 7 to $9 * *$ only from the school*/

t1548: setarule(rules[148],15,1, TCP, 234,217,28,0, 255,255,255,0, 234,217,28,131, $255,255,255,255, P 22, P 22)$;

t1549 : setarule(rules[149],15,1, TCP, 234,217,28,0, 255,255,255,0, 234,217,28,131, $255,255,255,255, W W W, W W W)$;

t1550 : setarule(rules[150],15,1, TCP, 234,217,28,0, 255,255,255,0, 234,217,28,131, 255,255,255,255, P1521, P1521);

/*edge $16=$ from position 6 to 4 */

t1651: setarule(rules[151],16,1, ANYPROTOCOL, 0,0,0,0, 0,0,0,0, 234,217,28,0, 255,255,0,0, 0, MAXPORT); 